\title{
Kathodale transkranielle Stromstimulation des visuellen Kortex als Verfahren zur prophylaktischen Behandlung der Migräne
}

\author{
INAUGURAL-DISSERTATION \\ zur Erlangung des Doktorgrades \\ der Medizinischen Fakultät der \\ Georg-August-Universität zu Göttingen
}

vorgelegt von

Naomi Mara Kriener

aus

Wuppertal

Göttingen 2020 
Dekan:

Referentin:

Ko-Referent:

Drittreferentin:
Prof. Dr. Wolfgang Brück

Prof. Dr. Andrea Antal

PD Dr. Joachim Erlenwein

Prof. Dr. Margarete Schön

Datum der mündlichen Prüfung: 24.03.2021 
Hiermit erkläre ich, die Dissertation mit dem Titel „Kathodale transkranielle Stromstimulation des visuellen Kortex als Verfahren zur prophylaktischen Behandlung der Migräne“ eigenständig angefertigt und keine anderen als die von mir angegebenen Quellen und Hilfsmittel verwendet zu haben.

Göttingen, den 09.12.2019 
Die Daten auf denen die vorliegende Arbeit basiert wurden teilweise publiziert:

Antal A, Kriener N, Lang N, Boros K, Paulus W (2011): Cathodal transcranial direct current stimulation of the visual cortex in the prophylactic treatment of migraine. Cephalalgia $\underline{31}$, $820-828$ 


\section{Inhaltsverzeichnis}

Abbildungsverzeichnis ......................................................................... III

Tabellenverzeichnis ..................................................................................

Abkürzungsverzeichnis ............................................................................ V

1 Einleitung und Zielsetzung ................................................................ 1

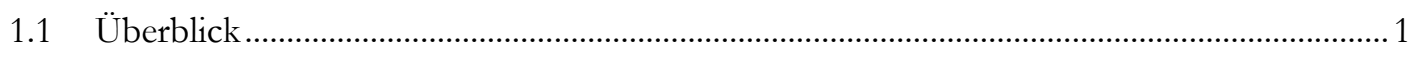

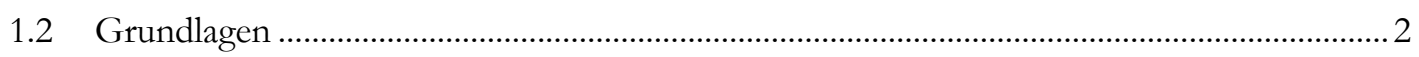

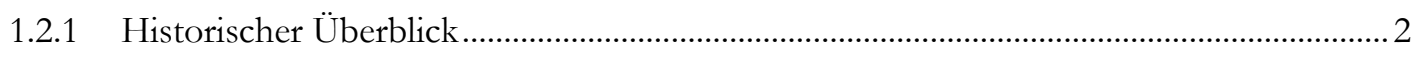

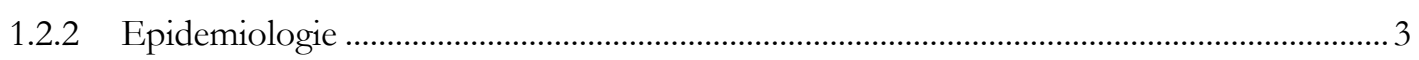

1.2.3 Klassifikation, Klinik und Diagnostik der Migräne ............................................................ 3

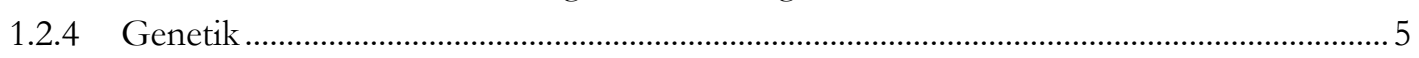

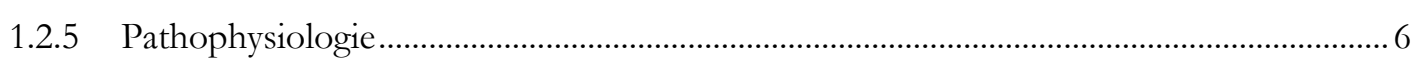

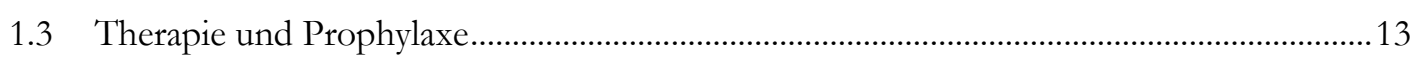

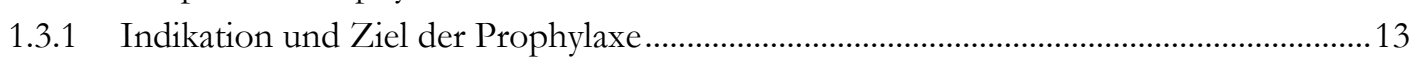

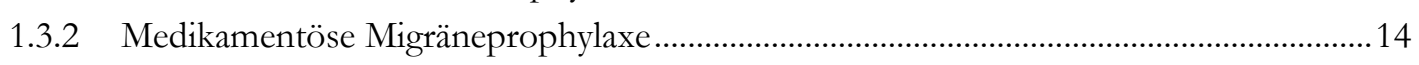

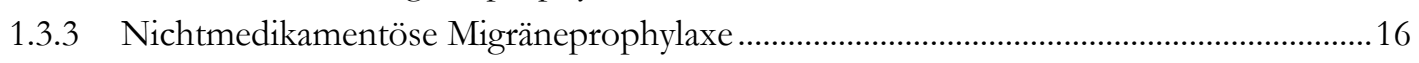

1.4 Neuromodulierende Verfahren zur Migränetherapie..........................................................16

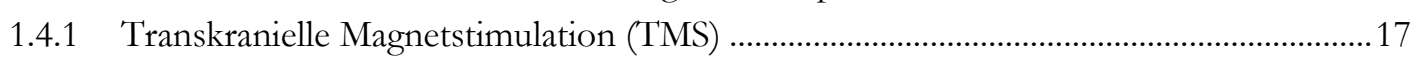

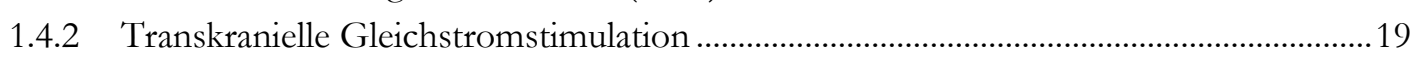

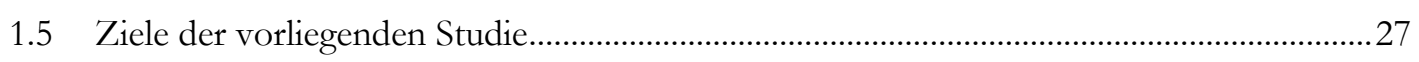

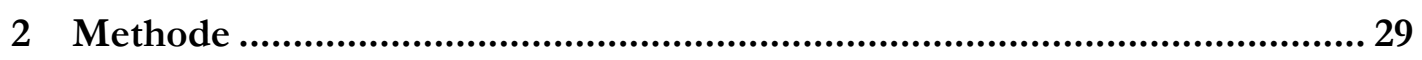

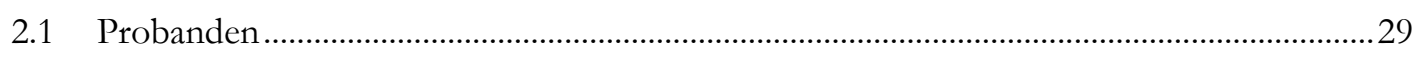

2.2 Studiendesign ..............................................................................................................

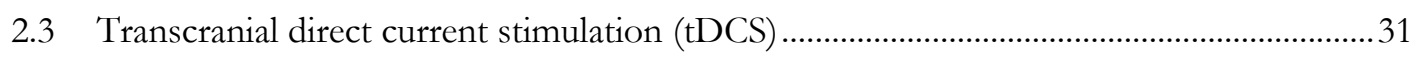

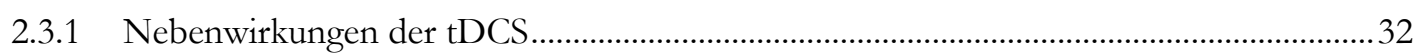

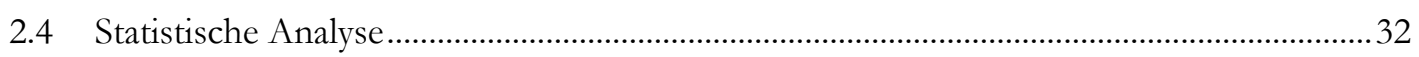

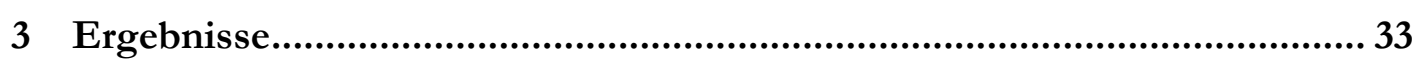

3.1 Frequenz der Migräneattacken....................................................................................... 33

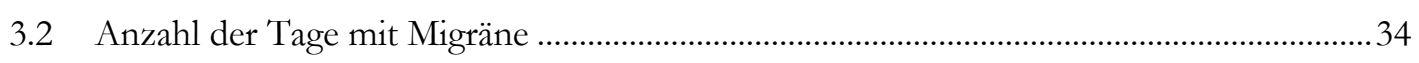

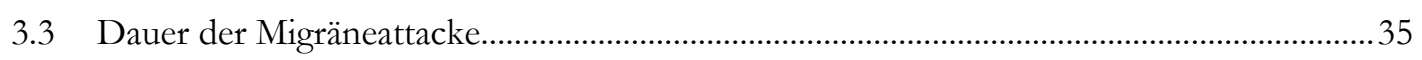

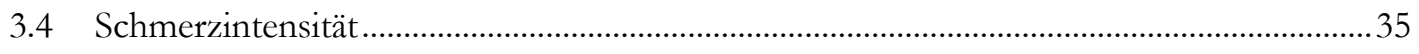

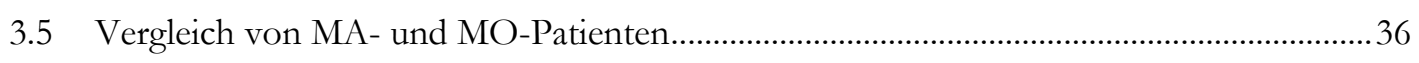

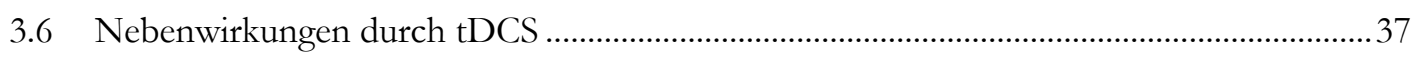

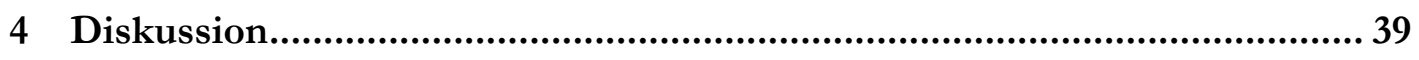

4.1 Vergleich mit Studien zu tDCS bei Migräne........................................................................

4.2 Vergleich mit Studien zu TMS bei Migräne....................................................................... 41 


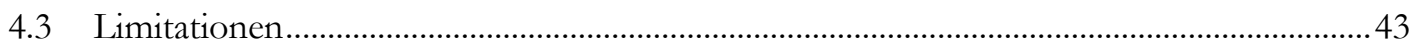

4.4 Nebenwirkungen und Patientensicherheit .........................................................................4

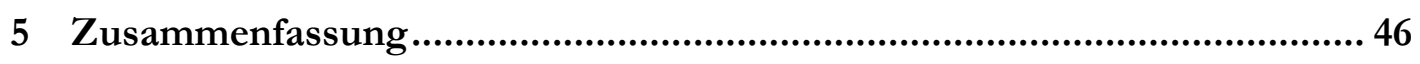

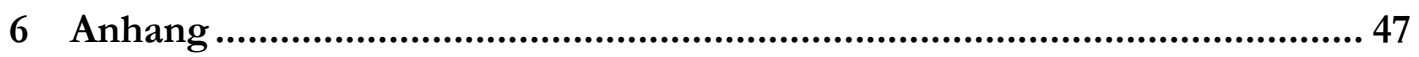

6.1 Verwendete Fragebögen .................................................................................................

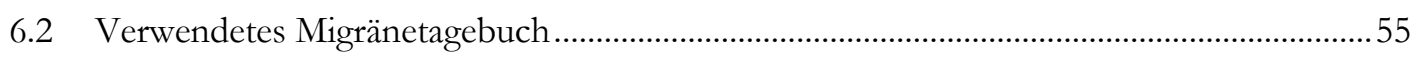

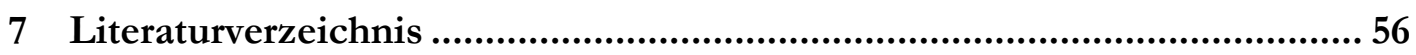




\section{Abbildungsverzeichnis}

Abbildung 1: visuelle Rindenfelder.....

Abbildung 2: Gleichstromstimulator mit Kathode und Anode (neuroConn DCSTIMULATOR).

Abbildung 3: Gleichstrom-Stimulator (neuroConn DC-STIMULATOR) mit am Dummy positionierter Kathode und Anode.

Abbildung 4: Schematische Darstellung der anodalen und kathodalen Stimulation..........................21

Abbildung 5: Mechanismus der Langzeitpotenzierung durch anodale tDCS ..................................23

Abbildung 6: Studienaufbau mit Anzahl an Patienten, die im Studienverlauf ausgeschlossen wurden und Zeitpunkt des Ausschlusses im Verlauf der Studie ...................................31

Abbildung 7: Durchschnittliche Anzahl der Migräneattacken im Intervall acht Wochen vor und acht Wochen nach tDCS für die kathodale und Placebogruppe

Abbildung 8: Anzahl der Migräne-assoziierten Tage 8 Wochen vor und 8 Wochen nach tDCS für die kathodale und Placebogruppe

Abbildung 9: Durchschnittliche Dauer der einzelnen Migräneattacken in Stunden acht Wochen vor und acht Wochen nach tDCS in der kathodalen und Placebogruppe.

Abbildung 10: Durchschnittliche Schmerzintensität auf einer Schmerzskala von null (kein Schmerz) bis drei (maximale Schmerzstärke) acht Wochen vor und acht Wochen nach tDCS für die kathodale und Placebogruppe 


\section{Tabellenverzeichnis}

Tabelle 1: Ausgangssituation nach Anamneseerhebung in beiden Stimulationsgruppen .33

Tabelle 2: Durchschnittliche Anzahl der Migräneattacken und Anzahl der Tage mit Migräne sowie Dauer in Stunden und Schmerzintensität im Vergleich der Patienten mit und ohne visuelle Aura

Tabelle 3: Prozentsatz an Patienten und Art der wahrgenommenen Nebenwirkungen während der tDCS .

Tabelle 4: Prozentsatz an Patienten und Art der wahrgenommenen Nebenwirkungen nach der tDCS. 


\section{Abkürzungsverzeichnis}

\begin{tabular}{|c|c|}
\hline AMPA & Aminomethylphosphonsäure \\
\hline ANOVA & analysis of variance (Varianzanalyse) \\
\hline BOLD-fMRI & blood oxygenation level-dependent functional magnetic resonance imaging \\
\hline cAMP & cyclisches Adenosinmonophosphat \\
\hline $\mathrm{CNV}$ & kontingente negative Variation \\
\hline CGRP & calcitonin gene-related peptide \\
\hline CSD & cortical spreading depression \\
\hline DLPFC & dorsolateraler präfrontaler Kortex \\
\hline FHM & familiäre hemiplegische Migräne \\
\hline GABA & Gamma-Aminobuttersäure \\
\hline EEG & Elektroenzelaphographie \\
\hline fMRI & funktionelle Magnetresonanztomographie \\
\hline IHS & International Headache Society \\
\hline LTD & Langzeitdepression \\
\hline LTP & Langzeitpotenzierung \\
\hline M1 & primär-motorische Rinde \\
\hline MA & Migräne mit Aura \\
\hline MEG & Magnetenzephalographie \\
\hline MEP & motorisch evozierte Potentiale \\
\hline MI & mean intensity \\
\hline MMP-9 & Matrix-Metalloproteinasen \\
\hline $\mathrm{MO}$ & Migräne ohne Aura \\
\hline MRT & Magnetresonanztomographie \\
\hline MT & motor threshold (Motorschwelle) \\
\hline $\mathrm{NaCl}$ & Natriumchlorid \\
\hline NAS & numerische Analogskala \\
\hline NMDA & N-Methyl-D-Aspartat \\
\hline PET & Positronen-Emissions-Tomographie \\
\hline PT & phosphene threshold \\
\hline rTMS & repetitive transkranielle Magnetstimulation \\
\hline tDCS & transkranielle Gleichstromstimulation (transcranial direct current stimulation) \\
\hline TMS & transkranielle Magnetstimulation \\
\hline VEP & visuell evozierte Potentiale \\
\hline ZNS & zentrales Nervensystem \\
\hline
\end{tabular}




\section{$1 \quad$ Einleitung und Zielsetzung}

\section{$1.1 \quad$ Überblick}

Die Migräne gehört zu den häufigsten Krankheitsbildern in der Neurologie. Das typische Symptom ist der rezidivierend auftretende intensive Kopfschmerz mit überwiegend einseitiger Lokalisation, welcher häufig zusammen mit vegetativen Begleitsymptomen auftritt. Die Kopfschmerzen und Begleiterscheinungen haben nicht selten eine einschränkende Wirkung auf den beruflichen und privaten Alltag des Patienten und können so zu einem enormen Leidensdruck führen. Zudem verursacht Migräne insbesondere durch die krankheitsbedingten Arbeitsausfälle beachtliche sozioökonomische Kosten.

Die Medikamente, welche zur Akuttherapie oder Prophylaxe eingesetzt werden, können die Symptome abschwächen oder unterbinden, jedoch die Krankheit nicht heilen. Für Patienten, die häufig oder unter sehr schweren Migräneattacken leiden, wird eine prophylaktische Behandlung empfohlen. Die bisher eingesetzten Medikamente haben sich in der Prophylaxe als effektiv erwiesenen. Dennoch ist ihre Wirksamkeit begrenzt und die Medikamente nicht immer verträglich, so dass komplementäre, nicht medikamentöse Therapieformen von großem Interesse sind. Vor diesem Hintergrund ist es verständlich, dass Alternativverfahren zur Migräneprophylaxe Thema intensiver wissenschaftlicher Forschung sind.

Die Patienten, die an Migräne leiden, zeigen eine veränderte Erregbarkeit des visuellen Kortex (Stankewitz und May 2007). Diverse Studien weisen daraufhin, dass Migräne durch eine vorbestehende gesteigerte Erregbarkeit des zentralen Nervensystems ausgelöst wird (Brigo et al. 2013). tDCS (transcranial direct current stimulation) ist eine nicht invasive, nebenwirkungsarme Methode für die Stimulation des Gehirns, welche eine nachhaltige Modulation der neuronalen Erregbarkeit und des Aktivitätszustandes im Gehirn über Veränderungen des neuronalen Membranpotentials bewirken kann (Nitsche und Paulus 2007). Therapeutisch kann diese Methode insbesondere bei Erkrankungen eingesetzt werden, bei denen eine veränderte kortikale Erregbarkeit als ursächlich angenommen wird. Ergebnisse aus Studien zur erfolgreichen Anwendung von tDCS bei Schmerzpatienten sprechen für die therapeutische Wirksamkeit (Fregni et al. 2006b; Fregni et al. 2006a; Antal et al. 2010; Marlow et al. 2013; Bolognini et al. 2015). Gegenwärtig ist nur unzureichend untersucht, ob tDCS bei Migräne eine wirksame prophylaktische Behandlung darstellen könnte.

Im Rahmen dieser Studie sollte bei Patienten mit Migräne mit und ohne Aura untersucht werden, ob eine standardisierte Behandlung mit tDCS über dem primär visuellen Kortexareal V1 eine wirksame Prophylaxe im Vergleich mit Placebo darstellt. Es wurde untersucht, inwieweit die Anzahl der Krankheitstage, die Dauer der Attacke sowie die Schmerzintensität unter der Behandlung versus Placebo reduziert werden können. 
Über einen Zeitraum von sechs Wochen wurde dreimal pro Woche über V1 eine Scheinoder kathodale Stimulation durchgeführt. Während der ersten drei Wochen erhielten alle Patienten eine Placebostimulation, in den nachfolgenden drei Wochen bekam nur die Hälfte der Patienten eine kathodale Stimulation. Im Rahmen eines Migränetagebuches wurden zwei Monate vor Beginn und nach Beendigung sowie während der Stimulation sowohl die Häufigkeit und Dauer der Migräneattacken als auch die Schmerzintensität und die Anzahl der Krankheitstage gemessen.

\subsection{Grundlagen}

\subsubsection{Historischer Überblick}

Die Krankheit Migräne ist kein Phänomen der Neuzeit. Schon im Zeitalter der Antike schilderte Hippokrates typische Symptome einer Migräne mit visueller Aura (Ritter 1969; Hagner 1994).

Eine umfassende Darstellung des Krankheitsbildes Migräne, welches er als „Cephalaea“ bezeichnete, beschrieb Aretaeus von Kappadokien im ersten Jahrhundert n. Chr. (Hagner 1994; Göbel 2012). Der römische Arzt Claudius Galenus (131-201 n. Chr.) führte den Begriff „Hemikrania“ für den halbseitigen Kopfschmerz ein, von dem sich der heutige Begriff Migräne ableitet (Herzog 2008; Hagner 1994; Hucklenbroich und Evers 2002; Göbel 2012). Er entwickelte auch erste pathophysiologische Vorstellungen, indem er Migräne gemäß der „Viersäftelehre“ auf ein Ungleichgewicht vorhandener Körperflüssigkeiten zurückführte (Herzog 2008; Hagner 1994; Göbel 2012).

In der zweiten Hälfte des 17. Jahrhunderts entstand eine Migränetheorie, die bis heute Aktualität besitzt. Thomas Willis (1621-1675) glaubte, dass die Pathologie des Migränekopfschmerzes durch einen Blutstau in verengten zerebralen Gefäßen begründet liegt, wodurch es zu einer lokalen Schwellung der Gefäßwand kommt. Diese Schwellung verursacht wiederum eine Dehnung von Nervenfasern und löst dadurch den Kopfschmerz aus (Hagner 1994; Weichselbaum 2004).

Die Theorie, dass die Ursache für Migräne vaskulär bedingt ist, wurde auch von Johann Jakob Wepfer (1620-1695) postuliert. Er führte die Ursache der Krankheit auf eine Vasodilatation mit gesteigerter Pulsation zurück (Schiller 1975; Hucklenbroich und Evers 2002). Wepfer war zudem der Ansicht, dass die Weiterleitung des Kopfschmerzes durch den Austritt von Serum aus Gefäßen über den ersten Trigeminusast erfolge (Ensink 1994; Weichselbaum 2004). Der Theorie der gefäßbedingten Entstehung der Migräne wurde im 19. Jahrhundert durch Emil du Bois-Reymond (1818-1896) und später auch durch Edward Liveing (1843-1907) eine neurogene Migränetheorie hinzugefügt. Emil du Bois-Reymond erachtete eine übermäßige Aktivität des sympathischen Nervensystems mit darauffolgender Verengung der Gefäße als Ursache der Krankheit (Göbel 2004). Liveing sah die Pathogenese 
der Krankheit in einer Instabilität des zentralen Nervensystems begründet (Hucklenbroich und Evers 2002; Keidel 2007).

Neben den zuvor dargestellten Theorien zur Pathogenese der Migräne sind im 20. Jahrhundert noch weitere Erklärungsmodelle entwickelt worden, die eventuell einen Teil der komplexen Struktur darstellen, aber den pathophysiologischen Mechanismus nicht erklären können. Wahrscheinlich handelt es sich um eine Kombination verschiedener Ursachen, welche für die Entstehung und die Klinik dieser komplexen Schmerzerkrankung verantwortlich sind.

\subsubsection{Epidemiologie}

Die Migräne ist heute mit einer weltweiten Prävalenz von ca. 12-15\% für Frauen und ca. 57\% bei Männern eine der häufigsten neurologischen Erkrankungen (Limmroth und Diener 2003b). Diese Zahlen variieren jedoch je nach verwendeter Untersuchungsmethode und Studienkollektiv in verschieden Studien zur Prävalenz der Migräne. Häufig tritt Migräne bei beiden Geschlechtern in der Pubertät das erste Mal auf, kann aber auch bereits bei Kindern vorkommen. Bei Kindern ist die Prävalenz in beiden Geschlechtern ausgewogen und steigt ab der Pubertät bei Frauen stärker als bei Männern an. Ab dem 45. Lebensjahr nimmt die Prävalenz bei beiden Geschlechtern wieder ab (Herzog 2008; Stewart et al. 1992). Zudem gibt es Hinweise, dass geographische Prävalenzen vorliegen, wobei die Häufigkeit bei Afrikanern und Asiaten niedriger als bei Europäern zu sein scheint (Herzog 2008; Breslau und Rasmussen 2001; Weichselbaum 2004).

Studien zum Einfluss des sozialen Status auf die Häufigkeit der Migräne kommen zu unterschiedlichen Ergebnissen. Während einige Studien keinen Unterschied darlegen (Rasmussen 2001), zeigen andere Studien, dass die Prävalenz der Migräne mit dem sozioökonomischen Status auf inverse Weise assoziiert zu sein scheint (Stewart et al. 1992). Diese Ergebnisse könnten dafür sprechen, dass die Pathogenese der Migräne multifaktoriell ist und neben der genetischen Komponente eventuell auch soziokulturelle und umweltbedingte Faktoren eine Rolle spielen (Stewart et al. 1996; Weichselbaum 2004).

\subsubsection{Klassifikation, Klinik und Diagnostik der Migräne}

Die Einteilung der verschiedenen Migräneformen erfolgt nach der Klassifikation der International Headache Society (IHS) (Göbel 2012). In dieser Klassifikation wird anhand bestimmter Diagnosekriterien wie dem Vorhandensein von Begleitsymptomen, Dauer, Genetik oder Lokalisation in weitere Untergruppen unterschieden. Die beiden häufigsten Formen stellen dabei die Migräne ohne Aura (MO) und die Migräne mit Aura (MA) dar.

Das klinische Charakteristikum der MO ist ein attackenartig auftretender, starker, zumeist unilateraler (zwei Drittel aller Patienten), aber auch beidseitig (überwiegend im Kindesalter) auftretender, pulsierender Kopfschmerz. Typischerweise gehen die Kopfschmerzen mit 
vegetativen Begleitsymptomen wie Licht-, Lärm- und Geruchsempfindlichkeit sowie Übelkeit und Erbrechen einher (Limmroth und Diener 2003b). Der Schmerz lokalisiert sich meist in der Temporalregion, im okzipitonuchalen Übergangsbereich oder der periorbitalen-frontalen Region (Diener 2002). Die wiederkehrenden Kopfschmerzattacken dauern zwischen 472 Stunden an und werden durch körperliche Aktivität verstärkt (Gleixner et al. 2007/08).

Bei ca. 10-15\% aller Patienten ist der Migräneanfall mit einer Aura kombiniert (Limmroth und Diener 2003a). Unter dem Begriff Aura werden unterschiedliche fokale neurologische Symptome zusammengefasst. Bei Patienten mit MA tritt am häufigsten eine visuelle Aura (Sehstörungen, Skotome) auf, es kann aber auch zu anderen Reiz- und Ausfallserscheinungen, wie Sensibilitätsstörungen, motorischen Ausfällen und neuropsychologischen Defiziten, kommen (Limmroth und Diener 2003b; Limmroth und Diener 2003a). Selten kommt es, wie bei der autosomal-dominant vererbten familiär hemiplegischen Migräne, zur Ausbildung von motorischen Paresen oder Sprachstörungen (Göbel 2012) (siehe 1.2.4.1).

Die Symptome einer Aura entwickeln sich innerhalb eines Zeitraumes von 5-20 Minuten und halten meist nicht länger als 60 Minuten an. Der typische Kopfschmerz folgt der Aura meist innerhalb der nächsten 60 Minuten, kann aber auch vor oder parallel mit der Aura einsetzen. Selten kann eine Aura auch isoliert ohne Kopfschmerzen auftreten (Limmroth und Diener 2003a). Von der Aura abzugrenzen sind dagegen unspezifische Vorbotensymptome wie Übelkeit, Heißhunger, Stimmungsschwankungen, Polyurie, Müdigkeit und andere sehr individuelle Symptome, die sowohl bei MO als auch MA Tage bis Stunden vor dem eigentlichen Anfall auftreten können (Limmroth und Diener 2003a).

Faktoren, die für das Auslösen eines Anfalls eine Rolle spielen, sind individuell sehr unterschiedlich. Als mögliche Triggerfaktoren kommen hormonelle Schwankungen im Rahmen des Menstruationszyklus, Stress, aber auch die Entspannungsphase nach Anstrengung, verändertem Schlaf-Wach-Rhythmus, Wetterwechsel, Muskelverspannungen im Nackenbereich, Alkoholgenuss sowie der Verzehr von bestimmten Nahrungsmitteln (z. B. Käse, Schokolade, Zitrusfrüchte) und weitere Faktoren in Betracht (Limmroth und Diener 2003a; Kelman 2007).

Die Diagnose der Migräne wird allein klinisch anhand der typischen Anamnese und des spezifischen Schmerzcharakters gestellt (Evers et al. 2008). Nach Kriterien der IHS müssen fünf Migräneanfälle bei MO bzw. zwei Migräneanfälle bei MA vorliegen. Die Anamnese, die allgemeine körperliche und neurologische Untersuchung müssen dabei eine sekundäre Ursache der Kopfschmerzen ausschließen (Olesen 2006).

Zwischen Migräne und anderen Erkrankungen gibt es Assoziationen. Als Komorbiditäten zur Migräne gelten der Schlaganfall, Depressionen, Angsterkrankungen, Epilepsien und Allergien (Diener und Leniger 2003). 


\subsubsection{Genetik}

Eine familiäre Häufung von Migräne ist schon lange bekannt, so dass die Vermutung einer genetischen Disposition naheliegt. In Zwillingsstudien konnten signifikant höhere Inzidenzen für Migräne bei eineiigen im Vergleich zu zweieiigen Zwillingen festgestellt werden (Gervil et al. 1999; Ulrich et al. 1999; Ferrari et al. 2006). Es wird von einer genetischen Komponente ausgegangen, die bei den MA-Patienten höher zu liegen scheint als bei den MO-Patienten (Pietrobon und Striessnig 2003).

In den zahlreichen Studien zur Genetik der Migräne konnten zunächst nur bei der familiär hemiplegischen Migräne (FHM) eindeutige Gendefekte als Ursache für die Migräne identifiziert werden. In neueren Arbeiten konnten auch - für Migräne mit und ohne Aura - Gendefekte lokalisiert werden, die mit einer erhöhten Erkrankungswahrscheinlichkeit assoziiert sind. So zeigten Anttila et al., dass die Genvariante rs1835740 auf Chromosom 8 zu einem deutlich erhöhtem Risiko führt, eine Migräne mit und ohne Aura zu entwickeln (Anttila et al. 2010). Die Lokalisation der veränderten Gensequenz zwischen den beiden Genen PGCP und MTDH/ AEG-1, welche die Menge des Nervenüberträgerstoffes Glutamat regulieren, legt nahe, dass durch die Genveränderung die Glutamatkonzentration verändert ist. In einer weiteren groß angelegten genomweiten Studie von Antilla wurden Einzelnukleotid-Polymorphismen verglichen. Als Ergebnis dieser Arbeit konnten sieben bekannte Risikogene bestätigt und fünf bisher nicht bekannte Risikogene sowie 134 mit der Erkrankung möglicherweise assoziierte Genloci entdeckt werden (Anttila et al. 2013).

Fasst man zusammen, so kann davon ausgegangen werden, dass es sich bei Migräne um ein multifaktorielles Krankheitsbild mit einer polygenen Vererbung handelt, wobei die phänotypische Ausprägung individuell sehr unterschiedlich ist.

\subsubsection{Die familiär hemiplegische Migräne (FHM) als Modellerkrankung für die häufigen Migräneformen}

Die Ergebnisse vieler Forschungsarbeiten deuten auf eine genetisch bedingte Prädisposition hin. Anhand der FHM können diesbezüglich möglicherweise Rückschlüsse auf die Pathophysiologie auch der häufigeren Migräneformen gewonnen werden (Moskowitz et al. 2004).

Die FHM ist ein seltener, autosomal-dominant vererbter Subtyp der Migräne mit Aura, der wiederum in drei Unterklassen unterteilt wird. Charakteristisch für die Aura bei allen drei Subtypen der FHM ist zusätzlich zu den visuellen und sensorischen Phänomenen das Auftreten von reversiblen motorischen Störungen wie Paresen oder Sprachstörungen (Göbel 2012).

Für die FHM konnten Mutationen auf Genabschnitten gefunden werden, die Ionenkanäle im zentralen Nervensystem kodieren. Die mutationsbedingt veränderte Struktur der Ionenkanäle kann auf verschiedene Art und Weise zu Instabilitäten in der Verschaltung des zentralen Nervensystems führen. Die Mutationen sowohl bei der FHM1 als auch bei der FHM2 
führen zu einer Erhöhung des exzitatorischen Transmitters Glutamat im synaptischen Spalt (Hans et al. 1999; De Fusco et al. 2003), wodurch es zu einer erniedrigten Schwelle für eine Cortical Spreading Depression (CSD) (siehe 1.2.5.5) kommt.

Die Studien zu den bisher gefundenen Mutationen bei der FHM3 zeigten Änderungen in der Kanalkinetik, die eine elektrophysiologische Übererregbarkeit bedingen (Dichgans et al. 2005; Vanmolkot et al. 2007; Kahlig et al. 2008), welche wiederum die Entstehung und die Fortleitung einer CSD begünstigen könnten. Durch die gewonnenen Erkenntnisse zur Pathophysiologie der FHM lässt sich vielleicht auch die Pathophysiologie der häufigen Migräneformen besser verstehen. Möglicherweise können auch andere Formen der Migräne auf eine genetische Disposition im Sinne eines Ionenkanaldefektes mit nachfolgend gesteigerter elektrophysiologischer Erregbarkeit zurückgeführt werden (Limmroth und Diener 2003b; Wiescher 2008).

\subsubsection{Pathophysiologie}

Obwohl die Migräne schon lange erforscht wird, ist ihre genaue Ursache noch immer nicht wirklich geklärt. Es wird davon ausgegangen, dass verschiedene Mechanismen auf unterschiedlichen anatomischen Ebenen ineinander greifen.

\subsubsection{Schmerzentstehung bei Migräne}

Die eigentliche Schmerzentstehung im Gehirn können nur wenige Strukturen generieren. Zu diesen gehören die basalen Arterien, die venösen Sinus, die meningealen Blutgefäße sowie die Dura mater. Alle genannten Strukturen werden durch den Nervus trigeminus innerviert. Die Nervenfasern werden im Falle eines Migräneanfalles durch die Freisetzung von Neuropeptiden aktiviert und führen dann durch Weiterleitung des Signals in die Großhirnrinde zum Schmerzempfinden (Ebersberger 2002; Limmroth und Diener 2003a). Welche Hirnstrukturen genau dabei die Aktivierung der trigeminalen Fasern verursachen, ist bislang jedoch noch nicht ausreichend geklärt.

\subsubsection{Veränderungen des Blutflusses}

Erste Theorien zur Pathophysiologie der Migräne gingen von einem primär vaskulär bedingten Kopfschmerz aus. Dies beruhte auf der Annahme, dass eine initiale Vasokonstriktion der kranialen Gefäße eine reaktive schmerzhafte Vasodilatation zur Folge haben sollte. Es ist aber nicht abschließend untersucht, ob die vaskulären Veränderungen als Ursache für die neuronale Aktivität anzusehen oder lediglich als deren Begleiterscheinungen zu werten sind (Olesen et al. 1990). Limmroth et al. konnten zudem darlegen, dass nach Gabe von vasokonstriktiven Substanzen wie Sumatriptan der Blutfluss anstieg, dies jedoch nicht zu einer Abnahme des Kopfschmerzes führte (Limmroth et al. 1996). Weitere Studien zeigten, dass sich der Blutfluss während einer Migräneattacke ohne Aura im Vergleich zum migränefreien Intervall nicht signifikant veränderte (Friberg et al. 1991; Caekebeke et al. 1992; Limmroth 
et al. 1996; Limmroth und Diener 2003b). Daher lässt sich die Entstehung des Migränekopfschmerzes nicht alleinig durch die Veränderung des Durchmessers der Hirngefäße und damit verbundenen Variation des Blutflusses erklären (Limmroth und Diener 2003b).

\subsubsection{Neurogene Entzündung}

Tierexperimentelle Studien gaben Hinweise auf ein neurogen bedingtes Entzündungsgeschehen, welches in mehreren Komponenten einer aseptischen Entzündung entspricht. Es kommt zu einer Vasodilatation meningealer Gefäße, Permeabilitätssteigerung mit Plasmaextravasation in das umgebende Gewebe und zur Degranulation von Mastzellen mit einer Freisetzung von Entzündungsmediatoren wie Serotonin, Histamin, Prostaglandinen etc. Studien zur Peptidfreisetzung zeigten bei Migränepatienten, dass während einer Migräneattacke die Konzentration von vasoaktiven Neuropeptiden, insbesondere des Calcitonin Gene-Related Peptide (CGRP), deutlich ansteigt. Durch die Gabe von Dihydroergotamin oder Sumatryptanen, beides in der Akuttherapie der Migräne erfolgreich verwendete Medikamente, konnte der Plasmaspiegel wiederum gesenkt werden (Goadsby und Edvinsson 1993; Edvinsson und Goadsby 1994; Limmroth und Diener 2003b). Aufgrund dieser Erkenntnisse wird davon ausgegangen, dass es während eines Migräneanfalls zu einer Aktivierung trigeminaler Fasern mit Freisetzung von vasoaktiven Neuropeptiden kommt.

\subsubsection{Aktivierung im Hirnstamm}

Eine wichtige Bedeutung für die Entstehung einer Migräneattacke vermutet man heute auch im Hirnstamm. In Tierexperimenten konnten einzelne Hirnstammkerne identifiziert werden, bei denen es nach Stimulation zu einer Änderung des zerebralen Blutflusses kam (Kaube et al. 1992; Kaube et al. 1993; Limmroth und Diener 2003b). Bei Migränepatienten konnten übereinstimmend dazu mittels Positronen-Emissions-Tomographie (PET) eine deutliche Aktivierung dieser Hirnstammbereiche um das periaquäduktale Grau während der Migräneattacke nachgewiesen werden. Dieses Phänomen konnte aber nicht bei Schmerzen, welche durch andere Ursachen als Migräne ausgelöst wurden, beobachtet werden und gilt daher als spezifisch für Migräne (Weiller et al. 1995; Bahra et al. 2001; Limmroth und Diener 2003b). Der Bereich um das periaquäduktale Grau war nach Gabe von Sumatriptan weiterhin aktiviert. Erst im schmerzfreien Intervall bildete sich die Aktivität im Bezirk um das periaquäduktale Grau auf das Ausgangsniveau zurück. Daher kann angenommen werden, dass diese Aktivierung kein Begleiterscheinung der Schmerzwahrnehmung ist, sondern im Kontext mit der Entwicklung der Migräneattacke steht (Limmroth und Diener 2003b). Funktionell gehört die Region des periaquäduktalen Graus zum antinozizeptiven System, indem es inhibitorisch auf die Erregungsweiterleitung wirkt (Knight und Goadsby 2001; Limmroth und Diener 2003b). Durch eine Dysfunktion dieses Systems kann es zu einer Reduktion der Schmerzschwelle kommen. Demnach wurden Fallberichte bekannt, in denen Hirnstammläsionen in Höhe des periaquäduktalen Graus zu neu aufgetretenen Migräneanfällen auf der kontralateralen Hemisphäre führten (Raskin et al. 1987; Haas et al. 1993; Limmroth und Diener 2003b). 


\subsubsection{Cortical Spreading Depression}

Die CSD ist charakterisiert durch eine sich durch den Kortex fortbewegende Depolarisationswelle, die als elektrophysiologisches Korrelat wandernder Flimmerskotome angesehen wird (Lauritzen 1994; Richter und Lehmenkühler 2008; Eikermann-Haerter und Ayata 2010). Zu Beginn der CSD steigt das extrazelluläre Kalium von 3 auf bis zu $60 \mathrm{mmol} / \mathrm{l}$ an (Grafstein 1963; Richter und Lehmenkühler 2008; Kotzian 2009). Gleichermaßen kann auch experimentell durch lokale Applikation von Kalium eine CSD hervorgerufen werden (Bureš et al. 1974; Richter und Lehmenkühler 2008; Kotzian 2009). Der Anstieg der Kaliumkonzentration bewirkt dann die Depolarisation von umliegenden präsynaptischen Nervenendigungen. Durch die Depolarisation öffnen sich spannungsabhängige Kationenkanäle, wodurch sich die Erregung weiter ausbreiten kann (spreading). Der folgende Natrium- und Kalziumeinstrom in die Zelle führt zur Freisetzung von Neurotransmittern wie Glutamat (Kow und van Harreveld 1972; Kraig und Nicholson 1978).

Der Depolarisation folgt eine neuronale Untererregbarkeit (Hyperpolarisation). Einhergehend mit der CSD ändert sich parallel zum Erregungszustand der kortikale Blutfluss in der entsprechenden Region. Während der neuronalen Depolarisation kommt es zu einer temporären Vasodilatation in pialen und kortikalen Blutgefäßen, die einen Anstieg des regionalen Blutflusses bewirkt (cortical spreading hyperaemia). Auf die im Anschluss daran folgende neuronale Untererregbarkeit schließt sich eine Reduktion des kortikalen Ruheblutflusses (cortical spreading oligaemia) an, bis anschließend wieder der Ausgangswert erreicht wird (Duckrow 1991; Lauritzen 1994).

Durch das bildgebende Verfahren der BOLD-fMRI (blood oxygenation level-dependent functional magnetic resonance imaging) konnte ein direkter Zusammenhang zwischen Migräneaura und CSD dargelegt werden (Cao et al. 1999; Hadjikhani et al. 2001). Zeitgleich mit einer visuellen Aura wurde eine sich über den okzipitalen Kortex ausbreitende Signalveränderungen dargestellt, welche sich mit einer Geschwindigkeit von 3,5 \pm 1,1 $\mathrm{mm} / \mathrm{min}$ parallel zu der Ausbreitung der Migräneaura im Gesichtsfeld des Patienten über den Kortex bewegte (Hadjikhani et al. 2001; Richter und Lehmenkühler 2008; Kotzian 2009). Währenddessen konnte eine initiale, kurzzeitige Hyperperfusion mit nachfolgender Hypoperfusion registriert werden. Die Hypoperfusion mit einem Ausmaß von bis zu 50\% wirkt jedoch nicht wie eine Ischämie, sondern im Sinne eines neurovaskulären Mechanismus (Cutrer et al. 1998; Limmroth und Diener 2003b; Wiescher 2008).

Der CSD entsprechende Wellen neuronaler Erregung konnten auch mit Magnetenzephalographie (MEG) während visuell induzierter Auren registriert werden (Bowyer et al. 2001; Richter und Lehmenkühler 2008). Diese im MRT und MEG beobachteten Erregungen wurden jedoch nicht bei gesunden Kontrollprobanden festgestellt. Dies kann als Ausdruck gesteigerter neuronaler Erregbarkeit bei Migränepatienten interpretiert werden, wobei die Gründe für diese veränderte neuronale Erregbarkeit noch nicht ausreichend geklärt sind (Richter und Lehmenkühler 2008). 
Eine Ursache liegt wahrscheinlich in einem gestörten Transportsystem für die Speicherung bzw. die Wiederaufnahme von Glutamat. Der erhöhte Plasmaspiegel von Glutamat, welcher auch teilweise bei den durch Gendefekte hervorgerufenen Migräneformen vorliegt, führt zu einer Aktivierung der NMDA-Rezeptoren. Die Aktivierung der NMDA-Rezeptoren führt zu einer Sensibilisierung der Nervenzellen, wodurch es nachfolgend zu der erhöhten neuralen Erregbarkeit kommen kann (Göbel 2012). Die orale Einnahme oder die Applikation von Glutamat sowie andere Vorgänge, die das neuronal-gliale Gleichgewicht lokal stören und zur Freisetzung von Glutamat führen, können eine CSD auslösen (van Harreveld 1959; Bureš et al. 1974; Richter und Lehmenkühler 2008; Göbel 2012). Zudem weisen Migränepatienten, insbesondere Patienten mit MA, während der Attacke signifikant erhöhte Glutamat- und Aspartat-Plasmaspiegel auf, wodurch es zu einer Aktivierung des NMDA-Rezeptors kommt (Göbel 2012). Auch der während der Migräneattacke reduzierte Magnesiumspiegel führt zu einer erhöhten Empfindlichkeit des NMDA-Rezeptors (Göbel 2012).

Ein weiterer Grund für die Entstehung und Ausbreitung der CSD könnte auch eine Dysfunktion von Gliazellen sein. Gliazellen besitzen eine Pufferfunktion, die für die Wiedereinstellung des Kaliumgleichgewichts im Extrazellulärraum sehr wichtig ist (Paulson und Newman 1987). Möglicherweise spielt eine veränderte Enzymfunktion bei Astrozyten, einem Subtyp der Gliazellen, eine Rolle in der Pathogenese. Brennan et al. konnten bei zwei Familien mit ungewöhnlicher Häufung der Migräne eine Mutation im Gen für die Caseinkinase I delta finden. Durch eine herabgesetzte Funktion des Enzyms Caseinkinase I delta könnte die Konzentration von Kalzium und nachfolgend die Signalwirkung auf benachbarte Nervenzellen verändert sein. Mäuse, die mit dem defekten Gen ausgestattet wurden, zeigten eine deutlich erniedrigte Schwelle für die Ausbildung einer CSD und eine verstärkte arterielle Dilatation während der CSD (Brennan et al. 2013).

Ob die CSD auch den Kopfschmerz bei der Migräne generieren kann, ist nicht genau erforscht. Es konnte belegt werden, dass die CSD in der Lage ist, trigeminale Fasern zu aktivieren und die Freisetzung von vasoaktiven Neuropeptiden zu verursachen (Bolay et al. 2002; Wiescher 2008; Costa et al. 2013). Gursoy-Ozdemir et al. beobachteten in Tierexperimenten eine durch CSD induzierte Erhöhung der Matrix-Metalloproteinasen (MMP-9). Dies führt $\mathrm{zu}$ einer Störung der Blut-Hirn-Schranke und einer Extravasation von Plasmaproteinen (Gursoy-Ozdemir et al. 2004). Auch dieser Mechanismus könnte zu einer Aktivierung nozizeptiver trigeminaler Fasern der Meningen beitragen. Aus diesen Erkenntnissen kann abgeleitet werden, dass die CSD das schmerzsensitive trigeminale Nervensystem aktivieren kann und somit möglicherweise auch zur Generierung des Kopfschmerzes beitragen könnte.

Studien über die Wirkung von Medikamenten zur Migräneprophylaxe unterstützen die Vermutung, dass die CSD mitverantwortlich für die Generierung des Migränekopfschmerzes sein kann. Die mehrwöchige Vorbehandlung von Propanolol, Topiramat, Methysergid, Valproinsäure und Amitriptylin im Tierexperiment führte zu einer Reduktion der kaliuminduzierten CSD (Ayata et al. 2006). Wie in der genannten Studie, zeigen Medikamente zur 
Migräneprophylaxe im klinischen Alltag meist erst nach mehreren Monaten eine ausreichende Wirksamkeit. Wahrscheinlich verhindern die zur Prophylaxe eingesetzten Medikamente durch eine veränderte Genexpression oder durch direktes Einwirken auf die neuronale Zellmembran, eine elektrische Ausbreitung der CSD. Damit entsprechend hohe Plasmakonzentrationen erreicht und pharmakodynamische Prozesse erfolgen können, wird eine längere Zeitspanne benötigt (Kotzian 2009; Costa et al. 2013).

Nach tierexperimentellen Studien erhärten nun auch erste klinische Studien die Hypothese, dass NMDA-Antagonisten in der Prophylaxe der Migräne wirksam sind (Charles et al. 2007; Göbel 2012). Auch in der Verwendung dieser Medikamentengruppe könnte der Wirkmechanismus in der Blockade der CSD bestehen (Peeters et al. 2007; Göbel 2012).

\subsubsection{Gestörte Habituation des zentralen Nervensystems}

Patienten mit Migräne scheinen auch im migränefreien Intervall eine gesteigerte Empfindlichkeit gegenüber Umweltreizen aufzuweisen. Neurophysiologischen Untersuchungen zur Erregbarkeit des zentralen Nervensystems weisen darauf hin, dass bei Patienten mit Migräne möglicherweise eine Übererregbarkeit des zentralen Nervensystems besteht. So konnten in Studien Abnormitäten im EEG, wie zum Beispiel in der Ableitung der kontingenten negativen Variation (CNV), aufgezeigt werden (Göbel 2012). Bei der kontingenten negativen Variation handelt es sich um eine Negativierung, die sich im EEG darstellen lässt, wenn auf einen Reiz eine motorische Reaktion erfolgen soll. Die CNV kann dementsprechend als Ausdruck für kortikale Vorbereitungsprozesse verstanden werden (Werthmann und Wieting 2005) und eignet sich aufgrund dessen zur Analyse von kortikalen Aktivierungsprozessen während der Vorbereitung auf eine motorische Antwort (Göbel 2012; Jonas 2012). Bei Patienten mit Migräne ohne Aura konnte im kopfschmerzfreien Intervall eine erhöhte Amplitude und eine herabgesetzte Habituation der CNV dargestellt werden (Maertens de Noordhout et al. 1986; Kropp et al. 1996; Göbel 2012; Jonas 2012). Dies kann als Hinweis auf eine kortikale Übererregbarkeit bei Patienten mit Migräne interpretiert werden. Durch die Therapie mit Betablockern, ein in der Prophylaxe der Migräne wirksames Medikament, konnte die Amplitude gesenkt und die Habituation der CNV verbessert werden (Schoenen et al. 1986; Siniatchkin et al. 2007; Göbel 2012; Jonas 2012).

\subsubsection{Erregbarkeit des visuellen Systems}

Der visuelle Kortex ist im Bereich des Okzipitallappens lokalisiert. Nach Brodmann entsprechen ihm die Area 17, 18 und 19, welche wiederum in die primäre Sehrinde V1 (Area 17) und die sekundäre Sehrinde V2 bis V5 (Area 18 und 19) eingestuft werden (Schiebler 2005; Trepel 2004). 


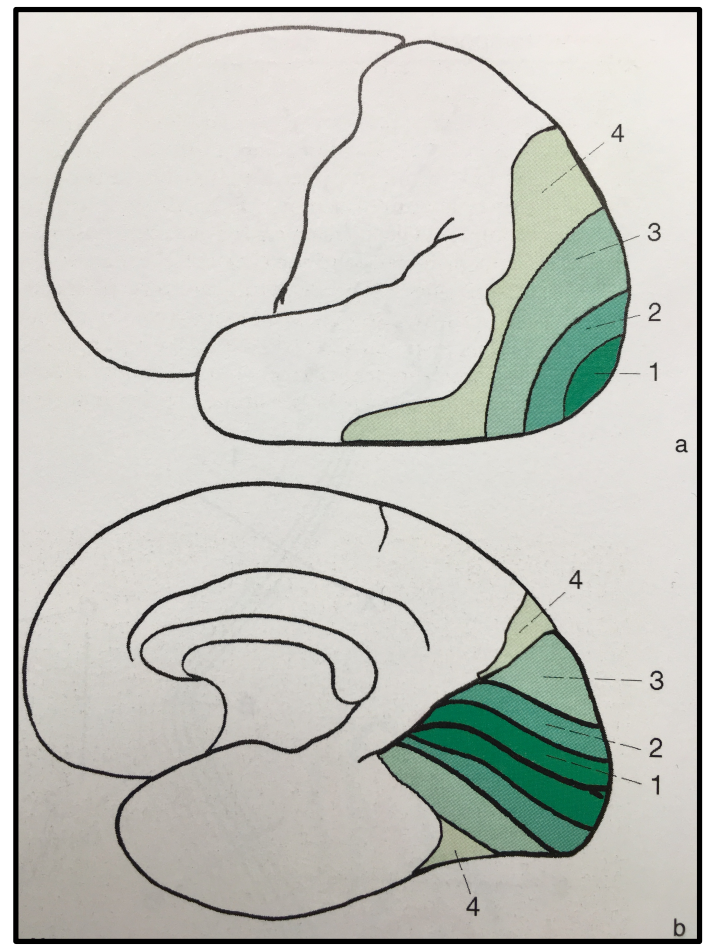

Abbildung 1: visuelle Rindenfelder. a) von lateral; b) von medial 1 Primäre Sehrinde (Area 17), 2 und 3 „klassische“ sekundäre Sehrinde, 2 Area 18, 3 Area 19, 4 Lokalisation weiterer, übergeordneter visueller Rindenfelder pel 2004; die Verwendung erfolgt mit freundlicher Genehmigung von Elsevier.)

Viele Studien zur Pathophysiologie der Migräne konnten zeigen, dass Patienten, die regelmäBig an Migräne leiden, eine veränderte Erregbarkeit des visuellen Kortex aufweisen. Unterschiedliche Forschungsarbeiten zur Erregbarkeit des visuellen Kortex bei Migräne erbrachten variable und teilweise heterogene Ergebnisse.

Sowohl Wilkins et al. als auch Huang et al. konnten in Studien zu Musterstimulationen mit Gittermustern bei Migränepatienten im Vergleich zu Kontrollprobanden intensivere Illusionen feststellen (Wilkins et al. 1984; Huang et al. 2003; Temme 2006).

In den vergangenen Jahren wurde die Erregbarkeit des visuellen Kortex vermehrt mittels transkranieller Magnetstimulation (TMS) untersucht. Messungen zur Auslösung von Phosphenen (Lichtblitzen), welche mittels TMS ausgelöst wurden, zeigten sehr heterogene Ergebnisse. So konnten sowohl signifikant niedrigere als auch signifikant höhere Schwellen bei Patienten mit Migräne im Vergleich zu gesunden Kontrollprobanden nachgewiesen werden (Afra et al. 1998a; Bohotin et al. 2003; Gerwig et al. 2005; Gunaydin et al. 2006; Temme 2006). Jedoch war in mehreren Studien, insbesondere bei Migränepatienten mit Aura, eine deutlich erniedrigte Schwelle für das Auslösen von Phosphenen darstellbar (Aurora et al. 1998; Aurora und Welch 2000; Mulleners et al. 2001b; Antal et al. 2005; Gerwig et al. 2005; Temme 2006; Brigo et al. 2012). Auch in einem Review von Brigo, in welchem 10 Studien mit insgesamt 277 Migränepatienten und 193 Kontrollprobanden verglichen wurden, zeigte sich wieder bei Migränepatienten mit Aura eine erniedrigte Phosphenschwelle (Brigo et al. 
2012). Die Daten bei Migränepatienten ohne Aura waren jedoch nicht bei der Applikation von jeder Stimulationsspule signifikant. Insgesamt sprechen die Ergebnisse für eine Übererregbarkeit des visuellen Kortex, insbesondere bei Migränepatienten mit Aura.

Zudem konnten erhöhte Amplituden von visuell evozierten Potentialen (VEP) (Gawel et al. 1983; Kahlil 1991) und eine herabgesetzte Unterdrückbarkeit der visuellen Wahrnehmung durch TMS gemessen werden (Palmer et al. 2000; Mulleners et al. 2001a; Temme 2006). Auch diese Ergebnisse können als Übererregbarkeit des okzipitalen Kortex bei Migräne gewertet werden.

Untersuchungen zur TMS-induzierten Wahrnehmungssuppression gaben Hinweise auf eine gestörte Modifizierbarkeit der Erregbarkeit des visuellen Kortex bei Patienten mit Aura (Siniatchkin und Bartsch 2007). So konnte ein TMS-Puls, welcher $100 \mathrm{~ms}$ nach einer Bildschirmpräsentation gegeben wurde, bei Patienten mit MA im Gegensatz zu MO und Gesunden kaum eine Unterdrückung der visuellen Wahrnehmung bewirken (Chronicle et al. 2006). Dies kann als mangelnde Inhibition des visuellen Kortex bei MA verstanden werden.

Studien zu Erregbarkeit des visuellen Kortex bei Migräne belegten bei Patienten eine gestörte Habituation (Schoenen et al. 1995; Afra et al. 1998b; Shepherd 2000). So zeigten Afra et al. und Schoenen et al., dass bei der wiederholten Ableitung visuell evozierter Potentiale (VEP) Migränepatienten im Gegensatz zu gesunden Kontrollen keine Habituation zeigten. Statt einer verminderten VEP-Amplitude konnte bei Migränepatienten auch nach mehren Minuten Stimulation ein weiterer Amplitudenanstieg beobachtet werden. Dieses Ergebnis kann zweideutig interpretiert werden. Von der Annahme ausgehend, dass Patienten mit Migräne von einem niedrigeren Aktivierungsniveau starten, wird die Schwelle, ab der eine Habituation beginnt, erst später erreicht als bei gesunden Kontrollprobanden. Dies würde auf eine Hypoexzitabilität hinweisen. Die ausbleibende Habituation kann aber auch als Hyperexzitabilität der Nervenzellen gedeutet werden (Temme 2006; Brighina et al. 2009).

Bei Untersuchungen zur Erregbarkeit des motorischen Kortex bei Migräne zeigen die meisten Studien eine normale Erregbarkeit des motorischen Kortex bei Migränepatienten (Siniatchkin und Bartsch 2007). Studien, die sowohl den motorischen als auch den visuellen Kortex untersuchten, zeigten für den motorischen Kortex eine normale, für den visuellen Kortex hingegen eine veränderte Erregbarkeit (Bohotin et al. 2003; Gunaydin et al. 2006).

In einigen Forschungsarbeiten konnte zudem gezeigt werden, dass sich die Reizverarbeitung und Reaktionsfähigkeit des zentralen Nervensystems bei Migränepatienten zwischen den Anfällen ändern kann (Judit et al. 2000; Antal et al. 2006; Siniatchkin et al. 2009). Hierbei wurden vor allem für den Zeitraum vor den Migräneattacken Auffälligkeiten in der Erregbarkeit festgestellt. Dies deutet auf eine dynamische Dysfunktion des visuellen Kortex hin. Auch der weibliche Hormonzyklus beeinflusst möglicherweise die visuelle Erregbarkeit (Smith et al. 1999; Smith et al. 2002; Boros et al. 2009). Aufgrund dessen scheint der Zeitpunkt der Datenerhebung im Kontext zum vorangegangenen bzw. darauffolgenden Migräneanfall bzw. zum weiblichen Hormonzyklus für die Auswertung von großer Bedeutung zu 
sein. Insgesamt scheint die kortikale Erregbarkeit bei Migräne mit Aura stärker verändert zu sein als bei Migräne ohne Aura (Siniatchkin und Bartsch 2007).

Zusammenfassend betrachtet sind die Untersuchungsergebnisse nicht eindeutig. Gründe dafür könnten sowohl in den methodischen Unterschieden der Studien als auch in der episodisch veränderbaren Erregbarkeit bei Migräne liegen (Brigo et al. 2012). Des Weiteren sind die Effekte einer akuten oder prophylaktischen Medikamenteneinnahme auf die kortikale Erregbarkeit und damit ihr Einfluss als Störfaktoren in Studien unklar. Zudem ist die Population der Migränepatienten sehr heterogen (Siniatchkin und Bartsch 2007). Als gesichert gilt die Annahme, dass Patienten mit Migräne ein Defizit in der Habituation und eine veränderte kortikale Erregbarkeit aufweisen, die sich während des Migränezyklus zu verändern scheint (Stankewitz und May 2009; Aderjan 2012).

\subsection{Therapie und Prophylaxe}

Die Ausführungen zum Thema Therapie und Prophylaxe stützen sich im wesentlichen auf die Leitlinie der Deutschen Gesellschaft für Neurologie von 2012 sowie die Zusammenfassung von Evers et al. (Evers et al. 2008; Deutsche Gesellschaft für Neurologie 2012). Die Indikation zur medikamentösen Therapie besteht bei mittelschwerer bis schwerer Migräne. Die Auswahl und Applikationsform der Medikamente richtet sich dabei nach Stärke der Anfälle, Begleitsymptomen, Begleiterkrankungen und Alter (Evers et al. 2008; Deutsche Gesellschaft für Neurologie 2012). Zur Auswahl für die medikamentöse Akuttherapie stehen Analgetika wie Acetylsalicylsäure, Ibuprofen, Diclofenac, Naproxen, Paracetamol und Metamizol, Derivate des Mutterkornalkaloids, Triptane sowie Antiemetika (MCP, Domperidon) zur Verfügung (Evers et al. 2008; Deutsche Gesellschaft für Neurologie 2012).

\subsubsection{Indikation und Ziel der Prophylaxe}

Die Indikation zu einer medikamentösen Prophylaxe der Migräne ergibt sich nach Leitlinien der Deutschen Gesellschaft für Neurologie von 2012 (Deutsche Gesellschaft für Neurologie 2012) bei besonderem Leidensdruck und Einschränkung der Lebensqualität. Zusätzliche Kriterien für den Beginn wird eine Migräneprophylaxe sind folgende:

- drei und mehr Migräneattacken pro Monat, die die Lebensqualität beeinträchtigen

- Migräneattacken, die regelmäßig länger als 72 Stunden anhalten

- Attacken, die auf eine Therapie entsprechend den gegebenen Empfehlungen zur Akuttherapie (inkl. Triptanen) nicht ansprechen

- Patienten, bei denen Kontraindikationen für die Einnahme von Triptanen bestehen und/oder

- wenn Nebenwirkungen der Akuttherapie nicht toleriert werden 
- bei Zunahme der Attackenfrequenz und Einnahme von Schmerz- oder Migränemitteln an mehr als zehn Tagen im Monat

- bei komplizierten Migräneattacken mit beeinträchtigenden (z. B. hemiplegischen) und/oder lang anhaltenden Auren

- Zustand nach migränösem Infarkt bei Ausschluss anderer Ursachen

Ziel der Prophylaxe ist die Senkung des Leidensdruckes und der Beeinträchtigung der Lebensqualität durch Reduzierung von Häufigkeit, Schwere und Dauer der Migräneattacken. Zudem bewirkt eine Prophylaxe eine Reduktion der Einnahmefrequenz von Medikamenten zur Akuttherapie, in deren Folge es zu einem Rückgang des analgetikainduzierten Dauerkopfschmerzes sowie anderer Nebenwirkungen kommt. Als erfolgreich gilt eine Prophylaxe, wenn sie in der Lage ist, die Anfallshäufigkeit um 50\% zu senken (Evers et al. 2008; Deutsche Gesellschaft für Neurologie 2012).

\subsubsection{Medikamentöse Migräneprophylaxe}

Nach den Leitlinien der Deutschen Migräne- und Kopfschmerzgesellschaft und der Deutschen Gesellschaft für Neurologie 2008 (Evers et al. 2008) sowie der Leitlinie der Deutschen Gesellschaft für Neurologie 2012 (Deutsche Gesellschaft für Neurologie 2012) zählen folgende Medikamente zur ersten Wahl der medikamentösen Migräneprophylaxe: Betablocker, wie Propanolol und Metoprolol, der Kalziumantagonist Flunarizin und die Antikonvulsiva Topiramat und Valproinsäure.

Der genaue Mechanismus, der die prophylaktische Wirkung hervorruft, ist bislang noch nicht geklärt. Die zur Prophylaxe eingesetzten Medikamente scheinen die Sensitivität der kortikalen Zellen gegenüber der CSD herabzusetzen (Ayata et al. 2006).

Unter den Betablockern ist die prophylaktische Wirkung für Metoprolol und Propranolol am besten belegt (Holroyd et al. 1991; Diener und Limmroth 1994; Linde und Rossnagel 2004). Auch andere Betablocker, wie Bisoprolol (Wörz et al. 1992; van de Ven et al. 1997), Atenolol (Stensrud und Sjaastad 1980; Forssman et al. 1983; Johannsson et al. 1987) oder Timolol (Stellar et al. 1984; Tfelt-Hansen et al. 1984), werden als wirksam angesehen, sind aber in Studien weniger untersucht. Es wird davon ausgegangen, dass noch weitere Betablocker effektiv sind. Als nicht effektiv gelten hingegen die Betarezeptorenblocker Pindolol (Sjaastad und Stensrud 1972),

Oxprenolol (Ekbom und Zetterman 1977), Acebutolol (Nanda et al. 1978) und Alprenolol (Ekbom 1975).

Die Wirkweise der Betablocker in der Migräneprophylaxe werden in der Blockade des 5HT2-Rezeptors (Feuerstein und Diener 2013) vermutet. Unter der Therapie können Nebenwirkungen wie Bradykardie, Asystolie, Hypotonie, verminderte Durchblutung, Müdigkeit, psychische Störungen, Schwindel, verzögerte Symptome bei Hypoglykämie und 
Bronchospasmus auftreten (Lüllmann et al. 2003; Deutsche Gesellschaft für Neurologie 2012). Insgesamt zeigen Betablocker aber in der Langzeiteinnahme eine relativ gute Verträglichkeit.

Aus der Gruppe der Kalziumkanalblockern ist Flunarizin der Einzige zur Migräneprophylaxe zugelassene (Louis 1981; Diener et al. 2002). Die Wirkweise ist noch unbekannt (Feuerstein und Diener 2013). In vergleichenden Studien mit Propanolol zeigt Flunarizin eine vergleichbare Wirksamkeit. Es wird aber aufgrund des ungünstigeren Nebenwirkungsprofils seltener als die Betablocker eingesetzt (Sørensen et al. 1991). Als Nebenwirkungen werden hier Müdigkeit und Gewichtszunahme beschrieben (Deutsche Gesellschaft für Neurologie 2012; Feuerstein und Diener 2013).

Aus der Medikamentengruppe der Antikonvulsiva sind Topiramat und Valproinsäure nachgewiesen wirksam (Mathew et al. 1995; Klapper 1997; Freitag et al. 2002; Brandes et al. 2004; Chronicle und Mulleners 2004; Diener et al. 2004; Silberstein et al. 2004). Die Wirkweise von Topiramat beruht auf verschiedenen Mechanismen, die eine übermäßige Erregung von Nervenzellen verhindern. Topiramat hemmt die Erregung durch Blockierung der Glutamat-Bindungsstelle am aktivierenden AMPA-Rezeptor und wirkt gleichzeitig durch Bindung an GABA-Rezeptoren inhibierend. Außerdem inaktiviert Topiramat spannungsabhängige Natriumkanäle (Feuerstein 2013). Unerwünschte Arzneimittelwirkungen betreffen häufig Störungen in der Kognition und treten meist in der Eindosierungsphase auf (Láinez et al. 2007)

Studien zur Anwendung von Valproinsäure konnten eine Wirksamkeit bezogen auf ein Reduktion der Anfallshäufigkeit, jedoch nicht in Bezug auf die Schmerzintensität belegen (Klapper 1997; Freitag et al. 2002). Die Wirkung beruht auf einer Erhöhung der Konzentration des hemmenden Neurotransmitters GABA im synaptischen Spalt durch Enzymregulation (Feuerstein 2013). Als Nebenwirkungen gelten Tremor, Verwirrtheit, Haarausfall, Gerinnungsstörungen, Pankreatitis und Hepatotoxizität, zudem besteht ein teratogenes Potential (Feuerstein 2013).

Als weitere Antikonvulsiva können Lamotrigin (Lampl et al. 2005), Levetriacetam (Brighina et al. 2006) sowie Zonisamid eingesetzt werden (Mohammadianinejad et al. 2011).

Mittel der zweiten Wahl sind Amitriptylin, Venlaflaxin, Gabapentin, Naproxen, Acetylsalicylsäure, Magnesium, Vitamin B2 in Kombination mit Magnesium (Deutsche Gesellschaft für Neurologie 2012). Diese Substanzen sind entweder in ihrer prophylaktischen Wirksamkeit den Mitteln der ersten Wahl unterlegen, noch nicht ausreichend in Studien auf ihre prophylaktische Wirksamkeit hin untersucht worden oder werden nur für bestimmte Konstellationen empfohlen. Zudem finden einige der oben genannten Substanzen in der Prophylaxe der Migräne außerhalb ihrer Zulassung Anwendung (off-label use). Es kann zu medikamenteninduzierte Nebenwirkungen kommen (Deutsche Gesellschaft für Neurologie 2012). 
Als weitere Substanz scheint Botulinum-Toxin im Einsatz als Onabotolinumtoxin A in der Prophylaxe der chronischen Migräne wirksam zu sein (Evers et al. 2002; Petri et al. 2009; Diener et al. 2010).

Bei den meisten zur Migräneprophylaxe eingesetzten Medikamenten kann der Therapieerfolg erst nach Wochen beurteilt werden. Dies führt dazu, dass die Patienten oft zunächst die Nebenwirkungen verspüren, bevor die prophylaktische Wirkung einsetzt, wodurch die Compliance herabsetzt werden kann. Die Auswahl des Medikamentes sollte sich deshalb auch individuell nach dem Patienten richten und seine Komorbiditäten mit einbeziehen (Evers et al. 2008; Deutsche Gesellschaft für Neurologie 2012). Die medikamentöse Prophylaxe sollte zunächst langsam eingeschlichen werden und bis zu zwei Monate fortgeführt werden, bevor eine therapeutische Wirksamkeit oder ein Therapieversagen festgestellt werden kann (Evers et al. 2008; Deutsche Gesellschaft für Neurologie 2012). Bei erfolgreicher Behandlung sollte die Substanz frühestens nach sechs Monaten wieder abgesetzt werden. Zur Beurteilung der Wirksamkeit wird empfohlen, während der Einnahme der Prophylaxe ein Kopfschmerztagebuch zu führen, aus welchem Frequenz, sowie Dauer und Stärke der Attacken hervorgehen. Zudem sollten Art und Dosis der Schmerztherapie vermerkt werden. Es wird geraten, das Tagebuch mindestens vier Wochen vor Start der Therapie zu beginnen, um die Erfolg der Prophylaxe objektivieren zu können (Evers et al. 2008).

\subsubsection{Nichtmedikamentöse Migräneprophylaxe}

Nichtmedikamentöse Verfahren zur Migräneprophylaxe umfassen nach den Leitlinien der Deutschen Migräne- und Kopfschmerzgesellschaft und der Deutschen Gesellschaft für Neurologie 2008 (Evers et al. 2008) sowie der Leitlinie der deutschen Gesellschaft für Neurologie 2012 (Deutsche Gesellschaft für Neurologie 2012) Maßnahmen zur Verbesserung der körperlichen Leistungsfähigkeit, das Erlernen und Anwenden von Entspannungsverfahren wie der Muskelrelaxation nach Jakobsen oder anderen Biofeedbackverfahren (Nestoriuc und Martin 2007; Andrasik 2010), Stressbewältigungstraining, kognitiv-verhaltensorientierte Verfahren (Andrasik 2004; Seng und Holroyd 2010) und Akupunktur (Linde et al. 2009). Schlaf und Entspannung während des akuten Anfalls sowie als Prophylaxe das Vermeiden von Triggerfaktoren, kombiniert mit einer ausgeglichenen Lebensweise (Goadsby und Sprenger 2010) und Ausdauersport (Busch und Gaul 2008), werden supportiv empfohlen. Diese Änderungen im Verhaltensbereich sollten vor Beginn der medikamentösen Prophylaxe versucht werden und können eine medikamentöse Prophylaxe sinnvoll ergänzen (Evers et al. 2008; Deutsche Gesellschaft für Neurologie 2012).

\subsection{Neuromodulierende Verfahren zur Migränetherapie}

In dem Bereich neuromodulierender Verfahren werden im Einsatz bei Schmerzerkrankungen invasive (periphere Nervenstimulation, insbesondere die des N. occipitalis major, 
Vagusnervenstimulation, zervikale Rückenmarksstimulation und hypothalamische Tiefenhirnstimulation) von nichtinvasive Verfahren (repetitive transkranielle Magnetstimulation und transkranielle Gleichstromstimulation) unterschieden (Jürgens et al. 2011). Voraussetzung zum Einsatz eines der invasiven neuromodulierenden Verfahren bei Migräne ist neben einem chronischen Verlauf eine zusätzliche Therapieresistenz. Des Weiteren sollten invasive Verfahren nur in spezialisierten Zentren und innerhalb von prospektiven Studien durchgeführt werden (Jürgens et al. 2011; Deutsche Gesellschaft für Neurologie 2012). Bisher konnte eine eingeschränkte Wirksamkeit der Stimulation des N. occipitalis major bei chronischer Migräne nachgewiesen werden (Saper et al. 2011).

\subsubsection{Transkranielle Magnetstimulation (TMS)}

Bei der TMS wird durch eine Magnetspule, welche tangential über den Schädel gehalten wird, ein zeitlich veränderliches Magnetfeld erzeugt. Hierdurch wird im Gewebe ein paralleler Stromfluss produziert, welcher eine elektrische Stimulation der Neurone bewirken kann (Siebner und Ziemann 2007; Rüsseler 2009). Die Stärke des elektrischen Feldes nimmt exponentiell mit der Entfernung der Spule ab und hängt zudem von der Frequenz und Dauer der magnetischen Impulse sowie dem Aufbau der Spule ab (Siebner und Ziemann 2007; Rüsseler 2009; Ziemann 2013). Bei einer überschwelligen Depolarisation wird hierdurch ein Aktionspotential generiert (Siebner und Ziemann 2007). Diese durch TMS induzierten Aktionspotentiale breiten sich über die Synapsen aus und bewirken an den Zielneuronen entweder exzitatorische oder inhibitorische postsynaptische Potentiale (Ziemann 2013). Als Reizantwort können je nach Stimulationsort Phosphene, Skotome, Veränderungen der Sprache und Muskelzuckungen generiert werden (Epstein et al. 1996; Amassian et al. 1998; Mottaghy et al. 1999; Hallett 2000; Merabet et al. 2003; Hallett 2007; Wassermann und Zimmermann 2012). Neben den akuten Effekten können Stimulationen mit repetitiver TMS auch langanhaltende Änderungen der Erregbarkeit bewirken, die am ehesten auf Langzeitpotenzierung und Langzeitdepression beruhen (Ziemann 2007).

Eine Unterform der TMS ist die repetitive transkranielle Magnetstimulation (rTMS). Im Gegensatz zur Einzelstimulation werden hier Magnetpulse in einem kurzen Zeitabstand abgegeben. Je nach Stimulationsfrequenz können unterschiedliche Effekte erzielt werden. So kann eine niederfrequente rTMS (Reizfrequenz $\leq 1 \mathrm{~Hz}$ ) ein Absenken, eine hochfrequente rTMS $(5-25 \mathrm{~Hz})$ hingegen ein Anheben der kortikalen Erregbarkeit bewirken (Maeda et al. 2000; Wassermann und Lisanby 2001; Ziemann 2013).

Aufgrund ihrer Wirkweise kann TMS in der experimentellen Hirnforschung zur Untersuchung des Nervensystems und im begrenzten Umfang auch in der neurophysiologischen Funktionsdiagnostik sowie mittlerweile auch zu therapeutischen Zwecken eingesetzt werden. In der Annahme, dass TMS das Ausbreiten einer CSD verhindern kann, scheint die therapeutische Anwendung von TMS besonders bei Migräne mit Aura denkbar (Lipton und Pearlman 2010). 


\subsubsection{Therapeutischer und klinischer Einsatz von TMS bei Patienten mit chronischer und episodischer Migräne im Rahmen von Studien}

Einzelpuls-TMS konnte bereits erfolgreich bei Patienten mit Migräne angewendet werden (Clarke et al. 2006; Mohammad et al. 2006; Lipton et al. 2010; Bhola et al. 2015; Schoenen et al. 2016).

Clarke setzte Einzelpuls-TMS als Akuttherapie während des Anfalls ein und konnte damit eine deutliche Schmerzreduktion sowie anhaltende Schmerzfreiheit bewirken (Clarke et al. 2006).

In einer multizentrischen, placebokontrollierten Studie, in der sich Patienten im Anfall selber stimulierten, konnte durch TMS eine Schmerzreduktion erzielt werden (Lipton et al. 2010). In einer ähnlich aufgebauten Studie ließen sich auch positive Ergebnisse darstellen (Mohammad et al. 2006).

Bhola et al. untersuchten Patienten im Rahmen eines Telefoninterviews nicht placebokontrolliert nach Eigenanwendung von TMS okzipital als Akuttherapie (Bhola et al. 2015). Auch hier zeigte sich die TMS Behandlung vorteilhaft.

Die Studien weisen jedoch teilweise methodische Mängel auf, so dass vor einer therapeutischen Anwendung zunächst die Effektivität von Einzelpuls-TMS wissenschaftlich weiter erforscht werden muss (Deutsche Gesellschaft für Neurologie 2012; Schoenen et al. 2016).

Studien zur repetitiver-TMS (rTMS) als prophylaktische Therapie zeigen bisher indifferente Ergebnisse (Brighina et al. 2002; Brighina et al. 2004; Teepker et al. 2010; Misra et al. 2012).

In der Studie von Brighina et al. erhielten die Patienten mit chronischer Migräne eine hochfrequente (die kortikale Erregbarkeit erhöhende) rTMS (>1Hz) (Machado et al. 2013) Behandlung über dem linken dorsolateralen präfrontalen Kortex (DLPFC). Es konnte eine signifikante Abnahme der Attackenfrequenz sowie eine Reduktion der Schwere des Kopfschmerzes und der Einnahme Akutschmerzmedikation nachgewiesen werden (Brighina et al. 2004).

In einem ähnlichen Studienaufbau wendeten Conforto und Mitarbeiter randomisiert hochfrequente rTMS über dem linken DLPFC an. Hier zeigte sich jedoch in der Stimulationsgruppe eine signifikant erhöhte Anzahl an Tagen mit Kopfschmerzen (Conforto et al. 2014). In der Studie von Teepker et al. erhielten Migränepatienten randomisiert niedrigfrequente (inhibitorische wirkende) rTMS $(\leq 1 \mathrm{~Hz})$ (Machado et al. 2013) über dem Vertex ohne das eine signifikante Reduktion der Migräneattacken in der Stimulationsgruppe nachgewiesen werden konnte (Teepker et al. 2010) .

Misra et al. untersuchte in einer Open-Label-Studie die Anwendung von hochfrequenter rTMS über dem linken Frontallappen (Misra et al. 2012) und in einer weiteren randomisierten und placebokontrollierten Studie hochfrequenter rTMS über M1 (Misra et al. 2013). Misra 
und Kollegen konnten in beiden Studien eine Abnahme der Häufigkeit der Kopfschmerzen nachweisen.

Kalita et al. führten eine Untersuchung mit hochfrequenter rTMS bei Patienten mit Mi-gräne über dem links frontalen Kortex durch. Die Patienten erhielten entweder dreimalig eine rTMS Stimulation oder nur einmalig eine rTMS Stimulation und zweimalig Placebo. Die Kopfschmerzfrequenz konnte gesenkt werden, wobei es keinen signifikanten Unterschied in den beiden Stimulationsprotokollen gab (Kalita et al. 2016).

In einer weiteren Studie wurde inhibitorisches Quadripuls-rTMS über dem visuellen Kortex angewendet. Nachfolgend konnte eine signifikante Abnahme der Kopfschmerzdauer, der Kopfschmerzintensität und des Medikamentenverbrauches verzeichnet werden (Sasso d'Elia et al. 2013).

Die Evidenz für eine prophylaktische Anwendung von rTMS ist aktuell nicht ausreichend, so dass auch nach Angaben der Leitlinie der deutschen Gesellschaft für Neurologie (Deutsche Gesellschaft für Neurologie 2012) bisher keine Empfehlung zur Therapie ausgesprochen werden kann.

\subsubsection{Transkranielle Gleichstromstimulation}

Mit der transkraniellen Gleichstromstimulation (tDCS) steht ein relativ einfaches, nebenwirkungsarmes und kostengünstiges Verfahren zur Verfügung, um die zerebrale Erregbarkeit zu modulieren. Ein großer Vorteil der tDCS ist, dass das Verfahren bei geringer Stromstärke von den Patienten und Probanden kaum wahrgenommen wird und aufgrund dessen in Studien auch eine Placebostimulation erfolgreich durchgeführt werden kann (Nitsche und Paulus 2007).

\subsubsection{Technische Grundlagen}

Über zwei auf der Kopfhaut angebrachte Elektroden wird mittels einer Gleichspannungsquelle ein schwacher Gleichstrom appliziert. Üblicherweise werden dabei leitende Gummielektroden oder Elektroden aus Metall verwendet, welche in einem der Elektrode angepassten Schwamm mit Kontaktmedium (Kochsalz oder leitfähiges Gel) auf die Kopfhaut angebracht werden (Woods et al. 2016).

Bei der anodalen tDCS wird die Anode über der zu stimulierenden Region und die Kathode über der Referenzregion platziert. Bei kathodaler tDCS werden die Elektroden genau umgekehrt angebracht. Dabei hat die Anordnung der Elektroden zueinander einen wesentlichen Einfluss auf den Stromfluss. Zudem wirkt die tDCS auch im Bereich des Austrittsortes, so dass auch im Bereich der Referenzelektrode eine Neuromodulation stattfindet (Bikson et al. 2010). Aufgrund dessen wird der Lokalisation der Referenzelektrode eine wichtige Bedeutung beigemessen (Paulus 2014). 


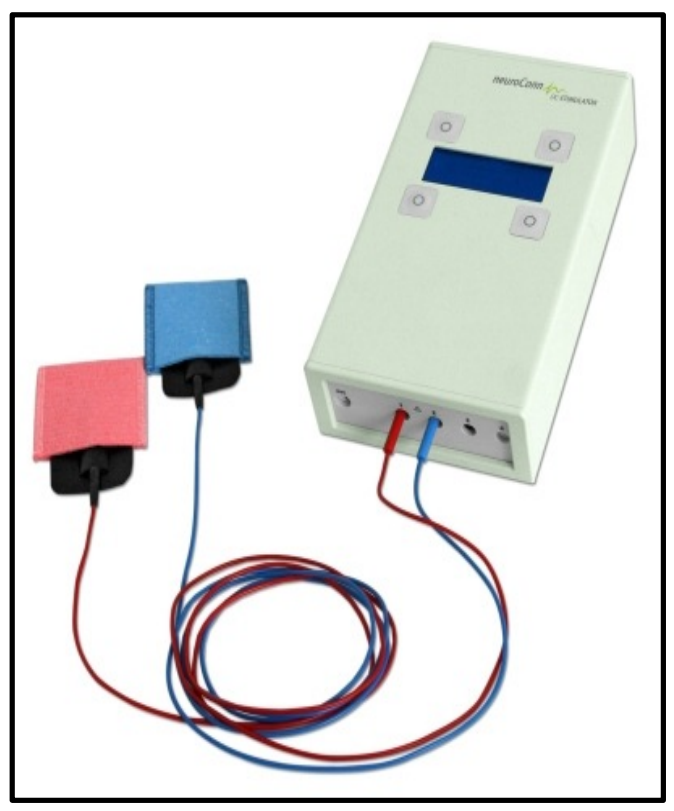

Abbildung 2: Gleichstromstimulator mit Kathode und Anode (neuroConn DC-STIMULATOR). (neurocaregroup.com; die Verwendung erfolgt mit freundlicher Genehmigung von neuroCare Group $\mathrm{GmbH}$.)

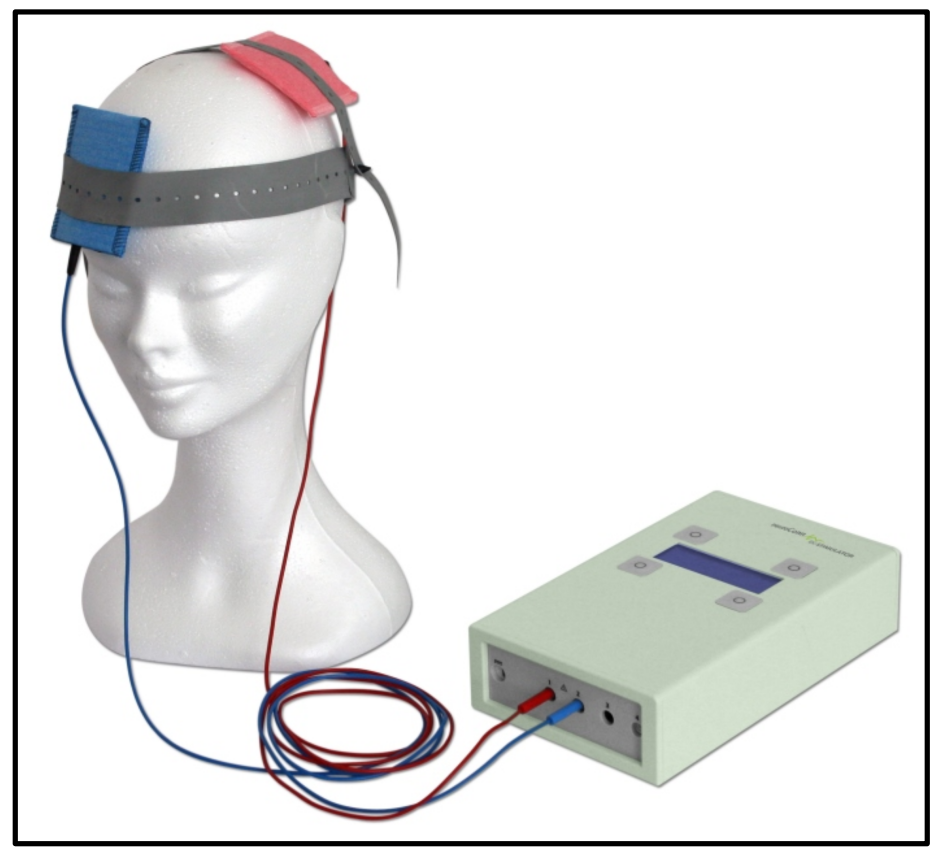

Abbildung 3: Gleichstrom-Stimulator (neuroConn DC-STIMULATOR) mit am Dummy positionierter Kathode und Anode. rocaregroup.com; die Verwendung erfolgt mit freundlicher Genehmigung von neuroCare Group GmbH.)

Bei Menschen erfolgt die Gleichstromstimulation nicht invasiv. Anders als im Tierversuch liegen die Elektroden beim Menschen nicht direkt dem Kortex auf, sondern sind auf der Kopfhaut angebracht. Der verabreichte Strom muss dementsprechend mehrere Schichten durchdringen (Kopfhaut, Schädelkalotte, Dura), wodurch es zu einer ca. 50\% Abschwächung der Stromdichte kommt (Nitsche und Paulus 2007). Neueste Untersuchungen von Buzsaki 
an Kadavern zeigte sogar, dass bis zu 90\% des Stroms vor Erreichen den Kortex abgeleitet werden (Underwood 2016).

\subsubsection{Funktionsweise tDCS}

Durch die Applikation eines kontinuierlichen Gleichstroms von 1-2 mA über einige Minuten über einem bestimmten Kortexareal kann die regionale kortikale Erregbarkeit und neuronale Aktivität verändert werden. Abhängig von der Dauer, Intensität und Polarität können die Effekte Minuten bis Stunden nach der Stimulation andauern (Nitsche und Paulus 2007).

tDCS führt in Abhängigkeit von der Stromrichtung zu einer Verschiebung des Ruhemembranpotentials, ohne jedoch selber direkt ein Aktionspotential auszulösen (Purpura und McMurtry 1965; Nitsche et al. 2008).

Die entsprechenden Auswirkungen auf kortikaler Ebene wurden in tierexperimentellen Studien genauer untersucht. So konnte an Katzen nachgewiesen werden, dass anodale (oberflächenpositive) Gleichstromstimulation durch Depolarisierung zu einer Erregbarkeitssteigerung der Neurone führt, kathodale (oberflächennegative) Stimulation hingegen über eine Hyperpolarisation zu gegenteiligen Effekten führt (Bindman et al. 1964; Nitsche und Paulus 2007).

Anhand verschiedener Studien konnte gezeigt werden, dass sich die Effekte bei einer Stimulationsdauer von weniger als 5 Minuten rasch zurückbilden. Bei zunehmender Dauer und Intensität halten die Effekte hingegen bis zu einigen Stunden nach Beendigung der tDCS an (Bindman et al. 1964; Gartside 1968).

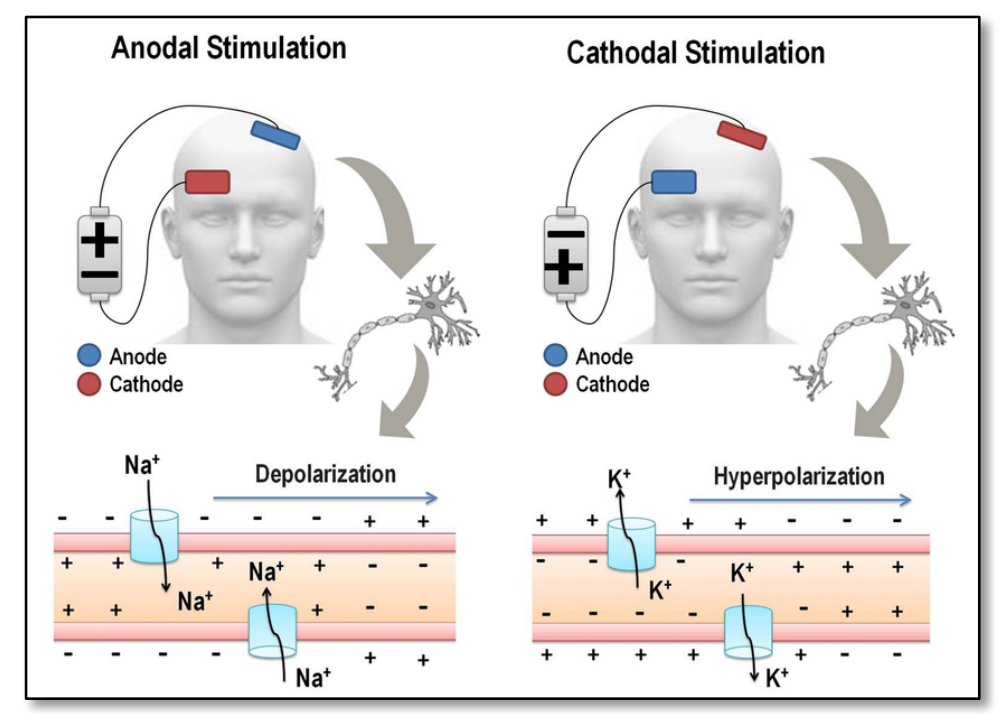

Abbildung 4: Schematische Darstellung der anodalen und kathodalen Stimulation poll Rozisky et al. 2015; die Verwendung erfolgt mit freundlicher Genehmigung von Nova Science Publishers Inc.) 
In Studien am menschlichen Gehirn, welche im Wesentlichen am primären Motorkortex durchgeführt wurden, konnten mittels TMS-Reizen und der Aufzeichnung der hierdurch ausgelösten motorisch evozierten Potentiale (MEP) die durch tDCS erzeugten Exzitabilitätsveränderungen einfach nachgewiesen werden (Nitsche und Paulus 2007). Übereinstimmend mit den Ergebnissen aus den Tierexperimenten konnten Nitsche und Paulus zeigen, dass anodale Stimulation die Erregbarkeit des motorischen Kortex erhöht und kathodale diese vermindert (Nitsche und Paulus 2000). Weiterhin konnten Nitsche und Paulus darlegen, dass auch am primär motorischen Kortex die Wirkungsdauer mit der Dauer und Intensität der tDCS zunimmt (Nitsche und Paulus 2000; Nitsche und Paulus 2001; Nitsche et al. 2003a; Nitsche et al. 2008). Eine höhere Stromdichte (Stromstärke pro Elektrodenfläche) führt zu einer stärkeren Änderung des Ruhemembranpotentials und es werden tiefer gelegene Nervenzellen erreicht (Creutzfeldt et al. 1962; Nitsche und Paulus 2000). Weitere Studien konnten zeigen, dass tDCS nicht nur in der Lage ist, in dem Bereich der Stimulation eine veränderte Membranpolarität zu bewirken, sondern auch in tiefer gelegenen Regionen, welche mit dem stimulierten Areal verbunden sind (Dasilva et al. 2012; Bolzoni et al. 2013b; Bolzoni et al. 2013a; Ripoll Rozisky et al. 2015). Während die akuten Effekte durch eine Verschiebung des Ruhemembranpotentials erklärt werden können, scheinen die Nacheffekte durch Veränderung der neuronalen Verschaltung hervorgerufen zu werden (Ripoll Rozisky et al. 2015). Eine Erklärung für die nachhaltigen Veränderungen der synaptischen Signalübertragung könnte unter anderem die Regulation von Neurotransmittern und die Modellierung sowie Veränderung insbesondere von NMDA-Rezeptoren im Sinne einer Langzeitpotenzierungen (LTP) bzw. einer Langzeitdepression (LTD) sein (Nitsche und Paulus 2007).

Bei der Langzeitpotenzierung spielen der NMDA- und der AMPA-Rezeptor, welche beide Glutamat-Rezeptoren sind, eine zentrale Rolle. Bei Bindung von Glutamat an den AMPARezeptor der postsynaptischen Zelle strömt Natrium in die Zelle, wodurch es zu einer Depolarisation der Zelle kommt. Der NMDA-Rezeptorkanal ist jedoch durch ein MagnesiumIon blockiert, welches erst durch die Depolarisation freigesetzt wird. Durch Freisetzung des Magnesium-Ions fließen nun auch Calcium-Ionen in die Zelle, wodurch es zur Aktivierung von Proteinkinasen kommt. Diese Aktivierung führt bei wiederholter Erregung der Zelle zur Bildung und dem Einbau weiterer Glutamat-Rezeptoren. Es resultiert eine vergrößerte synaptischen Kontaktfläche, wodurch es zu einer Langzeitpotenzierung kommt. Bei niederschwelliger Stimulation werden Enzyme aktiviert, die zur Reduktion der Glutamat-Rezeptoren führen. Die nachfolgende Abnahme der synaptischen Übertragung führt zur Langzeitdepression (Mulkey et al. 1993; Ripoll Rozisky et al. 2015). 


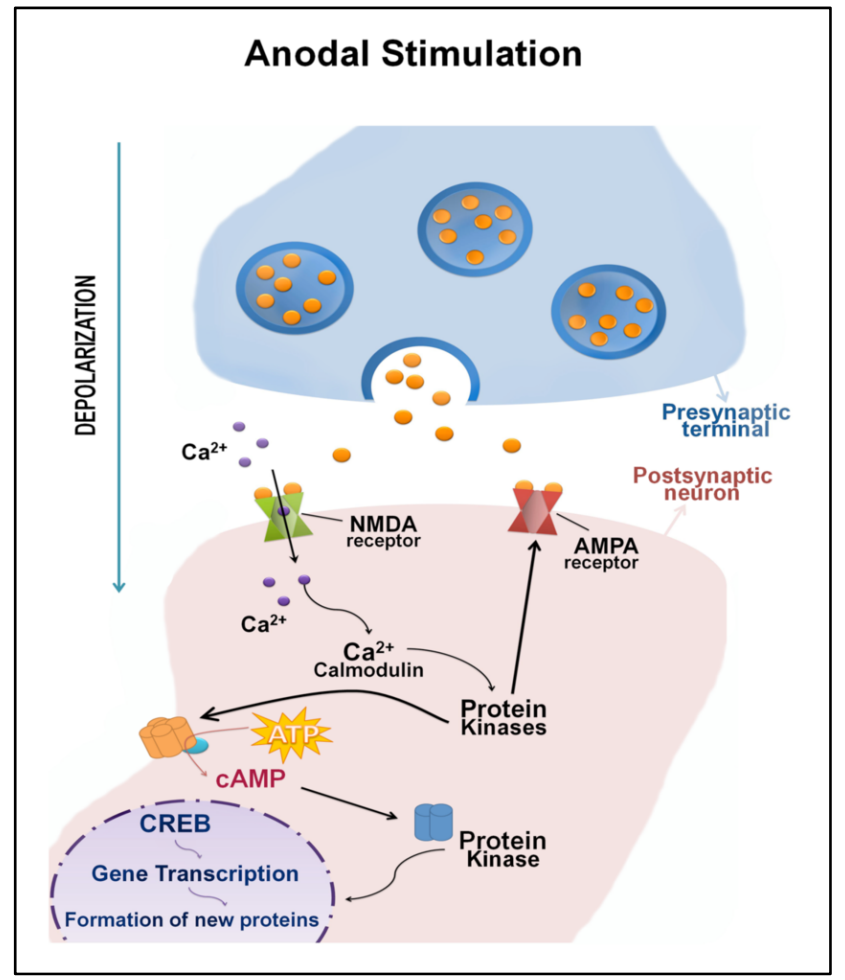

\section{Abbildung 5: Mechanismus der Langzeitpotenzierung durch anodale tDCS}

(Ripoll Rozisky et al. 2015; die Verwendung erfolgt mit freundlicher Genehmigung von Nova Science Publishers Inc.)

Auch die langanhaltenden Nacheffekte durch anodale und kathodale tDCS scheinen NMDA-Rezeptor abhängig (Liebetanz 2002; Nitsche et al. 2004b). So konnte Liebetanz zeigen, dass der NMDA-Rezeptor-Antagonist Dextromethorphan die Nacheffekte von sowohl kathodaler als auch anodaler Stimulation unterbinden kann (Liebetanz 2002). Nitsche konnte nachweisen, dass der Einsatz von D-Cycloserine einem partiellem NMDA-Rezeptor-Agonisten die Nacheffekte von anodaler tDCS verlängern kann, wohingegen kathodale Nacheffekte unbeeinflusst blieben (möglicherweise kann die durch kathodale tDCS reduzierte NMDA-Rezeptor-Aktivität nicht durch einen partiellen Agonisten aufgehoben werden) (Nitsche et al. 2004b). Rohan et al. untersuchten an Ratten, in welcher Weise tDCS einen LTP-ähnlichen Effekt auf die synaptische Plastizität des Hippocampus hat (Rohan et al. 2015). Rohan und Mitarbeiter konnten durch 30 min anodaler tDCS einen anhaltenden Effekt auf die synaptische Plastizität nachweisen, welcher zudem dosisabhängig unter 0,25 mA ausgeprägter war als mit 0,1 mA. Auch in dieser Studie konnten NMDA-Antagonisten die Veränderung der synaptischen Plastizität verhindern (Rohan et al. 2015). Auch andere Studien zeigten, dass lang andauernde schwache tDCS durch intrazelluläre Anreicherung von cAMP und Kalzium molekulare Mechanismen impliziert, die einer LTP gleichen (Hattori et al. 1990; Moriwaki 1991; Islam et al. 1995; Nitsche und Paulus 2007). Entsprechend wird bei kathodaler tDCS von einer verminderter Bahnung ausgegangen (Nitsche und Paulus 2007). 
Die anhaltende Steigerung der Erregbarkeit nach anodaler Gleichstromstimulation ist demnach wahrscheinlich am ehesten die Folge einer LTP, welche durch eine gesteigerte Spontanentladungsrate und durch die Depolarisierung ausgelöst wird (Nitsche und Paulus 2007).

Wie auch in den Studien am primären Motorkortex konnten Studien am visuellen Kortex mittels Messung von Phosphenschwellen mit TMS nach okzipitaler tDCS bidirektionale Konditionierungseffekte nachweisen (Antal et al. 2004).

Verglichen mit den Untersuchungen am Motorkortex waren die Nacheffekte nach tDCS über dem visuellen Kortex kürzer nachweisbar (Antal et al. 2011). Dies ist möglicherweise auf den deutlich komplexeren Aufbau und Verschaltung des visuellen Systems zurückzuführen (Antal et al. 2011). In den Studien von Kraft und Olma konnte nach anodaler tDCS über V1 eine Verbesserung der visuellen Wahrnehmung detektiert werden, nach kathodaler tDCS jedoch nicht (Kraft et al. 2010; Olma et al. 2011). Eine weitere Studie zum perzeptuellen Lernen zeigte nach kathodaler oder anodaler tDCS keinen signifikanten Effekt (Fertonani et al. 2011). In einer anderen Studie stellten Pirulli et al. dar, dass der Zeitpunkt und das Stimulationsprotokoll von anodaler tDCS über V1 einen wesentlichen Einfluss auf das Ergebnis haben. So konnte die Arbeitsgruppe zeigen, dass der Einsatz von anodaler tDCS vor der Aufgabenstellung einen Effekt hatte, jedoch nicht, wenn der Einsatz während des Testes erfolgte (Pirulli et al. 2013). Peters et al. konnten jedoch in einer Studie zeigen, in der anodale, kathodale und Placebostimulation über V1 durchgeführt wurde, dass anodale tDCS den Konsolidierungsvorgang des visuellen Lernens unterdrückt (Peters et al. 2013). Eine weitere Arbeitsgruppe legte dar, dass anodale tDCS über V1 über einen Zeitraum von vier Tagen das visuelle perzeptuelle Lernen verbessern und die kortikale Erregbarkeit steigern kann. Kathodale tDCS hatte in der gleichen Untersuchung keinen signifikanten Effekt auf das Lernen und die Erregbarkeit des visuellen Kortex (Sczesny-Kaiser et al. 2016).

In der Zusammenfassung zeigt sich, dass die Ergebnisse über den Einfluss von tDCS auf das Lernverhalten nicht kongruent sind und durch weitere Studien reevaluiert werden müssen.

\subsubsection{Therapeutische Anwendung von tDCS}

Durch den Nachweis fokale kortikale Erregbarkeitsänderungen zu induzieren, stellt die tDCS ein viel versprechendes neues Verfahren zur Beeinflussung von Neuroplastizität beim Menschen dar. Aufgrund dessen ist neben der neurowissenschaftlichen Anwendung auch eine therapeutische Anwendung denkbar. Der Ansatz tDCS zur therapeutischen Anwendung zu nutzen, erfolgten bereits 1964 bei Patienten mit Depressionen (Costain et al. 1964) und wurde in den letzten Jahren wieder aufgegriffen. So sprechen einige Phase-II-Studien zur Anwendung von tDCS bei Schmerzpatienten für die therapeutische Wirksamkeit. Fregni et al. (Fregni et al. 2006b) führten eine placebokontrollierte Studie durch, welche die Wirksamkeit der tDCS zur Schmerzreduktion bei Patienten mit zentralen Schmerzen nach Verletzungen des Rückenmarks untersuchte. Das Ergebnis der Studie zeigte gegenüber der 
Placebogruppe eine signifikante Schmerzreduktion der Gruppe, welche eine 5-tägige anodale Stimulation des motorischen Kortex mit $2 \mathrm{~mA}$ über 20 Minuten erhalten hatte. In Forschungsarbeiten bei Patienten mit Fibromyalgie konnte (Fregni et al. 2006a; Marlow et al. 2013; Bhola et al. 2015; Fagerlund et al. 2015) eine signifikante Reduktion des Schmerzes mittels anodaler tDCS nachgewiesen werden. Auch bei Patienten mit chronischen, myofaszialen und Phantomschmerzen konnten eine vorübergehende Besserung der Schmerzsymptomatik durch anodale tDCS nachgewiesen werden (Schoenen et al. 1986; Antal et al. 2010; Mori et al. 2010; Sakrajai et al. 2014; Bolognini et al. 2015).

Denkbar ist eine Anwendung im therapeutischen Bereich vor allem bei Erkrankungen, die mit einer pathologisch veränderten kortikalen neuronalen Aktivität im Zusammenhang stehen, zu denen auch die Migräne gehört.

\subsubsection{Prophylaktischer Einsatz von tDCS bei Migräne}

Zum Einsatz von tDCS bei Migräne erfolgten bisher wenige Studien mit verschiedenen Stimulationsprotokollen und inhomogenen Ergebnissen.

Liebetanz konnte in einem Experiment mit anästhesierten Ratten nachweisen, dass anodale tDCS eine Zunahme der Ausbreitungsgeschwindigkeit der CSD bewirkt, kathodale und Placebostimulation jedoch nicht. Demzufolge könnte eine anodale tDCS möglicherweise die Auslösung einer Migräneattacke begünstigen (Liebetanz et al. 2006)

Viganò et al. führten eine kleine, nicht-placebo kontrollierte Studie durch, in der anodale tDCS über dem visuellen Kortex appliziert wurde und konnte so eine signifikante Reduktion der Frequenz, der Migräne-assoziierten Tage und gesamt Stundenanzahl sowie eine Reduktion der Akutmedikation nachweisen (Viganò et al. 2013). Dasilva et al. stimulierten über dem primär motorischen Kortex mit anodaler tDCS und konnte im Gegensatz zur Placebostimulation in der Nachbeobachtungszeit nach Beendigung der Stimulation, jedoch nicht während der Stimulation, eine signifikante Verringerung der Schmerzintensität und Dauer der Migräneattacke vorweisen (Dasilva et al. 2012).

Auvichayapat hingegen führte über der primär-motorischen Rinde (M1) eine anodale tDCS durch und konnte mit anodaler Stimulation im Vergleich zur Placebogruppe eine statistisch signifikante Reduktion der Attackenfrequenz, Schmerzintensität und der Einnahme von Schmerzmedikation feststellen (Auvichayapat et al. 2012).

Bei Rocha et al. erhielten die Patienten eine prophylaktische kathodale tDCS über dem visuellen Kortex. Es konnte nur in der Verumgruppe eine signifikante Reduktion der Dauer der einzelnen Anfällen und der Schmerzmitteleinnahme verglichen mit Ausgangsniveau nachgewiesen werden (Rocha et al. 2014).

In einer weitere Studie erfolgte selektiert an Patientinnen mit menstrueller Migräne kathodale tDCS über dem visuellen Kortex, wodurch eine signifikante Reduktion der Anzahl der 
Migräneattacken, verglichen mit dem Ausgangsniveau aber nicht verglichen mit der Placebostimulation, erzielt werden konnte (Wickmann et al. 2015).

\subsubsection{Sicherheit der Methode}

tDCS ist nach bisheriger Studienlage ein sicheres und nebenwirkungsarmes Verfahren (Nitsche et al. 2003b; Nitsche et al. 2003c; Iyer et al. 2005; Fertonani et al. 2015; Bikson et al. 2016), wodurch die tDCS bei Beachtung der Richtlinien hinsichtlich Kontraindikationen und Stimulationsparameter ein gut verträgliche Verfahren im therapeutischen Bereich ist. Es gibt keinen Hinweis auf eine durch tDCS induzierte persistierende neuronale Schädigung (Nitsche et al. 2003b; Nitsche et al. 2003c; Bikson et al. 2016; Antal et al. 2017).

Als Nebenwirkung werden Müdigkeit, vorübergehende Kopfschmerzen, Lichtblitze, Übelkeit sowie elektrisch induzierte lokale Muskelkontraktionen während der Stimulation angegeben. Relativ häufig kommt es zu eine Reizung der Kopfhaut, welche sich in Form von Kribbeln, Jucken und Brennen äußern kann (Poreisz et al. 2007; Fertonani et al. 2015). Dabei nehmen die Hautirritationen mit der Stromstärke und auch der Elektrodengröße zu (Turi et al. 2014; Fertonani et al. 2015).

Bisher konnten keine Schäden am Nervengewebe nachgewiesen werden, jedoch kann die Stimulation im Bereich der Kontaktstelle von Elektrode zur Haut zu einer Verbrennungen des Hautgewebes führen (Nitsche et al. 2003b; Nitsche et al. 2004a; Antal et al. 2017). Dies kann jedoch durch die Verwendung von salzwassergetränkten Schwammelektroden verhindert werden (Nitsche et al. 2003c). Durch eine entsprechend konzentrierte NaCl-Lösung kann der Hautwiderstand verkleinert werden, wodurch es zu weniger Hautirritationen kommt (Dundas et al. 2007; Fertonani et al. 2015). In einem Fallbericht ist eine Hautläsion im Bereich der Elektrode nach einer tDCS mit $2 \mathrm{~mA}$ beschrieben (Palm et al. 2008; Brunoni et al. 2011).

In einem Review von Brunoni et al., in welchem 172 Studien eingeschlossen wurden, waren die am häufigsten beschrieben Nebenwirkungen im Vergleich von akitiver tDCS versus Placebobehandlung Juckreiz (39,3\% versus 32,9\%), Prickeln (22,2\% versus 18,3\%), Kopfschmerz (14,8\% versus 16,2\%), Brennen (8,7\% versus $10 \%)$ und Unwohlsein (10,4 versus 13,4 \%). Es traten keine gravierenden Nebenwirkungen auf. Laut Brunoni et al. gibt es in der Literatur nur einen Fallbericht, in dem eine schwerwiegende Nebenwirkung in Form einer respiratorischen Insuffizienz nach tDCS auftrat (Redfearn et al. 1964; Brunoni et al. 2011). Die Autoren bemängeln jedoch, dass bisher nicht immer eine systematische Abfrage von Begleiterscheinungen bei Studien mit Anwendung von tDCS erfolgte und empfehlen dies für die Zukunft, um garantieren zu können, dass tDCS in unterschiedlicher Anwendung bei verschiedenen Patienten ein sicheres Verfahren ist (Brunoni et al. 2011).

Auch in einer Untersuchung an Kindern und Jugendlichen konnten keine ernsthaften Nebenwirkungen nach tDCS mit $1 \mathrm{~mA}$ über M1 festgestellt werden. Nach tDCS wurde zudem eine EEG-Aufzeichnung vorgenommen, in welcher sich keine epilepsietypischen Potentiale 
nachweisen ließen (Moliadze et al. 2015). In einem Review zeigten sich nach tDCS bei Kindern und Jugendlichen nur selten und wenn vorhanden, gering ausgeprägte Nebenwirkungen wie Hautrötung, Kribbeln, Jucken und Brennen (Krishnan et al. 2015)

\subsection{Ziele der vorliegenden Studie}

Die zur Migräneprophylaxe eingesetzten Medikamente haben eine begrenzte Wirkung und sind nicht immer verträglich, so dass komplementäre Therapien von großem Interesse sind. Insbesondere bei Patienten, die unzureichend auf Medikamente ansprechen, Medikamente schlecht vertragen oder eine für die medikamentöse Behandlung problematische Komorbidität zeigen, wäre ein alternatives Verfahren vorteilhaft.

Seit einiger Zeit gewinnen nichtinvasive Stimulationsverfahren wie TMS und tDCS bei der Behandlung von Schmerz und Kopfschmerzen an Bedeutung (Lipton et al. 2010; Lipton und Pearlman 2010). TMS wurde zunächst aufgrund der Annahme eingesetzt, dass es das Auftreten von CSD verhindern könne. Unterschiedliche Studien zeigen, dass nach Applizierung von rTMS über dem visuellen Kortex oder Arealen, welche bei der Schmerzwahrnehmung zu Beginn der Migräneattacke von Bedeutung sind, es bei einem hohen Prozentsatz der Patienten zu einer Reduktion oder sogar Beendigung der Kopfschmerzen kam (Clarke et al. 2006; Mohammad et al. 2006; Lipton und Pearlman 2010).

Als Prophylaxe über dem linken dorsolateralen präfrontalen Kortex (DLPFK) eingesetzte Hochfrequenz-rTMS, welche zwölfmalig an verschiedenen Tagen appliziert wurde, konnte die Attackenhäufigkeit bei chronischen Migränepatienten reduzieren (Brighina et al. 2004). Eine weitere Studie, in der prophylaktisch an fünf hintereinander folgenden Tagen mit Niedrigfrequenz-rTMS $(1 \mathrm{~Hz})$ der Vertex stimuliert wurde, zeigte ebenfalls eine deutliche Reduktion der Migräneattacken (Teepker et al. 2010). In ähnlicher Weise wie TMS und andere nichtinvasive Methoden für die Stimulation des Gehirnes kann tDCS eine reversible umschriebene Veränderung der Erregbarkeit des Gehirns bewirken.

Migränepatienten berichten über visuelle Missempfindungen beim Betrachten von Streifenmustern (Wilkins et al. 1984). Zudem weisen sie im Vergleich zu gesunden Kontrollprobanden Unterschiede in der visuellen Verarbeitung auf kortikaler Ebene in psychophysischen (Wray et al. 1995; Palmer et al. 2000; Shepherd 2001; Antal et al. 2005) und auch elektrophysiologischen Studien auf (Schoenen et al. 2003). Diese Unterschiede zwischen Probanden mit und ohne Migräne, welche in verschiedenen Messungen zur visuellen Wahrnehmung dargelegt werden konnten, deuten auf eine pathologische visuelle Verarbeitung auf kortikaler Ebene hin. Es gibt Hinweise darauf, dass die Erregbarkeit des visuellen Kortex insbesondere im migränefreien Intervall erhöht ist (Aurora et al. 1998; Mulleners et al. 2001b; Huang et al. 2003; Brighina et al. 2004; Antal et al. 2005; Chadaide et al. 2007). Eine Hyperreaktivität besteht bei dem Gehirn von Migränepatienten hauptsächlich in Hinsicht auf wiederholte Stimuli (Coppola et al. 2007). 
Die tDCS kann funktionelle Veränderungen in der Hirnrinde bewirken, wodurch auch die Schmerzwahrnehmung und Schmerzschwelle moduliert werden kann. Somit steht mit der transkraniellen Gleichstromstimulation eine potentielle Alternative zu den herkömmlichen Therapieverfahren zur Verfügung, das auf seine Eignung zur Akuttherapie und prophylaktischen Behandlung der Migräne gegenwärtig überprüft wird.

Ausgehend von der Annahme, dass für die Entstehung der Migräne eine erhöhte neuronale Erregbarkeit des visuellen Kortex mit ursächlich ist, wurde durch die kathodale tDCS eine Methode aufgezeigt, die eine Verminderung kortikaler Erregbarkeit bewirken kann. Dies legt die Vermutung nahe, dass die kathodale tDCS des okzipitalen Kortex durch Absenken der kortikalen Erregbarkeit möglicherweise hilfreich ist, Migräneanfälle mit dazugehörigen Begleitsymptomen in ihrer Intensität und Dauer zu reduzieren und sie so auf diese Weise eine wirksame Behandlungsform in der Migräneprophylaxe darstellen könnte.

Die im Folgenden beschriebene Studie wurden durchgeführt, um zu überprüfen, inwieweit kathodale tDCS placebokontrolliert eine Reduktion der Frequenz, Dauer und Intensität von Migräneattacken bewirken kann. Zudem wurde die Effektivität der Verblindung durch Kombination von kathodaler und Placebostimulation einer der beiden Patientengruppen getestet. 


\section{$2 \quad$ Methode}

Die im Folgenden beschriebenen Untersuchungen wurden zwischen 2006 und 2010 in der Klinik für Klinische Neurophysiologie der Georg-August Universität Göttingen durchgeführt.

\subsection{Probanden}

Die Patientengruppe bestand aus insgesamt 30 Migränepatienten. Die Patienten wurden randomisiert der kathodalen Stimulationsgruppe oder der Placebogruppe zugewiesen. Vier von den 30 Patienten wurden aus der insgesamt 22 Wochen andauernden Studie ausgeschlossen, da diese ihre Kopfschmerztagebücher nicht korrekt führten oder nur inkomplett ausfüllten. Von den resultierenden 26 Teilnehmern waren 23 weiblich (w) und drei männlich (m) und wiesen ein Durchschnittsalter von 44,4 Jahren auf. Die Probanden befanden sich im Alter von 20 bis 53 Jahren. Alle Patienten wurden mittels standardisierten Kopfschmerzfragebogens befragt. Die Diagnose Migräne musste den Kriterien der 2004 International Headache Society (IHS) für Migräne ohne Aura, Migräne mit Aura oder chronischer Migräne entsprechen. Nach den Leitlinien der IHS (siehe 1.3.1) erfüllten 14 Probanden die Kriterien für Migräne mit Aura (12 w, 2 m) und 12 ohne Aura (11 w, 1 m).

Für die Teilnahme an der Studie galten folgende Einschlusskriterien:

- Migräne mit und ohne Aura nach IHS-Kriterien

- Dauer der Migräneerkrankung > ein Jahr

- mindestens zwei Attacken pro Monat

- während der gesamten sechs Monate vor Studienbeginn keine Einnahme einer prophylaktische Migränemedikation

Zum Ausschluss der Studie führten folgende Kriterien:

- Herzschrittmacher

- Metallimplantate im Kopfbereich

- Alter $<18$ oder $>65$ Jahre

- Hinweise auf chronische oder Residuen einer neurologischen Erkrankung in der Vorgeschichte

- intrazerebrale Ischämien in der Vorgeschichte

- Hinweise auf epileptische Anfälle in der Vorgeschichte

- Vorliegen einer gravierenden internistischen oder psychiatrischen Vorerkrankung, insbesondere Schizophrenie oder Manie

- Schwangerschaft oder Stillperiode

- Drogen-, Medikamenten- und/oder Alkoholabhängigkeit

- vorherige Teilnahme an tDCS Untersuchungen 
- Episodische oder chronische Spannungskopfschmerzen bedeuteten keinen Ausschluss aus der Studie. Der Unterschied zwischen Spannungskopfschmerzen und Migräne-Attacken musste im Kopfschmerztagebuch jedoch klar erkennbar sein.

Die Ethikkommission der Georg-August-Universität Göttingen genehmigte die Studie. Alle Personen nahmen freiwillig und informiert an der Studie teil, nachdem sie ihr schriftliches Einverständnis gegeben hatten (Studiennummer: 1/5/03).

\subsection{Studiendesign}

Die Studie wurde in drei Phasen unterteilt:

I. Zunächst erfolgte eine Evaluation des Grundlevels. Vor Beginn der Stimulation führten die Probanden für acht Wochen ein für die Studie entworfenes Migränetagebuch. Mittels dieses Tagebuches wurde die Häufigkeit und Dauer der Migräneattacken, die Intensität, Beginn und Dauer des Schmerzes, Begleiterscheinungen, sowie Wirkstoff und Dosierung der Medikamenteneinnahme festgehalten.

II. In der darauf anschließenden Phase erfolgte eine sechswöchige Behandlung der Probanden für drei Tage pro Woche. Die Behandlung bestand in einer 15-minütigen Placebo- oder kathodalen tDCS. Während der ersten drei Wochen erhielten alle Probanden eine Placebostimulation, in der zweiten Hälfte wurde bei einer Hälfte der Patienten weiterhin nur eine Placebostimulation durchgeführt; bei der anderen Hälfte hingegen kathodale Stimulation angewendet. Die Randomisierung der Teilnehmer erfolgte durch die Reihenfolge des Eintritts in die Studie. Jeder zweite Patient erhielt demnach die kathodale Stimulation. Auch während des Zeitraums der Stimulation führten die Patienten weiterhin das Migränetagebuch.

III. In den acht auf die Stimulation folgenden Wochen wurde das Tagebuch zur Nachbeobachtung fortgeführt. Eine Synopse des Studienaufbaus ist in der folgenden Abbildung zu finden. 


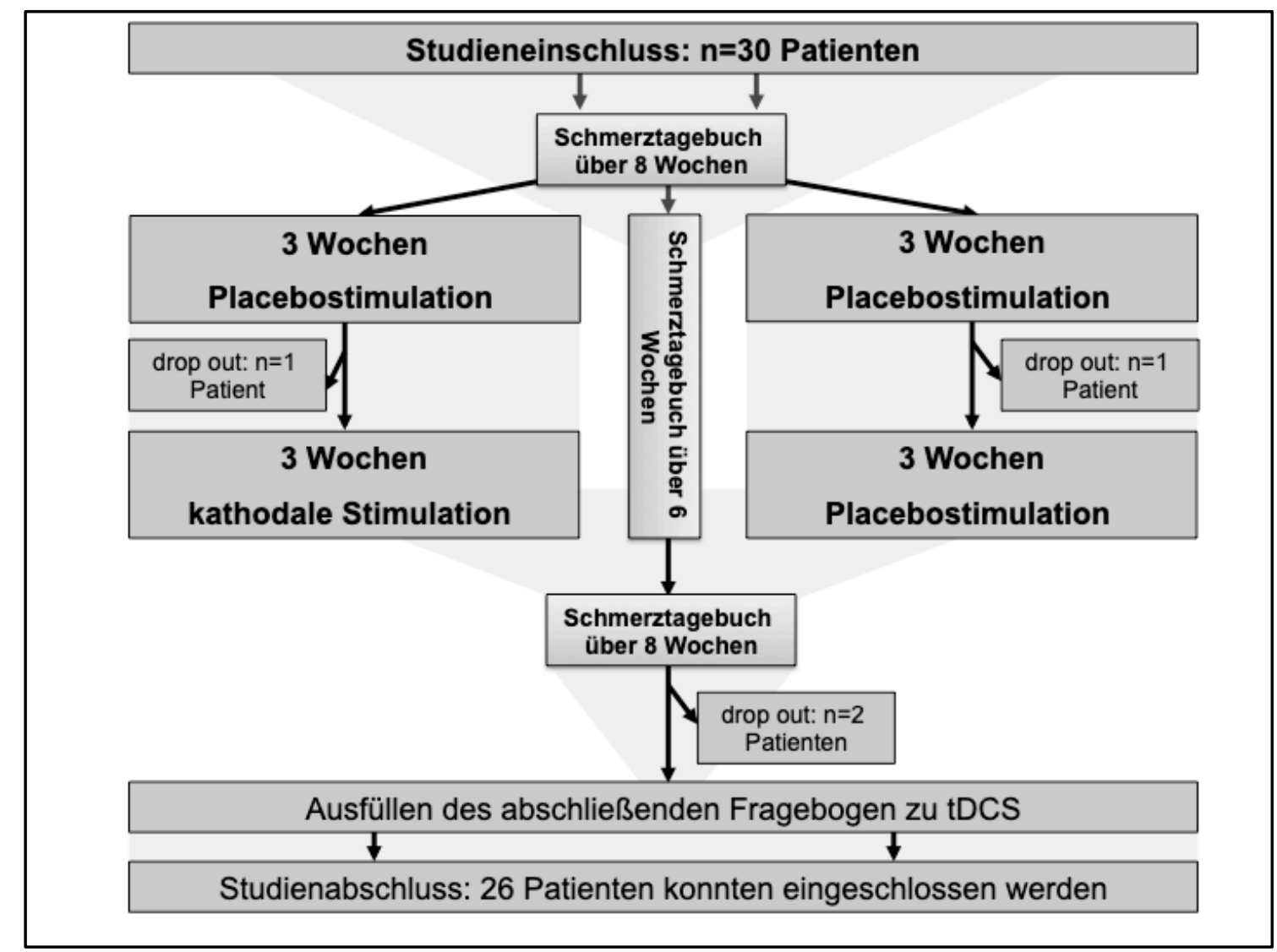

Abbildung 6: Studienaufbau mit Anzahl an Patienten, die im Studienverlauf ausgeschlossen wurden und Zeitpunkt des Ausschlusses im Verlauf der Studie. 26 Patienten haben die Studie beendet.

\subsection{Transcranial direct current stimulation (tDCS)}

Während der Stimulation saßen die Patienten in einem bequemen Liegesitz mit Kopflehne. Die Gleichstromstimulation wurde mittels zweier Oberflächenelektroden durchgeführt, welche sich beide jeweils in einem in $0,9 \% \mathrm{NaCl}$ getränkten Schwamm von $5 \times 7 \mathrm{~cm}$ befanden. Die kathodale Elektrode wurde über dem okzipitalen Kortex, die Referenzelektrode über dem Vertex durch zwei elastische EEG-Bänder angebracht. Die Stimulation erfolgte über einen speziell entwickelten, batteriebetriebenen Gleichstromstimulator (NeuroConn, Ilmenau, Germany). Die kathodale tDCS wurde mit einer Stromstärke von $1 \mathrm{~mA}$ für 15 Minuten verabreicht. Beide Elektroden wurden von den Probanden in Form von leichtem Kribbeln zu Beginn der Stimulation wahrgenommen.

Die Positionierung der Elektroden für die Placebostimulation erfolgte analog zu der kathodalen Stimulation, jedoch schaltete sich der Stimulator nach $30 \mathrm{~s}$ automatisch aus. Aufgrund dessen fühlten die Probanden zu Beginn der Placebostimulation auch ein leichtes Kribbeln, ohne die kathodale tDCS während der restlichen Stimulationsdauer zu erhalten. Die Probanden wurden hinsichtlich der Stimulationsart verblindet. 


\subsubsection{Nebenwirkungen der tDCS}

Nach Protokoll der Studie wurde die Gleichstromstimulation dreimal die Woche für jeweils 15 Minuten durchgeführt. Da über die durch tDCS erzeugten Nebenwirkungen wenig bekannt ist, füllten die Patienten diesbezüglich nach Beendigung der Stimulation einen Fragebogen (Poreisz et al. 2007) aus. Der Fragebogen enthielt eine Beurteilungsscala, welche das Auftreten und den Schweregrad von Kopfschmerzen, Schwierigkeiten in der Konzentration, Stimmungsveränderungen, visuelle Wahrnehmungsstörungen, Ermüdung sowie unangenehme Empfindungen wie Schmerz, Kribbeln, Jucken und Brennen unter den Elektroden während und nach der tDCS dokumentierte.

\subsection{Statistische Analyse}

Für die statistische Auswertung der Studie wurden die Zielkriterien

- Anzahl der Migräneattacken

- Anzahl der mit Migräne belegten Tage

- Dauer der Schmerzen in Stunden

- Schmerzintensität

für die jeweiligen Phasen (I.) vor der Stimulation, (II.) während tDCS und (III.) nach tDCS anhand der Mittelwerte für jeden Probanden berechnet. Die kleine Anzahl an Probanden in beiden Gruppen und die nicht normale Distribution vieler der dokumentierten Parameter in der Studie erlauben nicht die Anwendung einer ANOVA (analysis of variance). Stattdessen wurden anhand des Mann-Whitney-U-Tests die Unterschiede zwischen der Placebogruppe und der Gruppe mit Stimulation berechnet. Um die Effekte, die durch die Behandlung in der Studie herbeigeführt wurden zu vergleichen, wurde entsprechend der Wilcoxon-SignedRank-Test durchgeführt. Das Signifikanzlevel wurde bei $\mathrm{p} \leq 0,05$ gesetzt.

Das Auftreten von durch tDCS verursachte Nebenwirkungen wurde mit einem binären System aufgefasst (nein $=0, j a=1$ ), der Schweregrad des jeweiligen Nebeneffektes wurde hingegen anhand einer numerischen Analogskala (NAS) von 1-5 (1 für eine sehr schwache und 5 für eine starke Intensität) eingestuft. Das Auftreten der Nebenwirkungen sowie deren Schweregrad wurden getrennt - während der tDCS und nach Beendigung der tDCS - berechnet (Poreisz et al. 2007). 


\section{Ergebnisse}

Von 30 in die Studie eingeschlossenen Patienten mussten aufgrund persönlicher Gründe $(n=2)$ sowie von unvollständigen Fragebögen $(n=2)$ insgesamt vier Patienten $(n=4)$ ausgeschlossen werden.

Vor der Stimulation gab es keinen signifikanten Unterschied zwischen den beiden Gruppen bezogen auf die Anzahl der Migräneattacken ( $p=0,77)$; die Anzahl der Migräne-assoziierten Tage $(p=0,14)$, die Dauer der Migräneattacke $(p=0,44)$ und die Intensität des Schmerzes während der Attacke $(\mathrm{p}=0,88)$.

Tabelle 1: Ausgangssituation nach Anamneseerhebung in beiden Stimulationsgruppen

\begin{tabular}{|l|c|c|}
\hline & Kathodal & Placebo \\
\hline Weiblich mit Aura & 5 & 7 \\
\hline Weiblich ohne Aura & 7 & 4 \\
\hline Männlich mit Aura & 1 & 1 \\
\hline Männlich ohne Aura & - & $32,3(12,3)$ \\
\hline Durchschnittsalter (SD) & $33,2(10,4)$ & 6 \\
\hline Schmerzlokalisation & 11 & 7 \\
Einseitig & 2 & $12,0(8,9)$ \\
Beidseitig & $15,3(12,1)$ & $27,7(24,5)$ \\
\hline Durchschnittsdauer in Jahren (SD) & $28,9(24,0)$ & 9 \\
\hline Durschnittanzahl Attacken / Jahr & & 2 \\
\hline Familienanamnese & 6 & 3 \\
Positiv & 5 & 5 \\
Migräne Medikation & 3 & 3 \\
ASA (Aspirin $\mathbb{B})$ & 5 & 4 \\
Triptane & 1 & Codein \\
Ibuprofen & 0 & \\
Paracetamol & Pestwurz & \\
Keine & & \\
Andere & & \\
\hline
\end{tabular}

\subsection{Frequenz der Migräneattacken}

Innerhalb der Stimulationsgruppe und der Placebogruppe war die Anzahl der Migräneattacken nicht signifikant verringert, wenn das Grundlevel mit dem Ergebnis nach Stimulation verglichen wird (kathodal: $\mathrm{p}=0,38$; Placebo: $\mathrm{p}=0,2)$. Werden die Effekte zwischen der Placebogruppe und der Stimulationsgruppe nach Stimulation verglichen, lässt sich keine Signifikanz feststellen $(\mathrm{p}=0,88)$. 


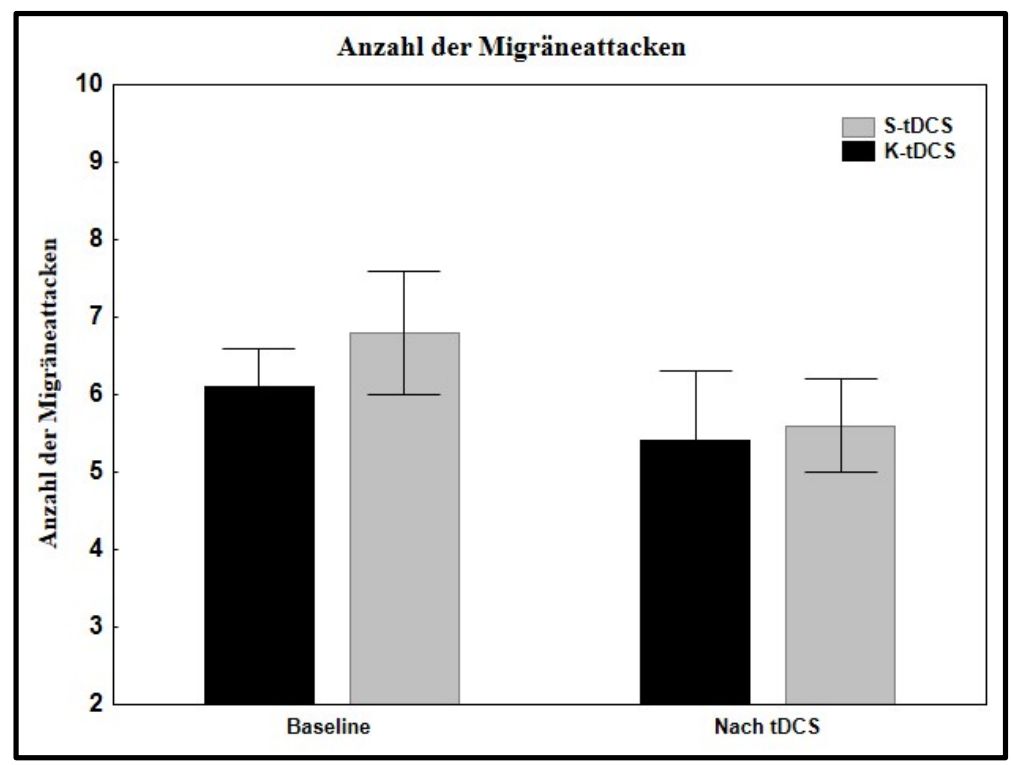

Abbildung 7: Durchschnittliche Anzahl der Migräneattacken im Intervall acht Wochen vor und acht Wochen nach tDCS für die kathodale (K-tDCS) und Placebogruppe (S-tDCS)

\subsection{Anzahl der Tage mit Migräne}

In der Gruppe mit Stimulation konnte nach Stimulation eine signifikante Reduktion der Anzahl der Tage mit Migräne (von 16,2 +/- 2,4 Tagen auf 9,31 +/- 1,5 Tage; $\mathrm{p}=0,004$ ) nachgewiesen werden, in der Placebogruppe hingegen nicht (von 12,8 +/- 2,77 Tagen auf 11,0 +/- 3,5 Tage; $\mathrm{p}=0,17)$. Allerdings konnte im direkten Vergleich der Migräne-assoziierten Tage nach Stimulation zwischen der Placebogruppe und der Stimulationsgruppe kein signifikanter Unterschied nachgewiesen werden $(\mathrm{p}=0,61)$.

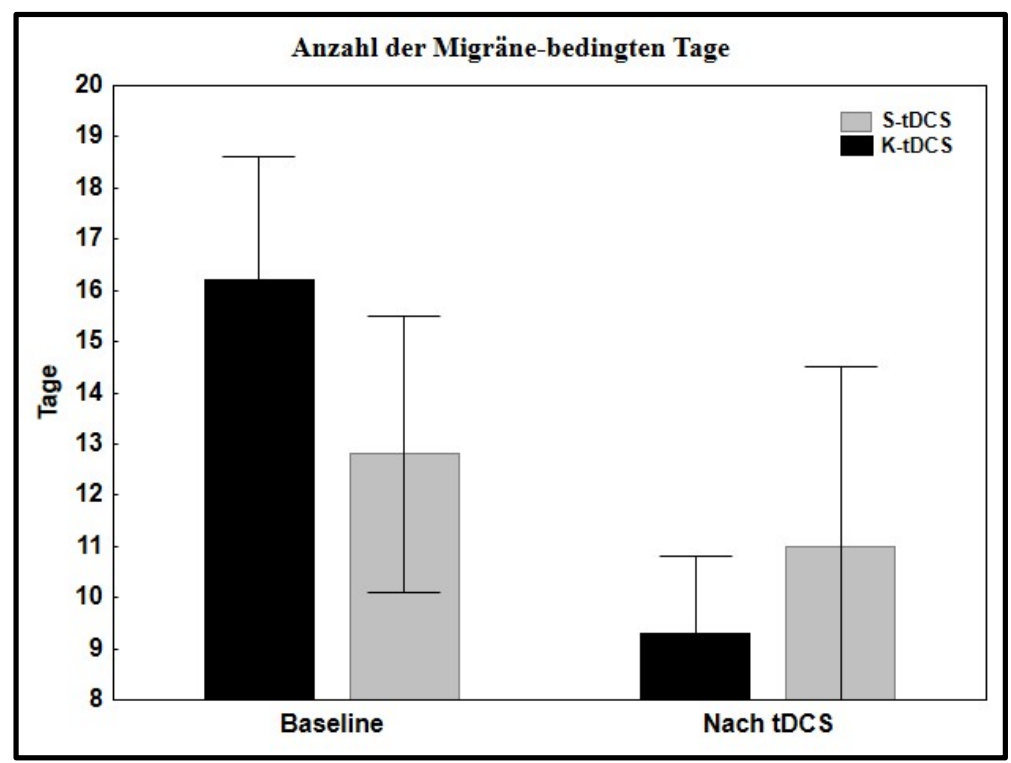

Abbildung 8: Anzahl der Migräne-assoziierten Tage 8 Wochen vor und 8 Wochen nach tDCS für die kathodale (K-tDCS) und Placebogruppe (S-tDCS) 


\subsection{Dauer der Migräneattacke}

Es gab eine signifikante Reduktion der Durchschnittsdauer der Migräneattacken nach Stimulation in der Stimulationsgruppe (von 8,95 +/-1,6 Stunden auf 7,2 +/- 1,6 Stunden; $\mathrm{p}=0,05$ ), aber nicht in der Placebogruppe (von 9,9 +/-1,5 Stunden auf 9,4 +/-1,8 Stunden; $\mathrm{p}=0,55)$. Im direkten Vergleich der durchschnittlichen Dauer der Attacken nach der Stimulation zwischen der Stimulationsgruppe und der Placebogruppe konnte jedoch kein signifikanter Unterschied festgestellt werden $(\mathrm{p}=0,38)$.

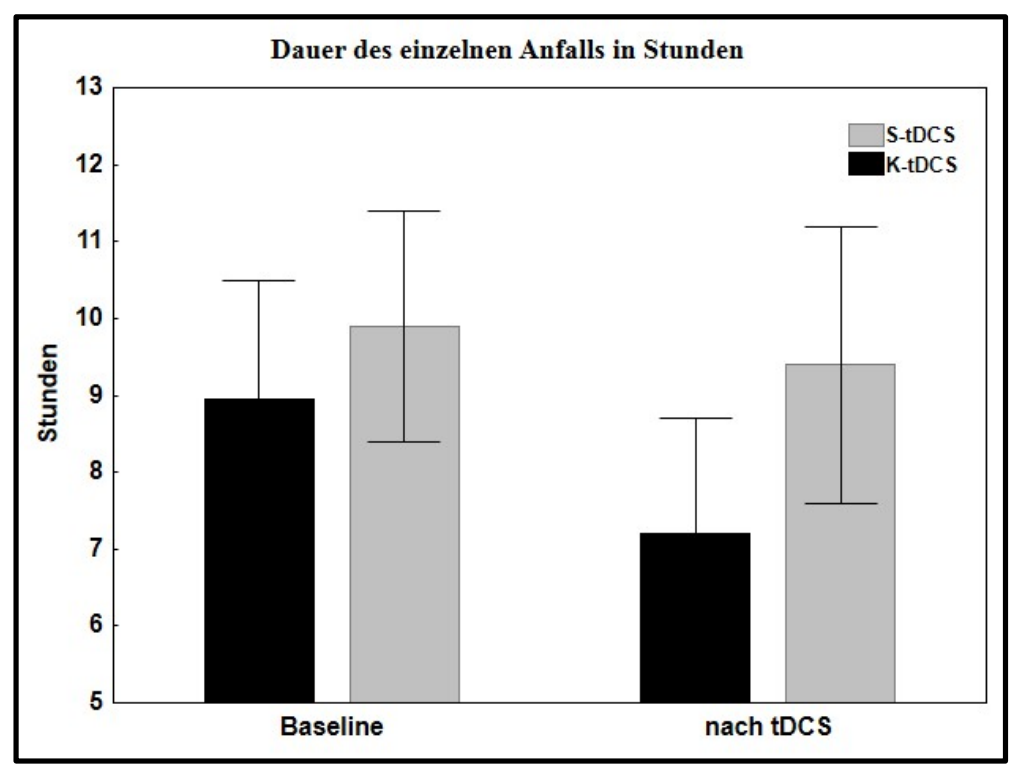

Abbildung 9: Durchschnittliche Dauer der einzelnen Migräneattacken in Stunden acht Wochen vor und acht Wochen nach tDCS in der kathodalen (K-tDCS) und Placebogruppe (S-tDCS)

\subsection{Schmerzintensität}

In der Stimulationsgruppe verringerte sich die Schmerzintensität bezogen auf das Grundlevel im Vergleich zu den Werten nach tDCS signifikant (von 1,99 +/- 0,1 auf 1,54 +/- 0,2; $\mathrm{p}=0,02$ ). In der Placebogruppe gab es keine signifikante Änderung (von 1,88 + /- 0,2 auf 1,92 $+/-0,2 ; \mathrm{p}=0,7)$. Der Vergleich der Schmerzintensität nach Stimulation zwischen der Placebo- und der Stimulationsgruppe ergab einen signifikanten Unterschied $(p=0,05)$. 


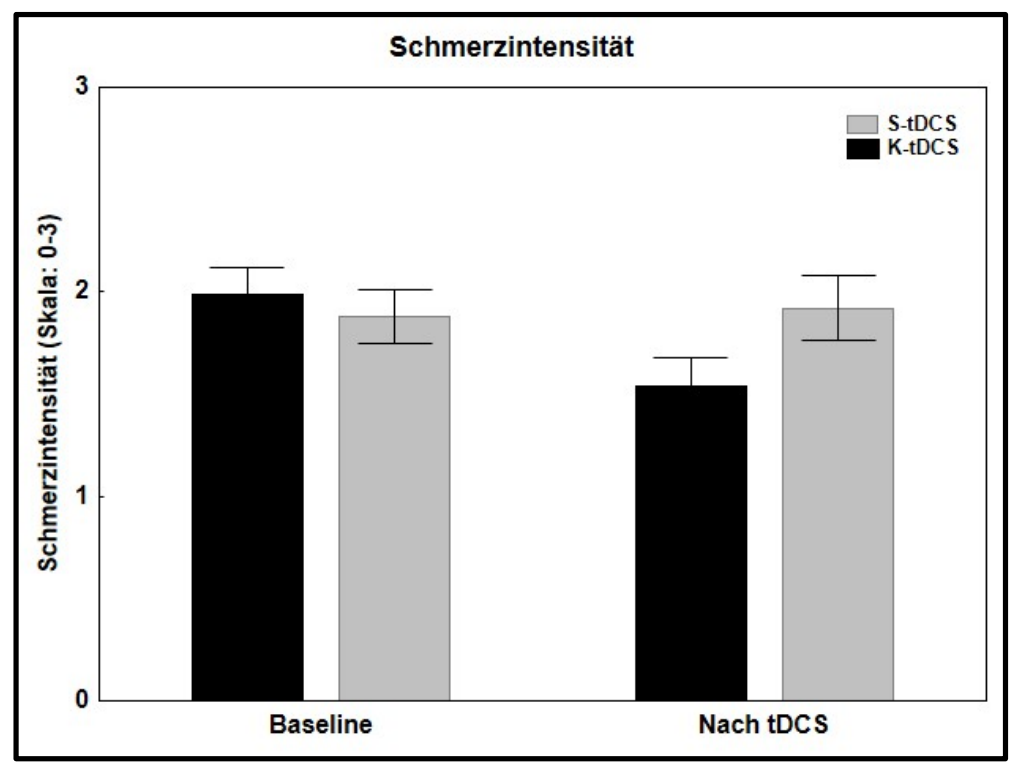

Abbildung 10: Durchschnittliche Schmerzintensität auf einer Schmerzskala von null (kein Schmerz) bis drei (maximale Schmerzstärke) acht Wochen vor und acht Wochen nach tDCS für die kathodale (K-tDCS) und Placebogruppe (S-tDCS)

\subsection{Vergleich von MA- und MO-Patienten}

Bedauerlicherweise war aufgrund der geringen Anzahl von Patienten in beiden Gruppen ein statistischer Vergleich von MA- und MO-Patienten nicht möglich. Die Frequenz, Dauer und Intensität der Migräneattacken sind in Tabelle 2 zusammengefasst. Die Anzahl der Migräneassoziierten Tage reduzierte sich in der kathodalen Gruppe unabhängig von dem Vorhandensein einer Aura. Die Schmerzintensität verringerte sich jedoch in der Gruppe der MAPatienten in einem höheren Maße.

Tabelle 2: Durchschnittliche Anzahl der Migräneattacken und Anzahl der Tage mit Migräne sowie Dauer in Stunden und Schmerzintensität (0 bis 3) im Vergleich der Patienten mit (MA) und ohne (MO) visuelle Aura

\begin{tabular}{|c|c|c|c|c|c|c|c|c|}
\hline & \multicolumn{4}{|c|}{$\overline{\text { MA }}$} & \multicolumn{4}{|c|}{ MO } \\
\hline & \multicolumn{2}{|c|}{ kathodal $(n=6)$} & \multicolumn{2}{|c|}{ Placebo $(n=8)$} & \multicolumn{2}{|c|}{ kathodal $(n=7)$} & \multicolumn{2}{|c|}{ Placebo $(n=5)$} \\
\hline & vorher & nachher & vorher & nachher & vorher & nachher & vorher & nachher \\
\hline Anzahl der Attacken & $\begin{array}{l}6,0 \pm \\
0,7\end{array}$ & $\begin{array}{l}5,0 \pm \\
1,6\end{array}$ & $\begin{array}{l}6,7 \pm \\
1,9\end{array}$ & $6,0 \pm 0,9$ & $\begin{array}{ll}6,14 \quad \pm \\
0,8\end{array}$ & $5,7 \pm 0,9$ & $6,8 \pm 1,1$ & $5,0 \pm 1,1$ \\
\hline $\begin{array}{l}\text { Anzahl der Tage mit } \\
\text { Migräne }\end{array}$ & $\begin{array}{ll}16,5 & \pm \\
3,2 & \end{array}$ & $9,3 \pm 2,4$ & $\begin{array}{ll}15,0 & \pm \\
4,2 & \end{array}$ & $13,6 \pm 5,6$ & $\begin{array}{l}16,0 \quad \pm \\
3,7\end{array}$ & $9,2 \pm 2,2$ & $9,2 \pm 1,4$ & $6,8 \pm 1,2$ \\
\hline Dauer in Stunden (h) & $\begin{array}{ll}10,8 \quad \pm \\
2,9\end{array}$ & $9,6 \pm 2,9$ & $\begin{array}{ll}10,1 & \pm \\
2,2 & \end{array}$ & $9,0 \pm 2,8$ & $7,4 \pm 1,6$ & $5,1 \pm 1,2$ & $9,6 \pm 2,0$ & $10,0 \pm 1,9$ \\
\hline Schmerzintensität & $2,2 \pm 0,2$ & $1,4 \pm 0,3$ & $2,0 \pm 0,1$ & $1,9 \pm 0,2$ & $1,8 \pm 0,1$ & $1,7 \pm 0,1$ & $1,7 \pm 0,3$ & $1,9 \pm 0,4$ \\
\hline
\end{tabular}




\subsection{Nebenwirkungen durch tDCS}

Insgesamt wurde der Fragebogen von 28 Patienten, davon 14 aus der Placebogruppe und 14 aus der Stimulationsgruppe, ausgefüllt, unabhängig davon, ob sie die Stimulation frühzeitig beendet hatten oder eine medikamentöse Therapie während oder nach der Stimulation benötigten. Tabellen 3 und 4 fassen die Nebenwirkungen während und nach Stimulation zusammen. Ein leichtes Kribbeln während der kathodalen tDCS war mit 78,1\% der am häufigsten angegebene Nebeneffekt. Aber auch bei Placebostimulation wurde von 71,0\% ein leichtes Kribbeln bemerkt. Häufig wurde auch ein moderates Jucken empfunden (21,3\% während kathodaler Stimulation versus 28,4\% während der Placebostimulation).

Müdigkeit während der Stimulation wurde von 14,2\% der Probanden und interessanterweise in 28,4\% während der Placebostimulation empfunden. 7,1\% der Probanden der Stimulationsgruppe und sogar 42,6\% der Placebogruppe fühlten sich nach der Stimulation müde. Kopfschmerzen traten in 21,3\% nach kathodaler Stimulation und in 35,5\% nach Placebostimulation auf.

Nach Beendigung der Stimulationsperiode wurden die Probanden befragt, ob sie ihrer Meinung nach eine oder verschiedene Arten von Stimulation erhalten hätten. 21,3\% der Placebogruppe gaben an, verschiedene Arten von Stimulation erhalten zu haben, wohingegen die Probanden aus der kathodalen Gruppe zu 100\% glaubten, nur eine Stimulationsart erhalten zu haben.

Tabelle 3: Prozentsatz an Patienten und Art der wahrgenommenen Nebenwirkungen während der tDCS. MI: durchschnittliche Intensität (mean intensity) auf einer Skala von 0 bis 5 .

\begin{tabular}{|c|c|c|c|c|c|c|}
\hline & \multicolumn{2}{|c|}{ Schmerz } & \multicolumn{2}{|c|}{ Kribbelgefühl } & \multicolumn{2}{|c|}{ Jucken } \\
\hline & $\%$ & MI & $\%$ & MI & $\%$ & MI \\
\hline Placebo & 14,2 & 1,5 & 71,0 & 1,6 & 28,4 & 1,7 \\
\hline \multirow[t]{3}{*}{ kathodal } & 14,2 & 1,0 & 78,1 & 1,0 & 21,3 & 1,0 \\
\hline & \multicolumn{2}{|c|}{ Brennen } & \multicolumn{2}{|c|}{ Müdigkeit } & \multicolumn{2}{|c|}{ Nervosität } \\
\hline & $\%$ & MI & $\%$ & MI & $\%$ & MI \\
\hline Placebo & 14,2 & 2,0 & 28,4 & 2,7 & 7,1 & 1,0 \\
\hline \multirow[t]{3}{*}{ kathodal } & 14,2 & 1,0 & 14,2 & 2,0 & 0,0 & 0,0 \\
\hline & \multicolumn{2}{|c|}{ Konzentrationsschwäche } & \multicolumn{2}{|c|}{$\begin{array}{l}\text { Veränderung der visuellen Wahr- } \\
\text { nehmung }\end{array}$} & \multicolumn{2}{|c|}{ Kopfschmerz } \\
\hline & $\%$ & MI & $\%$ & MI & $\%$ & MI \\
\hline Placebo & 0,0 & & 0,0 & 0 & 21,3 & 1,3 \\
\hline \multirow[t]{3}{*}{ kathodal } & 0,0 & & 7,1 & 1,0 & 0,0 & 0,0 \\
\hline & \multicolumn{2}{|c|}{ unangenehmes Gefühl } & & & & \\
\hline & $\%$ & MI & & & & \\
\hline Placebo & 7,1 & 1,0 & & & & \\
\hline kathodal & 0,0 & 0,0 & & & & \\
\hline
\end{tabular}


Tabelle 4: Prozentsatz an Patienten und Art der wahrgenommenen Nebenwirkungen nach der

tDCS. MI: durchschnittliche Intensität (mean intensity) auf einer Skala von 0 bis 5

\begin{tabular}{|c|c|c|c|c|c|c|}
\hline & \multicolumn{2}{|c|}{ Schmerz } & \multicolumn{2}{|c|}{ Kribbelgefühl } & \multicolumn{2}{|c|}{ Jucken } \\
\hline & $\%$ & MI & $\%$ & MI & $\%$ & MI \\
\hline Placebo & 7,1 & 1,0 & 7,1 & 1,0 & 14,2 & 1,0 \\
\hline \multirow[t]{3}{*}{ kathodal } & 0,0 & 0,0 & 7,1 & 1,0 & 0,0 & 0,0 \\
\hline & \multicolumn{2}{|c|}{ Brennen } & \multicolumn{2}{|c|}{ Müdigkeit } & \multicolumn{2}{|c|}{ Nervosität } \\
\hline & $\%$ & MI & $\%$ & MI & $\%$ & MI \\
\hline Placebo & 14,2 & 1,0 & 42,6 & 2,2 & 0,0 & 0,0 \\
\hline \multirow[t]{3}{*}{ kathodal } & 0,0 & 0,0 & 7,1 & 2,0 & 0,0 & 0,0 \\
\hline & \multicolumn{2}{|c|}{ Konzentrationsschwäche } & \multicolumn{2}{|c|}{$\begin{array}{l}\text { Veränderung der visuellen } \\
\text { Wahrnehmung }\end{array}$} & \multicolumn{2}{|c|}{ Kopfschmerz } \\
\hline & $\%$ & MI & $\%$ & MI & $\%$ & MI \\
\hline Placebo & 0,0 & & 0,0 & 0,0 & 35,5 & 2,0 \\
\hline kathodal & 0,0 & & 0,0 & 0,0 & 21,3 & 2,3 \\
\hline
\end{tabular}




\section{$4 \quad$ Diskussion}

Die nichtinvasive elektrische Hirnstimulation ist ein Verfahren, welches eine langandauernde Veränderung der neuronalen Erregbarkeit bewirken kann. Aufgrund dessen wurde in verschiedenen Studien versucht, die veränderte kortikale Aktivität bei Migränepatienten mit nichtinvasiver Hirnstimulation zu normalisieren.

Davon ausgehend, dass Migräne mit einer neuronalen Übererregbarkeit zwischen den Attacken assoziiert ist, stellten wir die Hypothese auf, dass eine inhibitorische tDCS über dem Kortexareal V1 eine effektive Migräneprophylaxe sein könnte. Tatsächlich konnten wir, verglichen mit dem Ausgangsniveau, eine moderate und signifikante Reduktion der Dauer der Migräne, der Migräne-assoziierten Tage und Schmerzintensität bei den Probanden mit Verumstimulation beobachten. Bezogen auf die Anzahl der Attacken hingegen, konnte keine evidente Reduktion erhoben werden. Im direkten Vergleich der Placebogruppe und der Gruppe mit Verumstimulation war nur die Schmerzintensität signifikant bei den Patienten nach Stimulation reduziert.

\subsection{Vergleich mit Studien zu tDCS bei Migräne}

In der gegenteiligen Annahme zu unserer Arbeitshypothese gingen Viganò et al. von einem erniedrigtem Präaktivierungsniveau des visuelle Kortex bei Migränepatienten aus und führten eine anodale tDCS über dem visuellen Kortex durch (Viganò et al. 2013). Es wurde bei insgesamt 10 MO-Patienten eine anodale tDCS von $1 \mathrm{~mA}$ über 15 Minuten zweimal pro Woche über insgesamt acht Wochen verabreicht. Trotz anodaler tDCS konnte eine signifikante Reduktion der Frequenz, der Migräne-assoziierten Tage und der gesamten Stundenanzahl sowie eine Reduktion der Akutmedikation erreicht werden. Der Effekt war im zweiten Monat der tDCS-Behandlung sogar noch stärker. Jedoch wurde in dieser Studie nur Verumstimulation angewandt, so dass ein Placeboeffekt nicht auszuschließen ist.

In einer Studie von Auvichayapat wurde bei 37 Migränepatienten (aktive $n=20$, sham n=17) eine prophylaktische Behandlung mit anodaler tDCS von $1 \mathrm{~mA}$ mit einer Dauer von $20 \mathrm{Mi}$ nuten an 20 aufeinander folgenden Tagen über der primär-motorischen Rinde (M1) durchgeführt (Auvichayapat et al. 2012). Es erfolgte ein Follow-up nach vier, acht und 12 Wochen. Es konnte mit anodaler Stimulation im Vergleich beider Gruppen eine statistisch signifikante Reduktion der Attackenfrequenz, Schmerzintensität und der Einnahme von Schmerzmedikation festgestellt werden.

Sowohl die Studie von Viganò als auch die Arbeit von Auvichayapat weisen darauf hin, dass tDCS in der klinischen Anwendung bei Migränepatienten effektiv ist. Da in den beiden oben genannten Studien jedoch von einer anderen Pathogenese ausgegangen und somit eine unterschiedliche Polarität verwendet wurde, ist der Vergleich mit unserer Studie aufgrund der verschiedenen Studiendesigns schwierig. 
In einer Studien von Rocha et al. erhielten Patienten mit Migräne prophylaktische kathodale tDCS über dem visuellen Kortex (Rocha et al. 2014). In dieser Arbeit wurde zunächst im migränefreien Intervall elektrophysiologisch die Erregbarkeit verglichen. Hierfür wurde der Schwellenwert zum Auslösen eines Phosphenes eruiert, welcher mit der niedrigsten Intensität durch TMS ausgelöst werden konnte. Dieser Schwellenwert wird als phosphene threshold (PT) bezeichnet. Es zeigte sich, dass der PT-Mittelwert der Migränepatienten deutlich niedriger als bei den Kontrollprobanden war. Dieses Ergebnis wurde als Übererregbarkeit gewertet, woraufhin im therapeutischen Teil der Studie, bei dem eine 20-minütige kathodale tDCS mit $2 \mathrm{~mA}$, bei 15 der 23 oben genannten Migränepatienten dreimal pro Woche über einen Zeitraum von vier Wochen durchgeführt wurde. Die Patienten wurden randomisiert in eine Versuchs- und Kontrollgruppe unterteilt. Das Kopfschmerztagebuch wurde 30 Tage vor der Behandlung, während der Behandlung und 30 Tage nach der Behandlung geführt. Nachfolgend konnte nur in der Verumgruppe eine signifikante Reduktion der Dauer der einzelnen Anfällen und der Schmerzmitteleinnahme, verglichen mit dem Ausgangsniveau, nachgewiesen werden. Die Anfallshäufigkeit war nicht während der aktiven tDCS, aber anschließend in der Nachkontrolle vermindert. Die Schmerzintensität war nicht signifikant verändert. Im direkten Vergleich der Versuchs- und Kontrollgruppe zeigte sich jedoch in Bezug auf die Anzahl an Migräneattacken, Dauer der einzelnen Anfällen und die Schmerzmitteleinnahme kein signifikanter Unterschied. Die Autoren erklären diese fehlende Signifikanz durch das kleine Patientenkollektiv. Die Phosphene-Schwellenwerte vor und nach tDCS zeigten keine signifikanten Unterschiede. Daraus lässt sich schlussfolgern, dass sich die kortikale Erregbarkeit durch die tDCS anscheinend nicht verändert hat. Die Autoren begründen dies damit, dass möglicherweise die ineffiziente Reizverarbeitung bei Migräne tDCS einen veränderten modulierenden Einfluss auf das Gehirn hat. So konnten bereits in vorangegangenen Studien gezeigt werden, dass sowohl tDCS als auch TMS geringer ausgeprägte bzw. sogar paradoxe Effekte auf die kortikale Erregbarkeit des Gehirns bei Migränepatienten bewirkten (Brighina et al. 2002; Chadaide et al. 2007; Antal et al. 2008).

Verglichen mit der Studie von Rocha et al. liegt bei der von uns durchgeführten Studie ein ähnliches Studiendesign vor. Wie in unserer Studie konnte auch in der Arbeit von Rocha et al. in der Verumgruppe eine signifikante Reduktion der Dauer der einzelnen Anfälle, verglichen mit dem Ausgangsniveau, nachgewiesen werden. Jedoch war in unserer Arbeit die Attackenfrequenz nicht verändert, wohingegen die Schmerzintensität signifikant vermindert war. Der Grund für diese unterschiedlichen Ergebnisse ist nicht eindeutig ersichtlich. Die Anzahl der Patienten ( $\mathrm{n}=15)$ in der Arbeit von Rocha et al. war kleiner als in unserer Studie $(\mathrm{n}=26)$. Möglicherweise war die Anzahl der Patienten in beiden Studien zu klein, um reproduzierbare Ergebnisse aufzuzeigen.

Wickmann et al. führten bei 16 Patientinnen mit menstrueller Migräne eine kathodale tDCS über dem visuellen Kortex über drei Menstruationszyklen durch (Wickmann et al. 2015). Die Stimulation erfolgte doppelt verblindet und mit einer Intensität von $2 \mathrm{~mA}$ über 20 Minuten während fünf aufeinanderfolgenden Tagen vor Beginn der Menstruation. Mittels TMS wurde 
die Schwelle zum Auslösen von Phosphenen gemessen, um entsprechend Veränderungen der Erregbarkeit des visuellen Kortex während der Untersuchung zu verifizieren. Durch die kathodale Stimulation konnte eine signifikante Reduktion der Anzahl der Migräneattacken verglichen mit dem Ausgangsniveau erzielt werden. Im direkten Vergleich der Stimulationsgruppe mit der Placebostimulation ergab sich jedoch kein signifikanter Unterschied. Die Schwelle zum Auslösen von Phosphenen war in der Stimulationsgruppe signifikant niedriger als bei den Patienten mit Placebostimulation, was für eine reduzierte Erregbarkeit des visuellen Kortex durch kathodale Stimulation spricht.

Verglichen mit unserer Studie wurde ein ähnliches Stimulationsprotokoll aber mit höherer Stimulationsstärke gewählt. Wickmann et al. konnten im Gegensatz zu unserer Studie im Vergleich zum Ausgangsniveau eine Reduktion der Attackenfrequenz bewirken. Möglicherweise ist die Stimulation mit $2 \mathrm{~mA}$ effektiver als mit $1 \mathrm{~mA}$.

Zusammengefasst sind sowohl die Stimulationsprotokolle, welche tDCS bei Migräne einsetzten, als auch die Patientenkollektive teilweise unterschiedlich und auch die Ergebnisse nicht eindeutig. In Zukunft sollten weitere Studien die Effektivität von tDCS in der prophylaktischen und auch therapeutischen Anwendung verifizieren.

\subsection{Vergleich mit Studien zu TMS bei Migräne}

Repetitive TMS kann die Erregbarkeit des Gehirns nachhaltig verändern, so bewirkt eine niederfrequente rTMS ein Absenken, eine hochfrequente rTMS hingegen eine Anhebung der kortikalen Erregbarkeit (Maeda et al. 2000; Wassermann und Lisanby 2001). Auch die Studien, welche die Effekte von rTMS in der Migräneprophylaxe untersucht haben (Brighina et al. 2004; Teepker et al. 2010), führten ähnlich zu den Studien mit tDCS konträre rTMSStimulationen durch.

In der Studie von Brighina et al. erhielten die Patienten mit chronischer Migräne eine hochfrequente rTMS-Behandlung über dem linken DLPFC in 12 Sitzungen an alternierenden Tagen (Brighina et al. 2004). Jede rTMS-Sitzung bestand aus 10 Einzelstimulationen mit einer Dauer von zwei Sekunden, separiert durch 30 Sekunden Pause, welche mit einer 20-HzFrequenz und motor threshold (MT) (Schwelle zur Auslösung motorischer Antworten) mit einer Intensität von 90\% verabreicht wurden. Die Autoren dieser Studie berichten von einer signifikanten Reduktion der Kopfschmerzattacken, der Anzahl fehlgeschlagener Medikationseinnahmen und des Kopfschmerzindexes nach Stimulation im Vergleich zur Placebostimulation. In dieser Studie waren nur 11 Patienten eingeschlossen, von denen sechs eine Verumstimulation erhielten.

Auch Conforto et al. verwendeten eine hochfrequente TMS $(10 \mathrm{~Hz})$ bei 18 Patienten über dem linken DLPFC an. Die Stimulationsgruppe hatte jedoch in dieser Studie eine signifikant erhöhte Anzahl an Tagen mit Kopfschmerzen (Conforto et al. 2014). 
In der Studie von Teepker et al. waren 27 Migränepatienten eingeschlossen, von denen 14 Patienten eine Verumstimulation erhielten (Teepker et al. 2010). In dieser Studie wurden die Probanden mit einer niedrigfrequenten rTMS, bestehend aus Stimulationen mit 500 Impulsen mit einer Frequenz von $1 \mathrm{~Hz}$ an fünf aneinander folgenden Tagen, über dem Vertex behandelt. Es konnte eine signifikante Reduktion der Migräneattacken in der Stimulationsgruppe festgestellt werden. Jedoch konnte im Vergleich zwischen der Stimulationsgruppe und der Placebogruppe kein signifikanter Unterschied eruiert werden. Das Gleiche gilt für die Migräne-assoziierten Tage und die Gesamtstundenanzahl mit Migräne. Bezogen auf die Schmerzintensität und den Gebrauch von Analgetika konnte auch kein Effekt festgestellt werden.

In weiteren Studien mit inhomogenen Stimulationsprotokollen zur prophylaktischen Anwendung von TMS zeigten sich tendenziell positive Ergebnisse.

Misra et al. verwendeten prophylaktisch sowohl hochfrequenter rTMS $(10 \mathrm{~Hz})$ über dem linken Frontallappens als auch über M1 durch (Misra et al. 2012; Misra et al. 2013). In beiden Studien konnte eine Abnahme der Häufigkeit der Kopfschmerzen nachgeweisen werden.

Kalita et al. führten auch eine Untersuchung mit hochfrequenter rTMS (10 Hz) bei Patienten mit Migräne über dem links frontalen Kortex durch. In der einen Stimulationsgruppen wurde dreimal eine rTMS-Stimulation durchgeführt, in der anderen Gruppen wurde einmal eine rTMS-Stimulation und zweimal eine Placebobehandlung durchgeführt. Als Ergebnis konnte die Kopfschmerzfrequenz gesenkt werden, wobei es keinen signifikanten Unterschied in den beiden Stimulationsprotokollen gab (Kalita et al. 2016). Nach inhibitorischer QuadripulsrTMS über dem visuellen Kortex konnte eine signifikante Abnahme der Kopfschmerzdauer, der Kopfschmerzintensität und des Medikamentenverbrauches verzeichnet werden (Sasso d'Elia et al. 2013). Zudem hielten die Ergebnisse noch einen Monat nach Behandlung an. Die Untersuchung ist bisher aber nicht durch eine Placebostimulation kontrolliert.

In Anlehnung an die oben genannten Studienergebnisse scheint die Applikation von einzelnen oder nur wenigen Magnetpulsen erfolgreicher in der Behandlung akuter Migräneattacken zu sein.

Clarke et al. behandelten 42 Probanden mit Migräne mit Doppelpuls-TMS fünf Sekunden hintereinander folgend über dem Areal, an dem der Schmerz wahrgenommen wurde, oder über dem Areal des Gehirns, von dem aus sich die Aura zu Beginn der Attacke ausbreitete (Clarke et al. 2006). Nach 20 Minuten konnte eine Besserung bei 69\% der Probanden festgestellt werden. Die durchschnittliche Reduktion der Schmerzintensität betrug 25\%.

Lipton et al. untersuchten eine sehr große Anzahl von Probanden (267 Patienten), in der sTMS als Akuttherapie eingesetzt wurde (Lipton et al. 2010). Es konnte eine deutlich höhere Rate an Schmerzfreiheit nach zwei Stunden in der Verumgruppe mit 39\% im Vergleich zur Placebogruppe mit 22\% erreicht werden. Zudem war die gemessene Schmerzfreiheit in der Verumgruppe signifikant länger. 
Bezogen auf unsere Studie und die nicht signifikanten Ergebnisse zwischen der Stimulationsund Placebogruppe müssen wir in Erwägung ziehen, dass die kathodale Stimulation über V1 möglicherweise die kortikale Übererregbarkeit nicht im gleichen Ausmaß mindern kann, wie in einer Studie mit niedrigfrequenter rTMS beobachtet wurde (Teepker et al. 2010), in welcher eine signifikante Reduktion der Migräneattacken in der Stimulationsgruppe festgestellt werden konnte. Jedoch konnte auch hier im Vergleich zwischen der Stimulationsgruppe und der Placebogruppe kein signifikanter Unterschied eruiert werden. Es zeigte sich jedoch auch in unserer Studie in der Stimulationsgruppe eine klare Tendenz mit einer Reduktion der Attackendauer und Schmerzintensität.

Zusammenfassend kann gesagt werden, dass auch bei den Studien zu TMS bei Migräne die Stimulationsprotokolle teilweise sehr unterschiedlich und auch hier die Ergebnisse nicht eindeutig sind. Ähnlich zu den Ergebnissen bei tDCS konnten in Untersuchungen, in welchen sowohl stimulierende TMS als auch inhibitorische TMS eingesetzt wurde, positive Ergebnisse verzeichnet werden. Aufgrund dessen ist es aktuell nicht eindeutig, inwiefern die Polarisation der Stimulation einen Einfluss auf die Häufigkeit der Migräneattacken, Länge des Migräneanfalls sowie auf die Schmerzstärke und Ausprägung von Begleitsymptomen hat. Aufgrund dessen sollte auch die Anwendung von TMS in der Behandlung von Migräne weiter verifiziert werden.

\subsection{Limitationen}

Ein Grund für die inhomogenen Ergebnissen in den gesamten Studien mit tDCS und TMS könnte auch sein, dass aktuell nicht genau geklärt ist, welche Regionen im Gehirn bei welcher Form von Migräne über- oder unteraktiviert sind. Möglicherweise liegt bei episodischer Migräne im Gegensatz zur chronischen Migräne eine unterschiedliche Aktivierung des Gehirnes vor (Magis 2015). So gibt es Hinweise, dass bei episodischer Migräne das kortikale Präaktivierungsniveau herabgesetzt sein könnte bzw. zwischen den Anfällen wechselt (Magis et al. 2013), während bei chronischer Migräne dauerhaft ein erhöhtes Aktivierungsniveau vorzuliegen scheint (wie eine nicht endende Migräneattacke) (Magis et al. 2013; Magis 2015). Aufgrund dessen ist es möglich, dass der therapeutische Effekt von Verfahren zur Modulierung der Hirnaktivität nicht generalisierbar, sondern abhängig von der aktuellen kortikalen Aktivierung ist und wahrscheinlich nicht jeder Migränepatient gleich gut mit dem selben Verfahren behandelt werden kann (Magis et al. 2013). Eine dynamische Dysfunktion des visuellen Kortex kann auch im Falle unserer Studie den Behandlungserfolg gemindert haben. Möglicherweise ist es in Zukunft sinnvoll, zunächst mit TMS zu überprüfen, ob aktuell eine inhibitorische oder aktivierende Stimulation durchzuführen ist. Dabei ist zu beachten, dass es aufgrund der Dynamik schwierig ist, ein Ausgangsniveau zu verifizieren. In unserer Studie wurden sowohl Patienten mit episodischer Migräne als auch chronischer Migräne eingeschlossen. Möglicherweise ist es jedoch ratsam, diese beiden Patientenkollektive aufgrund der oben genannten Hinweise auf verschiedene Präaktivierungsniveaus zu trennen. 
Eventuell war in unserer Studie die Intensität, Häufigkeit und/oder Dauer der Stimulation nicht stark und lang genug, um die Frequenz der Migräneattacken zu senken. Verglichen mit vorangegangenen Studien, welche anodale tDCS über dem primär motorischen Kortex für die Behandlung von chronischen Schmerzpatienten verwendeten, ist die gewählte Intensität unserer Stimulation ein wenig geringer. Fregni et al. stimulierten Patienten mit chronischen Rückenschmerzen mit einer Intensität von $2 \mathrm{~mA}$ an fünf aufeinander folgenden Tagen und beobachteten damit ein Nachlassen des Schmerzes von 58\% nach Stimulation (Fregni et al. 2006b).

Antal et al. wiederum verwendeten nur $1 \mathrm{~mA}$-Intensität, aber eine kleinere Stimulationselektrode $\left(16 \mathrm{~cm}^{2}\right)$ in einer heterogenen Gruppe von Schmerzpatienten und erreichten damit eine durchschnittliche Schmerzreduktion von 38\% (Antal et al. 2010).

Eine geringere tDCS-Intensität ist für die Verblindung hingegen von Vorteil. Wie bei Furubayashi et al. berichtet wird, ist die Verwendung von $3 \mathrm{~mA}$ bereits relativ schmerzhaft (Furubayashi et al. 2008). Demzufolge ist die Chance, dass die Patienten die Placebo- von der Verumstimulation unterschieden können, bei dem Gebrauch von $1 \mathrm{~mA}$ deutlich geringer als bei der Verwendung von $2 \mathrm{~mA}$.

Da in Hinsicht auf die Häufigkeit des Auftretens von Begleitphänomenen durch tDCS Jucken, Kribbeln und Brennen keine Unterschiede von den Patienten nach Placebo- bzw. Verumstimulation angegeben wurden und Müdigkeit sogar in einem deutlich höheren Prozentsatz nach Placebostimulation empfunden wurde, scheinen die Patienten die Unterschiede zwischen Placebo- und Verumstimulation nicht wahrgenommen zu haben. Dies weist darauf hin, dass wir in der Studie sehr gute Placebokonditionen geschaffen haben. In zukünftigen Studien sollte dennoch eine gesteigerte Stimulationsdauer und/ oder Frequenz in Betracht gezogen werden.

Eine Limitation in unserer Studie ist, dass die meisten unserer Patienten eine alternative, nicht-pharmakologische Behandlung favorisierten und hieraus möglicherweise eine sehr positive Einstellung gegenüber der tDCS-Behandlung resultierte. Demzufolge kann nicht entschieden werden, ob die beobachteten Verbesserungen durch tDCS nicht auch teilweise auf einen Placeboeffekt zurückzuführen sind. Allerdings trifft dies mutmaßlich auch auf viele andere Patientengruppen der verschiedenen Studien, in denen transkranielle Stimulationsmethoden verwendet werden, zu.

\subsection{Nebenwirkungen und Patientensicherheit}

Die höhere Inzidenz von Kopfschmerzen und Müdigkeit während und nach tDCS in der Placebogruppe ist überraschend und wirft die Frage auf, ob kathodale Stimulation bei Migränepatienten Müdigkeit verringert.

Im Vergleich zu gesunden Probanden (7,8\%) gaben die Migräneprobanden (55,6\%) in einer vorausgegangen Studie gehäuft Kopfschmerzen nach tDCS an (Poreisz et al. 2007). Zudem 
beschrieben Patienten mit chronischen Schmerzen in einer weiteren Studie mit 35-39\% Kopfschmerzen nach tDCS (Antal et al. 2010).

Gleichwohl wurden unsererseits keine ernsthaften Komplikationen wie Krampfanfälle in Verbindung mit tDCS eruiert. Auch wenn die tDCS bereits in der klinischen Anwendung in vielen Phase-II-Studien getestet wurde, so ist die Auswirkung von aufeinander folgenden Sitzungen kortikaler Stimulationen bei verschiedenen Patientenpopulationen noch nicht vollständig bekannt. 


\section{$5 \quad$ Zusammenfassung}

Mit tDCS steht ein nebenwirkungsarmes Verfahren zur Verfügung, welches die kortikale Erregbarkeit verändern kann. Ausgehend von der Annahme, dass die Entstehung von Migräne mit einer erhöhte neuronale Erregbarkeit des visuellen Kortex assoziiert ist, stellt kathodale tDCS des okzipitalen Kortex durch Absenken der kortikalen Erregbarkeit möglicherweise eine wirksame Migräneprophylaxe dar.

Die Zielsetzung unserer Studie war es zu überprüfen, ob kathodale tDCS über V1 eine Reduktion der Frequenz, Dauer und Intensität von Migräneattacken bewirken kann.

An der Studie nahmen 30 Patienten teil, von denen 26 in die finale Analyse eingeschlossen werden konnten. Zunächst erhielten alle Patienten über drei Wochen eine Placebostimulation. Während der nachfolgenden drei Wochen erhielten 13 Patienten eine tDCS von $1 \mathrm{~mA}$ über dem okzipitalen Kortex, während die anderen 13 Patienten weiterhin nur eine Placebostimulation erhielten. Die Stimulation war über den gesamten Zeitraum für alle Patienten verblindet.

Zwei Monate vor der Stimulation, während der Stimulation und zwei Monate nach der Stimulation führten die Patienten ein Migränetagebuch, in welchem die Häufigkeit und Dauer der Attacken, die Schmerzintensität und Migräne-assoziierten Tage sowie Begleiterscheinungen dokumentiert wurden.

Zusammenfassend kann die Aussage gemacht werden, dass die kathodale Stimulation über V1 eine effektive Wirkung in der Migräneprophylaxe bezogen auf die Dauer der Migräneattacke und durchschnittliche Schmerzintensität im Vergleich zur Baseline aufweist. Aufgrund dessen hoffen wir, dass die von uns erhobenen Daten zu weiteren Forschungsarbeiten mit größeren Patientengruppen anregen, um unsere Ergebnisse zu validieren. Zudem wäre es bei größeren Patientengruppen auch möglich, einen statistischen Vergleich der Patienten mit MA und MO durchzuführen, um zu klären, ob die beiden Gruppen eine voneinander abweichende neuronale Funktionalität aufweisen. 


\section{$6 \quad$ Anhang}

\subsection{Verwendete Fragebögen}

\begin{tabular}{|c|c|}
\hline $\begin{array}{c}\text { UNIVERSITÄTSMEDIZIN } \\
\text { GÖTTINGEN }\end{array}$ & \\
Zentrum Neurologische Medizin, \\
Abt. Klinische Neurophysiologie \\
Robert-Koch-Str. 40, 37075 Göttingen \\
Direktor: Prof. Dr. med. Walter Paulus \\
Tel.: 0551/396650
\end{tabular}

1. Geschlecht:

\begin{tabular}{|l|}
$\square$ Männlich \\
\hline$\square$ Weiblich \\
\hline
\end{tabular}

2. Alter:

3. An wie vielen Untersuchungen haben Sie teilgenommen?

4. Haben Sie an diesen Untersuchungen als gesunde/r Proband/in $\square$ oder als Patient/in

$\square$ teilgenommen?

Wegen welcher Krankheit wurde eine Untersuchung mit der Stimulation an Ihnen durchgeführt?

5. Über welcher Region wurden Sie stimuliert?

$\square$ motorische Hirnrinde (eine Elektrode über dem li. Schädel und die andere über der re. Augenbraue oder umgekehrt)

$\square$ Sehrinde (eine Elektrode am Hinterkopf und die andere über der Mitte des Schädels)

$\square$ parietale Hirnrinde (eine Elektrode hinter dem Ohr und die andere über der Mitte des Schädels)

$\square$ frontale Hirnrinde (eine Elektrode über dem li. vorderen Schädel und die andere über der re. Augenbraue oder umgekehrt)

6. Haben Sie bei Beginn und/oder Ende der Stimulation einen Lichtblitz wahrgenommen?

\begin{tabular}{|l|l|}
\hline$\square$ ja & $\square$ nein \\
\hline
\end{tabular}

7. Haben Sie während der Stimulation unter den Elektroden / der Spule Schmerz gefühlt?

\begin{tabular}{|l|l|}
\hline ja & $\square$ nein \\
\hline
\end{tabular}

Wenn ja, wie stark?

$\square$-gering $\square$-mäßig $\square$-mittelmäßig $\quad \square$-stark $\square$-nicht aushaltbar

8. Hat ihre Kopfhaut während der Stimulation unter den Elektroden / der Spule gekribbelt? 


\begin{tabular}{|l|l}
\hline ja & $\square$ nein
\end{tabular}

Wenn ja, wie stark?

$\square$-gering $\square$-mäßig $\square$-mittelmäßig $\square$-stark $\square$-nicht aushaltbar

9. Hat ihre Kopfhaut während der Stimulation unter den Elektroden / der Spule gejuckt?

\begin{tabular}{|l|l|}
\hline$\square$ ja & $\square$ nein \\
\hline
\end{tabular}

Wenn ja, wie stark?

$\square$-gering $\square$-mäßig $\square$-mittelmäßig $\quad \square$-stark $\square$-nicht aushaltbar

10. Hat ihre Kopfhaut während der Stimulation unter den Elektroden / der Spule gebrannt?

\begin{tabular}{|l|l|}
\hline$\square$ ja & $\square$ nein \\
\hline
\end{tabular}

Wenn ja, wie stark?

$\square$-gering $\square$-mäßig $\square$-mittelmäßig $\quad \square$-stark $\square$-nicht aushaltbar

11. Waren Sie während der Stimulation müde?

\begin{tabular}{|l|l|}
\hline$\square$ ja & $\square$ nein \\
\hline
\end{tabular}

Wenn ja, wie müde waren Sie?

$\square$-gering $\square$-mäßig $\square$-mittelmäßig $\square$-stark $\square$-äußert

12. Waren Sie während der Stimulation nervös?

\begin{tabular}{|l|l|}
\hline$\square$ ja & $\square$ nein \\
\hline
\end{tabular}

Wenn ja, wie nervös waren Sie?

$\square$-gering $\square$-mäßig $\square$-mittelmäßig $\square$-stark $\square$-äußert

13. Haben Sie während der Stimulation Konzentrationsprobleme gehabt?

\begin{tabular}{|l|l|}
\hline ja & $\square$ nein \\
\hline
\end{tabular}

Wenn ja, wie starke Konzentrationsprobleme haben Sie bemerkt?

$\square$-gering $\square$-mäßig $\square$-mittelmäßig $\square$-stark $\square$-äußert

14. Haben Sie während der Stimulation Sehprobleme gehabt?

\begin{tabular}{|l|l|}
\hline$\square$ ja & $\square$ nein \\
\hline
\end{tabular}

Wenn ja, wie starke Sehprobleme haben Sie bemerkt?
$\square$-gering
$\square$-mäßig
$\square$-mittelmäßig
$\square$-stark
-äußert

15. Haben Sie während der Stimulation Kopfschmerzen gehabt?

\begin{tabular}{|l|l|}
\hline$\square$ ja & $\square$ nein \\
\hline
\end{tabular}


Wenn ja, wie starke Kopfschmerzen Sie gefühlt haben?

$\square$-gering $\square$-mäßig $\square$-mittelmäßig $\square$-stark $\square$-äußert

16. Haben Sie während der Stimulation etwas ungewöhnliches gefühlt?

\begin{tabular}{|l|l|}
\hline$\square$ ja & $\square$ nein \\
\hline
\end{tabular}

17. Haben Sie während der Stimulation noch etwas anderes gefühlt?

\begin{tabular}{|l|l|}
\hline$\square$ ja & $\square$ nein \\
\hline
\end{tabular}

Wenn ja, bitte beschreiben Sie:

18. War die Stimulation für Sie unangenehm?

\begin{tabular}{|l|l|}
\hline$\square$ ja & $\square$ nein \\
\hline
\end{tabular}

Wenn ja, wie sehr?

$\square$-gering $\square$-mäßig $\square$-mittelmäßig $\square$-stark $\square$-äußert

19. Haben Sie nach der Stimulation unter den Elektroden / der Spule Schmerz gefühlt?

\begin{tabular}{|l|l|}
\hline$\square$ ja & $\square$ nein \\
\hline
\end{tabular}

Wenn ja, wie stark?

$\square$-gering $\square$-mäßig $\square$-mittelmäßig $\quad \square$-stark $\square$-nicht aushaltbar

20. Hat ihre Kopfhaut nach der Stimulation unter den Elektroden / der Spule gekribbelt?

\begin{tabular}{|l|l|}
\hline$\square$ ja & $\square$ nein \\
\hline
\end{tabular}

Wenn ja, wie stark?

$\square$-gering $\square$-mäßig $\square$-mittelmäßig $\quad \square$-stark $\square$-nicht aushaltbar

21. Hat ihre Kopfhaut nach der Stimulation unter den Elektroden / der Spule gejuckt?

\begin{tabular}{|l|l|}
\hline ja & $\square$ nein \\
\hline
\end{tabular}

Wenn ja, wie stark?

$\square$-gering $\square$-mäßig $\square$-mittelmäßig $\quad \square$-stark $\square$-nicht aushaltbar

22. Hat ihre Kopfhaut nach der Stimulation unter den Elektroden / der Spule gebrannt?

\begin{tabular}{|l|l|}
\hline$\square$ ja & $\square$ nein \\
\hline
\end{tabular}

Wenn ja, wie stark?

$\square$-gering $\square$-mäßig $\square$-mittelmäßig $\quad \square$-stark $\square$-nicht aushaltbar

23. Waren Sie nach der Stimulation müde?

\begin{tabular}{|l|l|}
\hline$\square$ ja & $\square$ nein \\
\hline
\end{tabular}


Wenn ja, wie müde waren Sie?

$\square$-gering $\square$-mäßig $\square$-mittelmäßig $\square$-stark $\square$-äußert

24. Waren Sie nach der Stimulation nervös?

\begin{tabular}{|l|l|}
\hline$\square$ ja & $\square$ nein \\
\hline
\end{tabular}

Wenn ja, wie nervös waren Sie?

$\square$-gering $\square$-mäßig $\square$-mittelmäßig $\square$-stark $\square$-äußert

25. Haben Sie nach der Stimulation Konzentrationsprobleme gehabt?

\begin{tabular}{|l|l|}
\hline$\square$ ja & $\square$ nein \\
\hline
\end{tabular}

Wenn ja, wie starke Konzentrationsprobleme haben Sie bemerkt?

$\square$-gering $\square$-mäßig $\square$-mittelmäßig $\square$-stark $\square$-äußert

26. Haben Sie nach der Stimulation Sehprobleme gehabt?

\begin{tabular}{|l|l|}
\hline$\square$ ja & $\square$ nein \\
\hline
\end{tabular}

Wenn ja, wie starke Sehprobleme haben Sie bemerkt?

$\square$-gering $\square$-mäßig $\square$-mittelmäßig $\square$-stark $\square$-äußert

27. Haben Sie nach der Stimulation Kopfschmerzen gehabt?

\begin{tabular}{|l|l|}
\hline ja & $\square$ nein \\
\hline
\end{tabular}

Wenn ja, wie starke Kopfschmerzen Sie gefühlt haben?

$\square$-gering $\square$-mäßig $\square$-mittelmäßig $\square$-stark $\square$-äußert

28. War Ihnen nach der Stimulation übel?

\begin{tabular}{|l|l|}
\hline$\square$ ja & $\square$ nein \\
\hline
\end{tabular}

Wenn ja, wie lange war Ihnen übel (in Stunden)?

29. Haben Sie nach der Stimulation erbrochen?

\begin{tabular}{|l|l|}
\hline$\square$ ja & $\square$ nein \\
\hline
\end{tabular}

Wenn ja, wie oft? 
30. Haben Sie nach der Stimulation Schlafstörungen gehabt?

\begin{tabular}{|l|l}
$\square$ ja & $\square$ nein
\end{tabular}

Wenn ja, wie viele Tage lang?

31. Haben Sie nach der Stimulation Manie-Symptom (Aufregung) bemerkt?

\begin{tabular}{|l|l|}
\hline$\square$ ja & $\square$ nein \\
\hline
\end{tabular}

Wenn ja, wie lange hat das gedauert (in Stunden)?

32. War Ihnen nach der Stimulation kalt?

\begin{tabular}{|l|l|}
\hline$\square$ ja & $\square$ nein \\
\hline
\end{tabular}

Wenn ja, wie lange hat das gedauert (in Stunden)?

33. War Ihnen nach der Stimulation warm?

\begin{tabular}{|l|l|}
\hline 口ja & $\square$ nein \\
\hline
\end{tabular}

Wenn ja, wie lange hat das gedauert (in Stunden)?

3

34. Haben Sie nach der Stimulation etwas ungewöhnliches gefühlt?

\begin{tabular}{|l|l|}
\hline$\square \mathrm{ja}$ & $\square$ nein \\
\hline
\end{tabular}

35. Haben Sie nach der Stimulation noch etwas anderes gefühlt?

\begin{tabular}{|l|l|}
\hline$\square$ ja & $\square$ nein \\
\hline
\end{tabular}

Wenn ja, bitte beschreiben Sie:

36. Haben Sie einen Unterschied zwischen den Stimulationssorten bemerkt?

\begin{tabular}{|l|l|}
\hline$\square$ ja & $\square$ nein \\
\hline
\end{tabular}

Wenn ja, bitte beschreiben Sie das:

37. Haben Sie Angst vor der Stimulation gehabt?

\begin{tabular}{|l|l|}
\hline ja & $\square$ nein \\
\hline
\end{tabular}

38. Möchten Sie später erneut eine Untersuchung mit der Stimulation mitmachen?

$\square$ ja $\square$ nein

Bitte überprüfen Sie, ob Sie alle Fragen beantwortet haben.

Vielen Dank 


\section{Migräne Fragebogen}

Name: Bitte hier ausfüllen

Datum: Bitte hier ausfüllen

Geschlecht:

\begin{tabular}{|l|}
$\square$ Männlich \\
\hline$\square$ Weiblich \\
\hline
\end{tabular}

Alter: nummer

Telefonnummer: nummer

1. Leiden Sie länger als 6 Monate an Kopfschmerzen?

\begin{tabular}{|l|l}
$\square$ ja & $\square$ nein
\end{tabular}

Seit wann? Bitte hier ausfüllen

2. Haben Sie mehr als 10 Kopfschmerzattacken pro Jahr?

\begin{tabular}{|l|l|}
\hline ja & $\square$ nein \\
\hline
\end{tabular}

Wie viele pro Jahr? Bitte hier ausfüllen

3. Dauern gewöhnlich Ihre Kopfschmerzanfälle ohne Einnahme von Medikamenten zwischen 4-72 Stunden?

\begin{tabular}{|l|l|}
\hline ja & $\square$ nein \\
\hline
\end{tabular}

Wie lange dauert ungefähr ein Kopfschmerzanfall (in Stunden)? Bitte hier ausfüllen

4. Treten die Schmerzen einseitig auf?

\begin{tabular}{|l|l|}
\hline$\square$ ja & $\square$ nein \\
\hline
\end{tabular}

5. Beeinträchtigen die Schmerzanfälle oft stark Ihr Freizeitleben?

\begin{tabular}{|l|l|}
\hline$\square$ ja & $\square$ nein \\
\hline
\end{tabular}

Wie oft pro Monat? Bitte hier ausfüllen

6. Behindern Sie die Schmerzanfälle oft bei der Ausübung Ihrer beruflichen Tätigkeit?

\begin{tabular}{|l|l|}
\hline ja & $\square$ nein \\
\hline
\end{tabular}

Wie oft pro Monat? Bitte hier ausfüllen

7. Empfinden Sie den Schmerz oft als klopfend oder hämmernd?

\begin{tabular}{|l|l|}
\hline ja & $\square$ nein \\
\hline
\end{tabular}

8. Empfinden Sie Ihre Kopfschmerzen als dumpf und drückend?

\begin{tabular}{|l|l|}
\hline ja & $\square$ nein \\
\hline
\end{tabular}


9. Treten Ihre Kopfschmerzen beidseitig auf?

$\square$ ji a nein

10. Dauern Ihre Kopfschmerzanfälle 30 Min. bis 7 Tagen?

\begin{tabular}{|l|l|}
\hline ja & $\square$ nein \\
\hline
\end{tabular}

Wie lange dauert ein Kopfschmerzanfall? Bitte hier ausfüllen

11. Treten Ihre Kopfschmerzen anfallsweise auch täglich auf?

\begin{tabular}{|l|l|}
\hline ja & $\square$ nein \\
\hline
\end{tabular}

12. Leiden Sie während Ihrer Kopfschmerzanfälle an Übelkeit und/oder Erbrechen?

\begin{tabular}{|l|l|}
\hline ja & $\square$ nein \\
\hline
\end{tabular}

13. Gehen den Schmerzattacken oftmals Müdigkeit oder Stimmungsschwankungen voraus? \begin{tabular}{|l|l|}
\hline ja & $\square$ nein \\
\hline
\end{tabular}

14. Leiden Sie gewöhnlich während Ihrer Schmerzattacken unter Übelkeit und/oder Erbrechen, sowie unter Licht-, Lärmempfindlichkeit?

\begin{tabular}{|l|l|}
$\square$ ja & $\square$ nein \\
\hline
\end{tabular}

15. Verstärkt sich die Schmerzintensität durch Aktivität?

\begin{tabular}{|l|l|}
\hline ja & $\square$ nein \\
\hline
\end{tabular}

16. Sehen Sie manchmal vor Ihren Schmerzanfallen Lichtblitze oder Zickzacklinien?

\begin{tabular}{|l|l|}
\hline ja & $\square$ nein \\
\hline
\end{tabular}

17. Sehen Sie manchmal verschwommen vor Ihren Schmerzanfällen?

\begin{tabular}{|l|l|}
\hline ja & $\square$ nein \\
\hline
\end{tabular}

18. Haben Sie manchmal Lähmungserscheinungen, Missempfindungen oder Sprachstörungen vor Thren Anfällen?

\begin{tabular}{|l|l|}
$\square$ ja & $\square$ nein \\
\hline
\end{tabular}

19. Haben Sie manchmal Taubheitsgefühle im Bereich der Lippen oder Hände vor Ihren Schmerzanfällen?

\begin{tabular}{|l|l|}
\hline ja & $\square$ nein \\
\hline
\end{tabular}

20. Leiden Sie derzeit noch unter anderen Erkrankungen?

\begin{tabular}{|l|l|}
\hline ja & $\square$ nein \\
\hline
\end{tabular}

Wenn Ja, welche und seit wann? Bitte hier ausfüllen

21. Haben Sie jemals an einer schweren chronischen Erkrankung gelitten?

\begin{tabular}{|l|l|}
\hline ja & $\square$ nein \\
\hline
\end{tabular}

Wenn Ja, welche und wann? (z. B.: Epilepsie, Herzkrankheiten, psychiatrische Erkrankungen...)Bitte hier ausfüllen 
22. Nehmen Sie irgendwelche Medikamente ein?

\begin{tabular}{|l|l|}
\hline$\square$ ja & $\square$ nein \\
\hline
\end{tabular}

Wenn Ja, welche, wie viel und wie häufig? (z. B.: Pille, Schmerztabletten, Schlaftabletten...) Bitte hier ausfüllen

23. Rauchen Sie mehr als 10 Zigaretten pro Tag?

\begin{tabular}{|l|l|}
\hline$\square$ ja & $\square$ nein \\
\hline
\end{tabular}

Zigaretten pro Tag? Bitte hier ausfüllen

24. Trinken Sie regelmäßig Alkohol oder konsumieren Sie Drogen?

\begin{tabular}{|l|l|}
\hline ja & $\square$ nein \\
\hline
\end{tabular}

25. Hat jemand in der Familie Migräne?

\begin{tabular}{|l|l|}
\hline ja & $\square$ nein \\
\hline
\end{tabular}

Wer hat Migräne? Bitte hier ausfüllen

26. Nur für Frauen Sind Sie in den Wechseljahren?

\begin{tabular}{|l|l|}
\hline ja & $\square$ nein \\
\hline
\end{tabular}

Bitte überprïfen Sie, ob Sie alle Fragen beantwortet haben.

Vielen Dank! 


\subsection{Verwendetes Migränetagebuch}

Kopfschmerztagebuch mit Legende:

Monat:

Name:

\begin{tabular}{|c|c|c|c|c|c|c|c|c|c|}
\hline Datum & Schweregrad & $\begin{array}{l}\text { Dauer in } \\
\text { Stunden }\end{array}$ & Begleitsymptome & Gemütszustand & $\begin{array}{l}\text { Schmerzbekämpfungsmaß- } \\
\text { nahmen }\end{array}$ & \begin{tabular}{|l|} 
Erfolg \\
nach Stun- \\
den
\end{tabular} & $\begin{array}{l}\text { Anhalten des } \\
\text { Erfolgs in } \\
\text { Stunden }\end{array}$ & $\begin{array}{l}\text { Schweregrad nach Wir- } \\
\text { kungseintritt der Maß- } \\
\text { nahme }\end{array}$ & $\begin{array}{l}\text { Mögliche } \\
\text { Auslöser } \\
\text { (Trigger) }\end{array}$ \\
\hline \multicolumn{10}{|l|}{1} \\
\hline \multicolumn{10}{|l|}{2} \\
\hline \multicolumn{10}{|l|}{$\cdots$} \\
\hline \multicolumn{10}{|l|}{31} \\
\hline \multicolumn{2}{|c|}{$\begin{array}{l}\text { Schweregrad Scala: } \\
\text { 0 - keine Schmerzen } \\
1 \text { - leichte Schmerzen die normale All- } \\
\text { tagsaktivität erlauben } \\
\text { 2-Mäsige Schmerzen die Alltagsakti- } \\
\text { vität stören aber nicht verhindern. } \\
\text { 3- Schwere Kopfschmerzen die nor- } \\
\text { male Alltagsaktivität unmog̈lich ma- } \\
\text { chen, Bettruhe kann erforderlich sein }\end{array}$} & \multicolumn{3}{|c|}{ 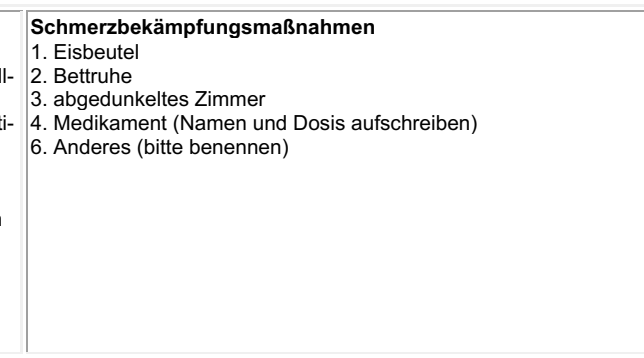 } & \multicolumn{2}{|c|}{$\begin{array}{l}\text { Mögliche Auslöser (Trigger) } \\
\text { 1. Alkohol } \\
\text { 2. Schokolade, Kaffee } \\
\text { 3. Alter reifer Käse } \\
\text { 4. Citrusfrüchte } \\
\text { 5. Glutamat, chinesisches Essen } \\
\text { 6. Hunger } \\
\text { 7. Nüsse } \\
\text { 8. Zwiebel, salziges Essen } \\
\text { 9. Stress } \\
\text { 10. Müdigkeit, zu viel oder zu wenig Schlaf, Wechsel des } \\
\text { Schlaf- Wachrhythmus } \\
\text { 11. Medikamente vergessen (was) } \\
\text { 12. Angestrengtes Sehen, andere optische Reize }\end{array}$} & & & \\
\hline \multicolumn{2}{|c|}{$\begin{array}{l}\text { Frauen markieren die Tage ihrer } \\
\text { Menstruation. }\end{array}$} & \multicolumn{3}{|c|}{$\begin{array}{l}\text { Begleitsymptome } \\
\text { 1. Lichtscheu } \\
\text { 2. Lärmempenfindlichkeit } \\
\text { 3. Übelkeit } \\
\text { 4. Erbrechen } \\
\text { 5. andere (benennen) }\end{array}$} & \multicolumn{2}{|l|}{$\begin{array}{l}\text { Gemütszustand } \\
\text { 1. Fit zum Bäume ausreißen } \\
\text { 2. etwas nervös } \\
\text { 3. bedrückt, } \\
\text { 4. energielos, müde } \\
\text { 5. hoffnungslos, hiflos }\end{array}$} & & & \\
\hline
\end{tabular}




\section{$7 \quad$ Literaturverzeichnis}

Aderjan D: Zentrale Verarbeitung repetitiver trigeminonozizeptiver Reize bei Patienten mit Migräne. Med. Diss. Hamburg. 2012

Afra J, Mascia A, Gérard P, Maertens de Noordhout A, Schoenen J (1998a): Interictal cortical excitability in migraine: a study using transcranial magnetic stimulation of motor and visual cortices. Ann Neurol 44, 209-215

Afra J, Cecchini AP, De Pasqua V, Albert A, Schoenen J (1998b): Visual evoked potentials during long periods of pattern-reversal stimulation in migraine. Brain A J Neurol 121, 233-241

Amassian VE, Cracco RQ, Maccabee PJ, Cracco JB, Rudell AP, Eberle L (1998): Transcranial magnetic stimulation in study of the visual pathway. J Clin Neurophysiol 15, 288-304

Andrasik F (2004): Behavioral treatment of migraine: current status and future directions. Expert Rev Neurother 4, 403-413

Andrasik F (2010): Biofeedback in headache: an overview of approaches and evidence. Cleve Clin J Med 77 (Suppl 3), 72-76

Antal A, Kincses TZ, Nitsche MA, Bartfai O, Paulus W (2004): Excitability changes induced in the human primary visual cortex by transcranial direct current stimulation: direct electrophysiological evidence. Invest Ophthalmol Vis Sci $\underline{45}, 702-707$

Antal A, Temme J, Nitsche MA, Varga ET, Lang N, Paulus W (2005): Altered motion perception in migraineurs: evidence for interictal cortical hyperexcitability. Cephalalgia an Int J headache $\underline{25}, 788-794$

Antal A, Arlt S, Nitsche MA, Chadaide Z, Paulus W (2006): Higher variability of phosphene thresholds in migraineurs than in controls: a consecutive transcranial magnetic stimulation study. Cephalalgia $\underline{26}, 865-870$

Antal A, Lang N, Boros K, Nitsche MA, Siebner HR, Paulus W (2008): Homeostatic metaplasticity of the motor cortex is altered during headache-free intervals in migraine with aura. Cereb Cortex 18, 2701-2705

Antal A, Terney D, Kühnl S, Paulus W (2010): Anodal transcranial direct current stimulation of the motor cortex ameliorates chronic pain and reduces short intracortical inhibition. J Pain Symptom Manage 39, 890-903

Antal A, Paulus W, Nitsche MA (2011): Electrical stimulation and visual network plasticity. Restor Neurol Neurosci $\underline{29}$, 365-374

Antal A, Alekseichuk I, Bikson M, Brockmöller J, Brunoni AR, Chen R, Cohen LG, Dowthwaite G, Ellrich J, Flöel A, et al. (2017): Low intensity transcranial electric stimulation: Safety, ethical, legal regulatory and application guidelines. Clin Neurophysiol 128, 1774-1809 
Anttila V, Stefansson H, Kallela M, Todt U, Terwindt GM, Calafato MS, Nyholt DR, Dimas AS, Freilinger T, Müller-Myhsok B, et al. (2010): Genome-wide association study of migraine implicates a common susceptibility variant on 8q22.1. Nat Genet $\underline{42}, 869-873$

Anttila V, Winsvold BS, Gormley P, Kurth T, Bettella F, McMahon G, Kallela M, Malik R, De Vries B, Terwindt G, et al. (2013): Genome-wide meta-analysis identifies new susceptibility loci for migraine. Nat Genet 45, 912-917

Aurora SK, Ahmad BK, Welch KM, Bhardhwaj P, Ramadan NM (1998): Transcranial magnetic stimulation confirms hyperexcitability of occipital cortex in migraine. Neurology $\underline{50}, 1111-$ 1114

Aurora SK, Welch KM (2000): Migraine: imaging the aura. Curr Opin Neurol 13, 273-276

Auvichayapat P, Janyacharoen T, Rotenberg A, Tiamkao S, Krisanaprakornkit T, Sinawat S, Punjaruk W, Thinkhamrop B, Auvichayapat N (2012): Migraine prophylaxis by anodal transcranial direct current stimulation, a randomized, placebo-controlled trial. J Med Assoc Thai 95, 1003-1012

Ayata C, Jin H, Kudo C, Dalkara T, Moskowitz MA (2006): Suppression of cortical spreading depression in migraine prophylaxis. Ann Neurol $\underline{59}, 652-661$

Bahra A, Matharu, Buchel C, Frackowiak R, Goadsby PJ (2001): Brainstem activation specific to migraine headache. Lancet 357, 1016-1017

Bhola R, Kinsella E, Giffin N, Lipscombe S, Ahmed F, Weatherall M, Goadsby PJ (2015): Singlepulse transcranial magnetic stimulation (sTMS) for the acute treatment of migraine: evaluation of outcome data for the UK post market pilot program. J Headache Pain $\underline{16}, 535$

Bikson M, Datta A, Rahman A, Scaturro J (2010): Electrode montages for tDCS and weak transcranial electrical stimulation: role of "return" electrode's position and size. Clin Neurophysiol 121, 1976-1978

Bikson M, Grossman P, Thomas C, Zannou AL, Jiang J, Adnan T, Mourdoukoutas AP, Kronberg G, Truong D, Boggio P, et al. (2016): Safety of Transcranial Direct Current Stimulation: Evidence Based Update 2016. Brain Stimul 2, 641-661

Bindman LJ, Lippold OCJ, Redfearn JWT (1964): The action of brief polarizing currents on the cerebral cortex of the rat (1) during currrent flow and (2) in the production of long-lasting after effects. J Physiol 172, 369-382

Bohotin V, Fumal A, Vandenheede M, Bohotin C, Schoenen J (2003): Excitability of visual V1-V2 and motor cortices to single transcranial magnetic stimuli in migraine: a reappraisal using a figure-of-eight coil. Cephalalgia $\underline{23}$, 264-270

Bolay H, Reuter U, Dunn AK, Huang Z, Boas DA, Moskowitz MA (2002): Intrinsic brain activity triggers trigeminal meningeal afferents in a migraine model. Nat Med $\underline{8}, 136-142$

Bolognini N, Spandri V, Ferraro F, Salmaggi A, Molinari ACL, Fregni F, Maravita A (2015): Immediate and Sustained Effects of 5-Day Transcranial Direct Current Stimulation of the Motor Cortex in Phantom Limb Pain. J Pain 16, 657-665 
Bolzoni F, Pettersson LG, Jankowska E (2013a): Evidence for long-lasting subcortical facilitation by transcranial direct current stimulation in the cat. J Physiol 591, 3381-3399

Bolzoni F, Bączyk M, Jankowska E (2013b): Subcortical effects of transcranial direct current stimulation in the rat. J Physiol $\underline{591}, 4027-4042$

Boros K, Poreisz C, Paulus W, Antal A (2009): Does the menstrual cycle influence the motor and phosphene thresholds in migraine? Eur J Neurol 16, 367-374

Bowyer SM, Aurora SK, Moran JE, Tepley N, Welch KMA (2001): Magnetoencephalographic fields from patients with spontaneous and induced migraine aura. Ann Neurol $\underline{50}, 582-587$

Brandes JL, Saper JR, Diamond M, Couch JR, Lewis DW, Schmitt J, Neto W, Schwabe S, Jacobs D (2004): Topiramate for migraine prevention: a randomized controlled trial. JAMA 291, 965973

Brennan KC, Bates EA, Shapiro RE, Zyuzin J, Hallows WC, Huang Y, Lee HY, Jones CR, Fu YH, Charles AC, Ptáček LJ (2013): Casein kinase io mutations in familial migraine and advanced sleep phase. Sci Transl Med $\underline{5}, 183$ ra56

Breslau N, Rasmussen BK (2001): The impact of migraine: Epidemiology, risk factors, and comorbidities. Neurology $\underline{56}, 4-12$

Brighina F, Piazza A, Daniele O, Fierro B (2002): Modulation of visual cortical excitability in migraine with aura: effects of $1 \mathrm{~Hz}$ repetitive transcranial magnetic stimulation. Exp Brain Res $\underline{145}, 177-181$

Brighina F, Piazza A, Vitello G, Aloisio A, Palermo A, Daniele O, Fierro B (2004): rTMS of the prefrontal cortex in the treatment of chronic migraine: a pilot study. J Neurol Sci 227, 67-71

Brighina F, Palermo A, Aloisio A, Francolini M, Giglia G, Fierro B (2006): Levetiracetam in the prophylaxis of migraine with aura: a 6-month open-label study. Clin Neuropharmacol 29, 338 342

Brighina F, Palermo A, Fierro B (2009): Cortical inhibition and habituation to evoked potentials: Relevance for pathophysiology of migraine. J Headache Pain 10, 77-84

Brigo F, Storti M, Nardone R, Fiaschi A, Bongiovanni LG, Tezzon F, Manganotti P (2012): Transcranial magnetic stimulation of visual cortex in migraine patients: a systematic review with meta-analysis. J Headache Pain $\underline{13}$, 339-349

Brigo F, Storti M, Tezzon F, Manganotti P, Nardone R (2013): Primary visual cortex excitability in migraine: A systematic review with meta-analysis. Neurol Sci $\underline{34}, 819-830$

Brunoni AR, Amadera J, Berbel B, Volz MS, Rizzerio BG, Fregni F (2011): A systematic review on reporting and assessment of adverse effects associated with transcranial direct current stimulation. Int J Neuropsychopharmacol 14, 1133-1145

Bureš J, Burešová $\mathrm{O}$, Křvánek J: The mechanism and application of Leão’s spreading depression of electroencephalographic activity. Academia, Prague 1974

Busch V, Gaul C (2008): Exercise in migraine therapy--is there any evidence for efficacy? A critical review. Headache $\underline{48}, 890-899$ 
Caekebeke JF, Ferrari MD, Zwetsloot CP, Jansen J, Saxena PR (1992):Antimigraine drug sumatriptan increases blood flow velocity in large cerebral arteries during migraine attacks. Neurology $\underline{42}, 1522-1526$

Cao Y, Welch KMA, Aurora SK, Vikingstad EM (1999): Functional MRI-BOLD of visually triggered headache in patients with migraine. Arch Neurol $\underline{56}, 548-554$

Chadaide Z, Arlt S, Antal A, Nitsche MA, Lang N, Paulus W (2007): Transcranial direct current stimulation reveals inhibitory deficiency in migraine. Cephalalgia 27, 833-839

Charles A, Flippen C, Romero Reyes M, Brennan KC (2007): Memantine for prevention of migraine: a retrospective study of 60 cases. J Headache Pain $\underline{8}, 248-250$

Chronicle E, Mulleners W (2004): Anticonvulsant drugs for migraine prophylaxis. Cochrane database Syst Rev $\underline{3}$, CD003226

Chronicle EP, Pearson AJ, Mulleners WM (2006): Objective assessment of cortical excitability in migraine with and without aura. Cephalalgia 26, 801-808

Clarke BM, Upton ARM, Kamath MV, Al-Harbi T, Castellanos CM (2006): Transcranial magnetic stimulation for migraine: clinical effects. J Headache Pain $\mathbf{7}, 341-346$

Conforto AB, Amaro E Jr, Goncalves AL, Mercante JP, Guendler VZ, Ferreira JR, Kirschner CC, Peres MF (2014): Randomized, proof-of-principle clinical trial of active transcranial magnetic stimulation in chronic migraine. Cephalalgia $\underline{34}, 464-472$

Coppola G, Pierelli F, Schoenen J (2007): Is the cerebral cortex hyperexcitable or hyperresponsive in migraine? Cephalalgia 27, 1429-1439

Costa C, Tozzi A, Rainero I, Cupini LM, Calabresi P, Ayata C, Sarchielli P (2013): Cortical spreading depression as a target for anti-migraine agents. J Headache Pain 14, 62

Costain R, Redfearn JWT, Lippold OCJ (1964): A controlled trial of the therapeutic effect of polarization of the brain in depressive illness. Br J Psychiatry 110, 786-799

Creutzfeldt OD, Fromm GH, Kapp H (1962): Influence of transcortical d-c currents on cortical neuronal activity. Exp Neurol $\underline{5}, 436-452$

Cutrer FM, Sorensen AG, Weisskoff RM, Ostergaard L, Sanchez Del Rio M, Lee EJ, Rosen BR, Moskowitz MA (1998): Perfusion-weighted imaging defects during spontaneous migrainous aura. Ann Neurol 43, 25-31

Dasilva AF, Mendonca ME, Zaghi S, Lopes M, Dossantos MF, Spierings EL, Bajwa Z, Datta A, Bikson M, Fregni F (2012): tDCS-induced analgesia and electrical fields in pain-related neural networks in chronic migraine. Headache $\underline{52}, 1283-1295$

De Fusco M, Marconi R, Silvestri L, Atorino L, Rampoldi L, Morgante L, Ballabio A, Aridon P, Casari G (2003): Haploinsufficiency of ATP1A2 encoding the Na+/K+ pump alpha2 subunit associated with familial hemiplegic migraine type 2. Nat Genet $\underline{33}$, 192-196

Deutsche Gesellschaft für Neurologie (2012): Therapie der Migräne (Leitlinien für Diagnostik und Therapie in der Neurologie). S1- Leitlinie der Deutschen Gesellschaft für Neurologie. http://www.awmf.org/leitlinien/detail/11/030-057.h; Zugriff am 10.12.2015 
Dichgans M, Herzog J, Freilinger T, Wilke M, Auer DP (2005): 1H-MRS alterations in the cerebellum of patients with familial hemiplegic migraine type 1. Neurology $\underline{64}, 608-613$

Diener HC: Migräne. Ein Buch mit sieben Siegeln? 100 Fragen und 100 Antworten. 3. überarb. Auflage; Georg Thieme Verlag, Stuttgart New York 2002

Diener HC, Limmroth V (1994): The treatment of migraine. Rev Contemp Pharmacother $\underline{5}, 271-$ 284

Diener HC, Leniger T: Migräne und Komorbidität. In: Diener HC (Hrsg.): Kopfschmerzen. Georg Thieme Verlag, Stuttgart New York 2003, 58-59

Diener HC, Matias-Guiu J, Hartung E, Pfaffenrath V, Ludin HP, Nappi G, De Beukelaar F (2002): Efficacy and tolerability in migraine prophylaxis of flunarizine in reduced doses: a comparison with propranolol $160 \mathrm{mg}$ daily. Cephalalgia 22, 209-221

Diener HC, Tfelt-Hansen P, Dahlöf C, Láinez MJA, Sandrini G, Wang SJ, Neto W, Vijapurkar U, Doyle A, Jacobs D (2004): Topiramate in migraine prophylaxis--results from a placebocontrolled trial with propranolol as an active control. J Neurol 251, 943-950

Diener HC, Dodick DW, Aurora SK, Turkel CC, DeGryse RE, Lipton RB, Silberstein SD, Brin MF (2010): OnabotulinumtoxinA for treatment of chronic migraine: results from the doubleblind, randomized, placebo-controlled phase of the PREEMPT 2 trial. Cephalalgia $\underline{30}, 804-814$

Duckrow RB (1991): Regional cerebral blood flow during spreading cortical depression in conscious rats. J Cereb blood flow Metab Off J Int Soc Cereb Blood Flow Metab 11, 150-154

Dundas JE, Thickbroom GW, Mastaglia FL (2007): Perception of comfort during transcranial DC stimulation: Effect of $\mathrm{NaCl}$ solution concentration applied to sponge electrodes. Clin Neurophysiol 118, 1166-1170

Ebersberger A (2002): Pathophysiology of migraine: models to explain the generation of migraine headache. Anaesthesist $\underline{51}, 661-667$

Edvinsson L, Goadsby PJ (1994): Neuropeptides in migraine and cluster headache. Cephalalgia an Int $\mathrm{J}$ headache 14, 320-327

Eikermann-Haerter K, Ayata C (2010): Cortical spreading depression and migraine. Curr Neurol Neurosci Rep 10, 167-173

Ekbom K (1975): Alprenolol for migraine prophylaxis. Headache 15, 129-132

Ekbom K, Zetterman M (1977): Oxprenolol in the treatment of migraine. Acta Neurol Scand $\underline{56}$, $181-184$

Ensink FBM: Vom Mutterkorn zum selektiven Serotoninrezeptorantagonisten - Historische Aspekte der Entwicklung eines spezifischen Migränetherapeutikums. In: Ensink FBM, Soyaka D (Hrsg.): Migräne - aktuelle Aspekte eines altbekannten Leidens. Springer Verlag, Berlin Heidelberg 1994, 25-60

Epstein CM, Lah JJ, Meador K, Weissman JD, Gaitan LE, Dihenia B (1996): Optimum stimulus parameters for lateralized suppression of speech with magnetic brain stimulation. Neurology 47, 1590-1593 
Evers S, Rahmann A, Vollmer-Haase J, Husstedt IW (2002): Treatment of headache with botulinum toxin A--a review according to evidence-based medicine criteria. Cephalalgia 22, 699-710

Evers S, May A, Fritsche G, Kropp P, Lampl C, Limmroth V, Malzacher V, Sandor P, Straube A, Diener HC (2008): Akuttherapie und Prophylaxe der Migräne, Leitlinie der Deutschen Migräne- und Kopfschmerzgesellschaft und der Deutschen Gesellschaft für Neurologie. Nervenheilkunde 27, 933-949

Fagerlund AJ, Hansen OA, Aslaksen PM (2015): Transcranial direct current stimulation as a treatment for patients with fibromyalgia: A randomized controlled trial. Pain $\underline{156}$, 62-71

Ferrari MD, Haan J, Palotie A: Genetics of migraine. In: Olesen J, Goadsby PJ, Ramadan NM, Tfelt- Hansen P, Welch KMA (Hrsg.): The Headaches. 3. Auflage; Lippincott Williams\&Wilkins, Philadelphia 2006, 251-259

Fertonani a., Pirulli C, Miniussi C (2011): Random Noise Stimulation Improves Neuroplasticity in Perceptual Learning. J Neurosci $\underline{31}, 15416-15423$

Fertonani A, Ferrari C, Miniussi C (2015): What do you feel if I apply transcranial electric stimulation? Safety, sensations and secondary induced effects. Clin Neurophysiol $\underline{126}, 2181-$ 2188

Feuerstein T: Antikonvulsiva, Konvulsiva - Pharmarkotherapie der Epilepsie. In: Aktories K, Förstermann U, Hofmann F, Starke K (Hrsg.): Allgemeine und spezielle Pharmakologie und Toxikologie. 11. Auflage; Urban \& Fischer Verlag/Elsevier, München 2013, 269-279

Feuerstein T, Diener HC: Pharmakologie des Serotonins - Pharmakotherapie primärer Kopfschmerzen. In: Aktories K, Förstermann U, Hofmann F, Starke K (Hrsg.): Allgemeine und spezielle Pharmakologie und Toxikologie. 11. Auflage; Urban \& Fischer Verlag/Elsevier, München 2013, 191-198

Forssman B, Lindblad CJ, Zbornikova V (1983): Atenolol for migraine prophylaxis. Headache 2 33, $188-190$

Fregni F, Gimenes R, Valle AC, Ferreira MJL, Rocha RR, Natalle L, Bravo R, Rigonatti SP, Freedman SD, Nitsche MA, et al. (2006a): A randomized, sham-controlled, proof of principle study of transcranial direct current stimulation for the treatment of pain in fibromyalgia. Arthritis Rheum 54, 3988-3998

Fregni F, Boggio PS, Lima MC, Ferreira MJL, Wagner T, Rigonatti SP, Castro AW, Souza DR, Riberto M, Freedman SD, et al. (2006b): A sham-controlled, phase II trial of transcranial direct current stimulation for the treatment of central pain in traumatic spinal cord injury. Pain $\underline{122}$, 197-209

Freitag FG, Collins SD, Carlson HA, Goldstein J, Saper J, Silberstein S, Mathew N, Winner PK, Deaton R, Sommerville K (2002): A randomized trial of divalproex sodium extended-release tablets in migraine prophylaxis. Neurology $\underline{58}, 1652-1659$

Friberg L, Olesen J, Iversen HK, Sperling B (1991): Migraine pain associated with middle cerebral artery dilatation: reversal by sumatriptan. Lancet $\underline{338}, 13-17$ 
Furubayashi T, Terao Y, Arai N, Okabe S, Mochizuki H, Hanajima R, Hamada M, Yugeta A, Inomata-Terada S, Ugawa Y (2008): Short and long duration transcranial direct current stimulation (tDCS) over the human hand motor area. Exp Brain Res 185, 279-286

Gartside IB (1968): Mechanisms of sustained increases of firing rate of neurons in the rat cerebral cortex after polarization: reverberating circuits or modification of synaptic conductance? Nature 220, 382-383

Gawel M, Connolly JF, Rose FC (1983): Migraine patients exhibit abnormalities in the visual evoked potential. Headache $\underline{23}, 49-52$

Gervil M, Ulrich V, Kyvik KO, Olesen J, Russell MB (1999): Migraine without aura: a populationbased twin study. Ann Neurol 46, 606-611

Gerwig M, Niehaus L, Kastrup O, Stude P, Diener HC (2005): Visual cortex excitability in migraine evaluated by single and paired magnetic stimuli. Headache $\underline{45}$, 1394-1399

Gleixner C, Müller M, Wirth S: Migräne. In: Müller M (Hrsg.): Neurologie und Psychatrie. 6. Auflage; Medizinische Verlags- und Informationsdienste, Breisach 2007/08, 96-99

Goadsby PJ, Edvinsson L (1993): The trigeminovascular system and migraine: studies characterizing cerebrovascular and neuropeptide changes seen in humans and cats. Ann Neurol $\underline{33}, 48-56$

Goadsby PJ, Sprenger T (2010): Current practice and future directions in the prevention and acute management of migraine. Lancet Neurol $\underline{9}, 285-298$

Göbel H: Die Kopfschmerzen: Ursachen, Mechanismen, Diagnostik und Therapie in der Praxis. 2. Auflage; Springer Verlag, Berlin Heidelberg 2004

Göbel H: Migräne: Diagnostik - Therapie - Prävention. Springer Verlag, Berlin Heidelberg 2012

Grafstein B: Neuronal release of potassium during spreading depression. In: Brazier MAB (Hrsg.): Brain function: cortical excitability and steady potentials; relations of basic research to space biology. Volume 1; University of California Press, Berkeley Los Angeles 1963, 87-124

Gunaydin S, Soysal A, Atay T, Arpaci B (2006): Motor and occipital cortex excitability in migraine patients. Can J Neurol Sci $\underline{33}, 63-67$

Gursoy-Ozdemir Y, Qiu J, Matsuoka N, Bolay H, Bermpohl D, Jin H, Wang X, Rosenberg GA, Lo EH, Moskowitz MA (2004): Cortical spreading depression activates and upregulates MMP-9. J Clin Invest 113, 1447-1455

Haas DC, Kent PF, Friedman DI (1993): Headache caused by a single lesion of multiple sclerosis in the periaqueductal gray area. Headache $\underline{33}, 452-455$

Hadjikhani N, Sanchez Del Rio M, Wu O, Schwartz D, Bakker D, Fischl B, Kwong KK, Cutrer FM, Rosen BR, Tootell RBH, et al. (2001): Mechanisms of migraine aura revealed by functional MRI in human visual cortex. Proc Natl Acad Sci U S A 98, 4687-4692

Hagner M: Das proteushafte Antlitz der Migräne - Überlegungen zur Kultur eines altbekannten Leidens. In: Ensink FBM, Soyaka D (Hrsg.): Migräne - aktuelle Aspekte eines altbekannten Leidens. Springer Verlag, Berlin Heidelberg 1994, 1-24 
Hallett M (2000): Transcranial magnetic stimulation and the human brain. Nature 406, 147-150

Hallett M (2007): Transcranial magnetic stimulation: a primer. Neuron $\underline{55}, 187-199$

Hans M, Luvisetto S, Williams ME, Spagnolo M, Urrutia A, Tottene A, Brust PF, Johnson EC, Harpold MM, Stauderman KA, Pietrobon D (1999): Functional consequences of mutations in the human alpha1A calcium channel subunit linked to familial hemiplegic migraine. J Neurosci $\underline{19}, 1610-1619$

Hattori Y, Moriwaki A, Hori Y (1990): Biphasic effects of polarizing current on adenosine-sensitive generation of cyclic AMP in rat cerebral cortex. Neurosci Lett 116, 320-324

Herzog A: Migräne und das serotonerge System. Med. Diss. Würzburg 2008

Holroyd KA, Penzien DB, Cordingley GE (1991): Propranolol in the management of recurrent migraine: a meta-analytic review. Headache $\underline{31}, 333-340$

Huang J, Cooper TG, Satana B, Kaufman DI, Cao Y (2003): Visual distortion provoked by a stimulus in migraine associated with hyperneuronal activity. Headache $\underline{43}$, 664-671

Hucklenbroich P, Evers S: Medizingeschichte des Schmerzes am Beispiel der Migräne. In: Gralow I, Husstedt IW, Bothe HW, Evers S, Hürter A, Schilgen M (Hrsg.): Schmerztherapie interdisziplinär: Pathophysiologie, Diagnostik, Therapie. Schattauer, Stuttgart 2002, 12-15

Islam N, Aftabuddin M, Moriwaki A, Hattori Y, Hori Y (1995): Increase in the calcium level following anodal polarization in the rat brain. Brain Res $\underline{684}$, 206-208

Iyer MB, Mattu U, Grafman J, Lomarev M, Sato S, Wassermann EM (2005): Safety and cognitive effect of frontal DC brain polarization in healthy individuals. Neurology $\underline{64}, 872-875$

Johannsson V, Nilsson LR, Widelius T, Jäverfalk T, Hellman P, Akesson JA, Olerud B, Gustafsson CL, Raak A, Sandahl G (1987): Atenolol in migraine prophylaxis a double-blind cross-over multicentre study. Headache 27, 372-374

Jonas A: Entwicklung der Migräne und der „Contingent Negative Veration“ (CNV) bei Kindern mit Migräne - ein Fellow-up über 8 Jahre. Med. Diss. Kiel 2012

Judit A, Sándor PS, Schoenen J (2000): Habituation of visual and intensity dependence of auditory evoked cortical potentials tends to normalize just before and during the migraine attack. Cephalalgia 20, 714-719

Jürgens T, Paulus W, Tronnier V, Gaul C, Lampl C, Gantenbein A, May A, Diener HC (2011): Neuromodulierende Verfahren bei primären Kopfschmerzen. Nervenheilkunde $\underline{30}$, 47-58

Kahlig KM, Rhodes TH, Pusch M, Freilinger T, Pereira-Monteiro JM, Ferrari MD, Van Den Maagdenberg AMJM, Dichgans M, George AL (2008): Divergent sodium channel defects in familial hemiplegic migraine. Proc Natl Acad Sci U S A 105, 9799-9804

Kahlil N: Investigation of visual function in migraine using visual evoked potentials and visual psychophysiological tests. Phil. Diss. London 1991

Kalita J, Laskar S, Bhoi SK, Misra UK (2016): Efficacy of single versus three sessions of high rate repetitive transcranial magnetic stimulation in chronic migraine and tension-type headache. J Neurol 263, 2238-2246 
Kaube H, Hoskin KL, Goadsby PJ (1992): Activation of the trigeminovascular system by mechanical distension of the superior sagittal sinus in the cat. Cephalalgia an Int J headache $\underline{12}, 133-136$

Kaube H, Keay KA, Hoskin KL, Bandler R, Goadsby PJ (1993): Expression of c-Fos-like immunoreactivity in the caudal medulla and upper cervical spinal cord following stimulation of the superior sagittal sinus in the cat. Brain Res $\underline{629}$, 95-102

Keidel M: Ursachen, Formen, Therapie. Verlag C. H. Beck, München 2007

Kelman L (2007): The triggers or precipitants of the acute migraine attack. Cephalalgia $\underline{27}$, 394-402

Klapper J (1997): Divalproex sodium in migraine prophylaxis: a dose-controlled study. Cephalalgia $\underline{17}, 103-108$

Knight YE, Goadsby PJ (2001): The periaqueductal grey matter modulates trigeminovascular input: A role in migraine? Neuroscience 106, 793-800

Kotzian M: Wirkung von Spreading Depression auf die intrazelluläre Aktivität von Neuronen im Hirnschnitt der Maus. Med. Diss. Münster 2009

Kow LM, van Harreveld A (1972): Ion and water movements in isolated chicken retinas during spreading depression. Neurobiology $2,61-69$

Kraft A, Roehmel J, Olma MC, Schmidt S, Irlbacher K, Brandt SA (2010): Transcranial direct current stimulation affects visual perception measured by threshold perimetry. Exp Brain Res 207, 283-290

Kraig RP, Nicholson C (1978): Extracellular ionic variations during spreading depression. Neuroscience $\underline{3}, 1045-1059$

Krishnan C, Santos L, Peterson MD, Ehinger M (2015): Safety of noninvasive brain stimulation in children and adolescents. Brain Stimul $\underline{8}, 76-87$

Kropp P, Göbel H, Dworschak M, Heinze A (1996): Elektrophysiologische Untersuchungen bei Kopfschmerzen: Die "contingent negative variation" (CNV). Schmerz 10, 130-134

Láinez MJA, Freitag FG, Pfeil J, Ascher S, Olson WH, Schwalen S (2007): Time course of adverse events most commonly associated with topiramate for migraine prevention. Eur J Neurol $\underline{14}$, 900-906

Lampl C, Katsarava Z, Diener HC, Limmroth V (2005): Lamotrigine reduces migraine aura and migraine attacks in patients with migraine with aura. J Neurol Neurosurg Psychiatry $\underline{76}, 1730$ 1732

Lauritzen M (1994): Pathophysiology of the migraine aura. The spreading depression theory. Brain 117, 199-210

Liebetanz D (2002): Pharmacological approach to the mechanisms of transcranial DC-stimulationinduced after-effects of human motor cortex excitability. Brain $\underline{125}$, 2238-2247

Liebetanz D, Fregni F, Monte-Silva KK, Oliveira MB, Amâncio-dos-Santos A, Nitsche M, Guedes RC (2006): After-effects of transcranial direct current stimulation (tDCS) on cortical spreading depression. Neurosci Lett $\underline{398}, 85-90$ 
Limmroth V, Diener HC (2003a): Kopfschmerzen: Neues zu Pathophysiologie und Therapie der Migräne. Notfall Medizin 29, 32-41

Limmroth V, Diener HC: Pathophysiologie, Genetik und Klinik der Migräne. In: Diener HC (Hrsg.): Kopfschmerzen. Georg Thieme Verlag, Stuttgart New York 2003b, 35-40

Limmroth V, May A, Auerbach P, Wosnitza G, Eppe T, Diener HC (1996): Changes in cerebral blood flow velocity after treatment with sumatriptan or placebo and implications for the pathophysiology of migraine. J Neurol Sci $\underline{138}, 60-65$

Linde K, Rossnagel K (2004): Propranolol for migraine prophylaxis. Cochrane Database Syst Rev 2, CD003225

Linde K, Allais G, Brinkhaus B, Manheimer E, Vickers A, White AR (2009): Acupuncture for migraine prophylaxis. Cochrane Database Syst Rev 1 , CD001218

Lipton RB, Pearlman SH (2010): Transcranial magnetic simulation in the treatment of migraine. Neurotherapeutics $\underline{7}, 204-212$

Lipton RB, Dodick DW, Silberstein SD, Saper JR, Aurora SK, Pearlman SH, Fischell RE, Ruppel PL, Goadsby PJ (2010): Single-pulse transcranial magnetic stimulation for acute treatment of migraine with aura: a randomised, double-blind, parallel-group, sham-controlled trial. Lancet Neurol $\underline{9}, 373-380$

Louis P (1981): A double-blind placebo-controlled prophylactic study of flunarizine (Sibelium) in migraine. Headache 21, 235-239

Lüllmann H, Mohr K, Wehling M: Pharmakologie und Toxikologie. Arzneimittelwirkungen verstehen - Medikamente gezielt einsetzen. 15. Auflage; Georg Thieme Verlag, Stuttgart New York 2002

Machado S, Arias-Carrión O, Paes F, Vieira RT, Caixeta L, Novaes F, Marinho T, Almada LF, Silva AC, Nardi AE (2013): Repetitive transcranial magnetic stimulation for clinical applications in neurological and psychiatric disorders: an overview. Eurasian J Med 4ㄷ, 191-206

Maeda F, Keenan JP, Tormos JM, Topka H, Pascual-Leone A (2000): Modulation of corticospinal excitability by repetitive transcranial magnetic stimulation. Clin Neurophysiol 111, 800-805

Maertens de Noordhout A, Timsit-Berthier M, Timsit M, Schoenen J (1986): Contingent negative variation in headache. Ann Neurol 19, 78-80

Magis D (2015): Neuromodulation in migraine: state of the art and perspectives. Expert Rev Med Devices 12, 329-339

Magis D, Viganò A, Sava S, d'Elia TS, Schoenen J, Coppola G (2013): Pearls and pitfalls: electrophysiology for primary headaches. Cephalalgia $\underline{33}, 526-539$

Marlow NM, Bonilha HS, Short EB (2013): Efficacy of transcranial direct current stimulation and repetitive transcranial magnetic stimulation for treating fibromyalgia syndrome: a systematic review. Pain Pract $\underline{13}, 131-145$

Mathew NT, Saper JR, Silberstein SD, Rankin L, Markley HG, Solomon S, Rapoport AM, Silber CJ, Deaton RL (1995): Migraine prophylaxis with divalproex. Arch Neurol 52, 281-286 
Merabet LB, Kobayashi M, Barton J, Pascual-Leone A (2003): Suppression of complex visual hallucinatory experiences by occipital transcranial magnetic stimulation: a case report. Neurocase $\underline{9}, 436-440$

Misra UK, Kalita J, Bhoi SK (2012): High frequency repetitive transcranial magnetic stimulation (rTMS) is effective in migraine prophylaxis: an open labeled study. Neurol Res $\underline{34}, 547-551$

Misra UK, Kalita J, Bhoi SK (2013): High-rate repetitive transcranial magnetic stimulation in migraine prophylaxis: A randomized, placebo-controlled study. J Neurol 260, 2793-2801

Mohammad YM, Hughes G, Nkrumah M, Fischell S, Fischell R, Ruppel P, Schweiger J (2006): Self-administered transcranial magnetic stimulation (TMS), during the aura phase, improves and aborts migraine headache. Headache $\underline{46}, 857$

Mohammadianinejad SE, Abbasi V, Sajedi SA, Majdinasab N, Abdollahi F, Hajmanouchehri R, Faraji A (2011): Zonisamide versus topiramate in migraine prophylaxis: a double-blind randomized clinical trial. Clin Neuropharmacol $\underline{34}, 174-177$

Moliadze V, Andreas S, Lyzhko E, Schmanke T, Gurashvili T, Freitag CM, Siniatchkin M (2015): Ten minutes of $1 \mathrm{~mA}$ transcranial direct current stimulation was well tolerated by children and adolescents: Self-reports and resting state EEG analysis. Brain Res Bull 119, 25-33

Mori F, Codecà C, Kusayanagi H, Monteleone F, Buttari F, Fiore S, Bernardi G, Koch G, Centonze D (2010): Effects of anodal transcranial direct current stimulation on chronic neuropathic pain in patients with multiple sclerosis. J Pain 11, 436-442

Moriwaki A (1991): Polarizing currents increase noradrenaline-elicited accumulation of cyclic AMP in rat cerebral cortex. Brain Res $\underline{544}, 248-252$

Moskowitz MA, Bolay H, Dalkara T (2004): Deciphering Migraine Mechanisms: Clues from Familial Hemiplegic Migraine Genotypes. Ann Neurol 55, 276-280

Mottaghy FM, Hungs M, Brugmann M, Sparing R, Boroojerdi B, Foltys H, Huber W, Topper R (1999): Facilitation of picture naming after repetitive transcranial magnetic stimulation. Neurology $\underline{53}, 1806-1806$

Mulkey RM, Herron CE, Malenka RC (1993): An essential role for protein phosphatases in hippocampal long-term depression. Science 261, 1051-1055

Mulleners WM, Chronicle EP, Palmer JE, Koehler PJ, Vredeveld JW (2001a): Suppression of perception in migraine: evidence for reduced inhibition in the visual cortex. Neurology $\underline{56}$, 178-183

Mulleners WM, Chronicle EP, Palmer JE, Koehler PJ, Vredeveld JW (2001b): Visual cortex excitability in migraine with and without aura. Headache $\underline{41}, 565-572$

Nanda RN, Johnson RH, Gray J, Keogh HJ, Melville ID (1978): A double blind trial of acebutolol for migraine prophylaxis. Headache $\underline{18}, 20-22$

Nestoriuc Y, Martin A (2007): Efficacy of biofeedback for migraine: a meta-analysis. Pain $\underline{128}, 111-$ 127 
Nitsche MA, Paulus W (2000): Excitability changes induced in the human motor cortex by weak transcranial direct current stimulation. J Physiol 527, 633-639

Nitsche MA, Paulus W (2001): Sustained excitability elevations induced by transcranial DC motor cortex stimulation in humans. Neurology $\underline{57}, 1899-1901$

Nitsche MA, Paulus W: Transkranielle Gleichstromstimulation. In: Siebner H, Ziemann U (Hrsg.): Das TMS-Buch: Handbuch der transkraniellen Magnetstimulation. Springer Medizin Verlag, Heidelberg 2007, 533-542

Nitsche MA, Nitsche MS, Klein CC, Tergau F, Rothwell JC, Paulus W (2003a): Level of action of cathodal DC polarisation induced inhibition of the human motor cortex. Clin Neurophysiol $114,600-604$

Nitsche MA, Liebetanz D, Antal A, Lang N, Tergau F, Paulus W (2003b): Modulation of cortical excitability by weak direct current stimulation--technical, safety and functional aspects. Suppl Clin Neurophysiol $\underline{56}, 255-276$

Nitsche MA, Liebetanz D, Lang N, Antal A, Tergau F, Paulus W (2003c): Safety criteria for transcranial direct current stimulation (tDCS) in humans. Clin Neurophysiol 114, 2220-2222; author reply 2222-2223

Nitsche MA, Niehaus L, Hoffmann KT, Hengst S, Liebetanz D, Paulus W, Meyer BU (2004a): MRI study of human brain exposed to weak direct current stimulation of the frontal cortex. Clin Neurophysiol 115, 2419-2423

Nitsche MA, Jaussi W, Liebetanz D, Lang N, Tergau F, Paulus W (2004b): Consolidation of human motor cortical neuroplasticity by D-cycloserine. Neuropsychopharmacology $\underline{29}, 1573-1578$

Nitsche MA, Cohen LG, Wassermann EM, Priori A, Lang N, Antal A, Paulus W, Hummel F, Boggio PS, Fregni F, Pascual-Leone A (2008): Transcranial direct current stimulation: State of the art 2008. Brain Stimul 1, 206-223

Olesen J (2006): International Classification of Headache Disorders, Second Edition (ICHD-2): current status and future revisions. Cephalalgia $\underline{26}, 1409-1410$

Olesen J, Friberg L, Olsen TS, Iversen HK, Lassen NA, Andersen AR, Karle A (1990): Timing and topography of cerebral blood flow, aura, and headache during migraine attacks. Ann Neurol 28, $791-798$

Olma MC, Kraft A, Roehmel J, Irlbacher K, Brandt SA (2011): Excitability changes in the visual cortex quantified with signal detection analysis. Restor Neurol Neurosci 29, 453-461

Palm U, Keeser D, Schiller C, Fintescu Z, Reisinger E, Padberg F, Nitsche MA (2008): Skin lesions after treatment with transcranial direct current stimulation (tDCS). Brain Stimul $\underline{1}$, 386-387

Palmer JE, Chronicle EP, Rolan P, Mulleners WM (2000): Cortical hyperexcitability is cortical under-inhibition: Evidence from a novel functional test of migraine patients. Cephalalgia 20, $525-532$

Paulson OB, Newman EA (1987): Does the release of potassium from astrocyte endfeet regulate cerebral blood flow? Science 237, 896-898 
Paulus W (2014): Transkranielle Hirnstimulation: Möglichkeiten und Grenzen. Neuroforum 20 , 202-211

Peeters M, Gunthorpe MJ, Strijbos PJLM, Goldsmith P, Upton N, James MF (2007): Effects of pan- and subtype-selective N-methyl-D-aspartate receptor antagonists on cortical spreading depression in the rat: therapeutic potential for migraine. J Pharmacol Exp Ther 321, 564-572

Peters MAK, Thompson B, Merabet LB, Wu AD, Shams L (2013): Anodal tDCS to V1 blocks

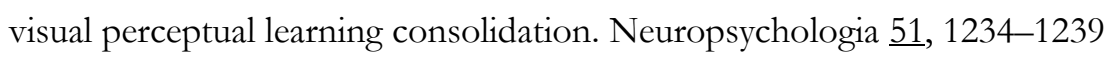

Petri S, Tölle T, Straube A, Pfaffenrath V, Stefenelli U, Ceballos-Baumann A (2009): Botulinum toxin as preventive treatment for migraine: a randomized double-blind study. Eur Neurol $\underline{62}$, 204-211

Pietrobon D, Striessnig J (2003): Neurological diseases: Neurobiology of migraine. Nat Rev Neurosci 4 , 386-398

Pirulli C, Fertonani A, Miniussi C (2013): The role of timing in the induction of neuromodulation in perceptual learning by transcranial electric stimulation. Brain Stimul $\underline{6}, 683-689$

Poreisz C, Boros K, Antal A, Paulus W (2007): Safety aspects of transcranial direct current stimulation concerning healthy subjects and patients. Brain Res Bull $\underline{72}$, 208-214

Purpura D, McMurtry JG (1965): Intracellular activities and evoked potential changes during polarization of motor cortex. J Neurophysiol 28, 166-185

Raskin NH, Hosobuchi Y, Lamb S (1987): Headache may arise from perturbation of brain. Headache 27, 416-420

Rasmussen BK (2001): Epidemiology of headache. Cephalalgia 21, 774-777

Richter F, Lehmenkühler A (2008): Cortical spreading depression (CSD): Ein neurophysiologisches Korrelat der Migräneaura. Schmerz 22, 544-550

Ritter G (1969): Die Neurologie in der hippokratischen Medizin. Nervenarzt 40, 327-333

Rocha S, Melo L, Boudoux C, Foerster Á, Araújo D, Monte-Silva K (2014): Transcranial direct current stimulation in the prophylactic treatment of migraine based on interictal visual cortex excitability abnormalities: A pilot randomized controlled trial. J Neurol Sci $\underline{349}$, 33-39

Rohan JG, Carhuatanta KA, McInturf SM, Miklasevich MK, Jankord R (2015): Modulating Hippocampal Plasticity with In Vivo Brain Stimulation. J Neurosci $\underline{35}$, 12824-12832

Ripoll Rozisky J, da Conceição Antunes L, Brietzke A, de Sousa A, Caumo W: Transcranial direct current stimulation and neuroplasticity. In: Rogers L (Hrsg): Transcranial direct current stimulation (tDCS): Emerging uses, safety and neurobiological effects. Nova Science Publishers, New York NY USA 2015, 59-86

Rüsseler J: Neuropsychologische Therapie: Grundlagen und Praxis der Behandlung kognitiver Störungen bei neurologischen Erkrankungen. Verlag W Kohlhammer, Stuttgart 2009 
Sakrajai P, Janyacharoen T, Jensen MP, Sawanyawisuth K, Auvichayapat N, Tunkamnerdthai O, Keeratitanont K, Auvichayapat P (2014): Pain reduction in myofascial pain syndrome by anodal transcranial direct current stimulation combined with standard treatment: a randomized controlled study. Clin J Pain 무, 1076-1083

Saper JR, Dodick DW, Silberstein SD, McCarville S, Sun M, Goadsby PJ (2011): Occipital nerve stimulation for the treatment of intractable chronic migraine headache: ONSTIM feasibility study. Cephalalgia $\underline{31}, 271-285$

Sasso d'Elia T, Viganò A, Fataki M, Sava SL, Schoenen J, Delphine M (2013): Quadripulse Repetitive Transcranial Magnetic Stimulation on Visual Cortex for Chronic Migraine Prevention: A Pilot-Trial. Cephalgia $\underline{33}, 49$

Schiebler TH: Anatomie. Histologie, Entwicklungsgeschichte, makroskopische und mikroskopische Anatomie, Topographie. 9. vollständig überarb. Auflage; Springer Verlag, Berlin Heidelberg 2005

Schiller F (1975): The migraine tradition. Bull Hist Med 49, 1-19

Schoenen J, Maertens de Noordhout A, Timsit-Berthier M, Timsit M (1986): Contingent negative variation and efficacy of beta-blocking agents in migraine. Cephalalgia $\underline{6}, 229-233$

Schoenen J, Wang W, Albert A, Delwaide PJ (1995): Potentiation instead of habituation characterizes visual evoked potentials in migraine patients between attacks. Eur J Neurol 2 , $115-122$

Schoenen J, Ambrosini A, Sándor PS, Maertens de Noordhout A (2003): Evoked potentials and transcranial magnetic stimulation in migraine: published data and viewpoint on their pathophysiologic significance. Clin Neurophysiol 114, 955-972

Schoenen J, Roberta B, Magis D, Coppola G (2016): Non-invasive neurostimulation methods for migraine therapy : the available evidence. Cephalalgia $\underline{36}, 1170-1180$

Sczesny-Kaiser M, Beckhaus K, Dinse HR, Schwenkreis P, Tegenthoff M, Höffken O (2016): Repetitive Transcranial Direct Current Stimulation Induced Excitability Changes of Primary Visual Cortex and Visual Learning Effects-A Pilot Study. Front Behav Neurosci 10, 116

Seng EK, Holroyd KA (2010): Dynamics of changes in self-efficacy and locus of control expectancies in the behavioral and drug treatment of severe migraine. Ann Behav Med 40, $235-247$

Shepherd AJ (2000): Visual contrast processing in migraine. Cephalalgia 20, 865-880

Shepherd AJ (2001): Increased visual after-effects following pattern adaptation in migraine: a lack of intracortical excitation? Brain 124, 2310-2318

Siebner HR, Ziemann U: Hirnstimulation- Physiologische Grundlagen. In: Siebner HR, Ziemann U (Hrsg.): Das TMS-Buch: Handbuch der transkraniellen Magnetstimulation. Springer Medizin Verlag, Heidelberg 2007, 27-44

Silberstein SD, Neto W, Schmitt J, Jacobs D (2004): Topiramate in migraine prevention: results of a large controlled trial. Arch Neurol 61, 490-495 
Siniatchkin M, Bartsch T: Migräne. In: Siebner HR, Ziemann U (Hrsg.): Das TMS- Buch: Handbuch der transkraniellen Magnetstimulation. Springer Medizin Verlag, Heidelberg 2007, 287-296

Siniatchkin M, Andrasik F, Kropp P, Niederberger U, Strenge H, Averkina N, Lindner V, Stephani U, Gerber WD (2007): Central mechanisms of controlled-release metoprolol in migraine: a double-blind, placebo-controlled study. Cephalalgia 27, 1024-1032

Siniatchkin M, Reich AL, Shepherd AJ, van Baalen A, Siebner HR, Stephani U (2009): Peri-ictal changes of cortical excitability in children suffering from migraine without aura. Pain 147, 132-140

Sjaastad O, Stensrud P (1972): Clinical trial of a beta-receptor blocking agent (LB 46) in migraine prophylaxis. Acta Neurol Scand $\underline{48}, 124-128$

Smith MJ, Keel JC, Greenberg BD, Adams LF, Schmidt PJ, Rubinow DA, Wassermann EM (1999): Menstrual cycle effects on cortical excitability. Neurology $\underline{53}$, 2069-2072

Smith MJ, Adams LF, Schmidt PJ, Rubinow DR, Wassermann EM (2002): Effects of ovarian hormones on human cortical excitability. Ann Neurol $\underline{51}, 599-603$

Sørensen PS, Larsen BH, Rasmussen MJ, Kinge E, Iversen H, Alslev T, Nøhr P, Pedersen KK, Schrøder P, Lademann A (1991): Flunarizine versus metoprolol in migraine prophylaxis: a double-blind, randomized parallel group study of efficacy and tolerability. Headache $\underline{31}, 650-$ 657

Stankewitz A, May A (2007): Cortical excitability and migraine. Cephalalgia 27, 1454-1456

Stankewitz A, May A (2009): The phenomenon of changes in cortical excitability in migraine is not migraine-specific--a unifying thesis. Pain $\underline{145}, 14-17$

Stellar S, Ahrens SP, Meibohm AR, Reines SA (1984): Migraine prevention with timolol. A doubleblind crossover study. JAMA 252, 2576-2580

Stensrud P, Sjaastad O (1980): Comparative trial of Tenormin (atenolol) and Inderal (propranolol) in migraine. Headache 20, 204-207

Stewart WF, Lipton RB, Celentano DD, Reed ML (1992): Prevalence of migraine headache in the United States. Relation to age, income, race, and other sociodemographic factors. JAMA $\underline{267}$, 64-69

Stewart WF, Lipton RB, Liberman J (1996): Variation in migraine prevalence by race. Neurology $\underline{47}, 52-59$

Teepker M, Hötzel J, Timmesfeld N, Reis J, Mylius V, Haag A, Oertel WH, Rosenow F, Schepelmann K (2010): Low-frequency rTMS of the vertex in the prophylactic treatment of migraine. Cephalalgia $\underline{30}, 137-144$

Temme J: Veränderungen der visuellen Wahrnehmung bei Migräne Patienten. Med. Diss. Göttingen 2006 
Tfelt-Hansen P, Standnes B, Kangasneimi P, Hakkarainen H, Olesen J (1984): Timolol vs propranolol vs placebo in common migraine prophylaxis: a double-blind multicenter trial. Acta Neurol Scand $\underline{69}, 1-8$

Trepel M: Neuroanatomie: Struktur und Funktion. 3. Auflage; Urban \& Fischer Verlag/Elsevier GmbH, München 2004

Turi Z, Ambrus GG, Ho KA, Sengupta T, Paulus W, Antal A (2014): When size matters: Large electrodes induce greater stimulation-related cutaneous discomfort than smaller electrodes at equivalent current density. Brain Stimul 7, 460-467

Ulrich V, Gervil M, Kyvik KO, Olesen J, Russell MB (1999): Evidence of a genetic factor in migraine with aura: A population-based Danish twin study. Ann Neurol 45, 242-246

Underwood E (2016): NEUROSCIENCE. Cadaver study challenges brain stimulation methods. Science $\underline{352}, 397$

Vanmolkot KRJ, Babini E, de Vries B, Stam AH, Freilinger T, Terwindt GM, Norris L, Haan J, Frants RR, Ramadan NM, et al. (2007): The novel p.L1649Q mutation in the SCN1A epilepsy gene is associated with familial hemiplegic migraine: genetic and functional studies. Mutation in brief \#957. Online. Hum Mutat 28, 522

van de Ven LL, Franke CL, Koehler PJ (1997): Prophylactic treatment of migraine with bisoprolol: a placebo-controlled study. Cephalalgia 17, 596-599

van Harreveld A (1959): Compounds in brain extracts causing spreading depression of cerebral cortical activity and contraction of crustacean muscle. J Neurochem $\underline{3}, 300-315$

Viganò A, d'Elia TS, Sava SL, Auvé M, De Pasqua V, Colosimo A, Di Piero V, Schoenen J, Magis D (2013): Transcranial Direct Current Stimulation (tDCS) of the visual cortex: a proof-ofconcept study based on interictal electrophysiological abnormalities in migraine. J Headache Pain $\underline{14}, 23$

Wassermann EM, Lisanby SH (2001): Therapeutic application of repetitive transcranial magnetic stimulation: a review. Clin Neurophysiol 112, 1367-1377

Wassermann EM, Zimmermann T (2012): Transcranial magnetic brain stimulation: Therapeutic promises and scientific gaps. Pharmacol Ther $\underline{133}, 98-107$

Weichselbaum A: Die Rolle von Varianten des Kalzium- aktivierten Kaliumkanals KCNN3 bei sporadischer Migräne mit und ohne Aura. Med. Diss. Würzburg. 2004

Weiller C, May A, Limmroth V, Juptner M, Kaube H, Schayck R V, Coenen HH, Diener HC (1995): Brain stem activation in spontaneous human migraine attacks. Nat Med $1,658-660$

Werthmann A, Wieting J: Medizinsche Psychologie und Soziologie, Kapitel 1: Entstehung und Verlauf von Krankheit. In: Emminger H (Hrsg.): Physikum exact: Das gesamte Prüfungswissen für die erste ÄP. 4. Auflage; Georg Thieme Verlag, Stuttgart 2005, 984-1054

Wickmann F, Stephani C, Czesnik D, Klinker F, Timäus C, Chaieb L, Paulus W, Antal A (2015): Prophylactic treatment in menstrual migraine: A proof-of-concept study. J Neurol Sci $\underline{354}$, 103-109 
Wiescher A: Funktionelle retinale Gefässanalyse bei Migränepatienten. Med. Diss. Göttingen 2008

Wilkins A, Nimmo-Smith I, Tait a, McManus C, Della Sala S, Tilley a, Arnold K, Barrie M, Scott S (1984): A neurological basis for visual discomfort. Brain 107, 989-1017

Woods AJ, Antal A, Bikson M, Boggio PS, Brunoni AR, Celnik P, Cohen LG, Fregni F, Herrmann CS, Kappenman ES, et al. (2016): A technical guide to tDCS, and related non-invasive brain stimulation tools. Clin Neurophysiol 127, 1031-1048

Wörz R, Reinhardt-Benmalek B, Föh M, Grotemeyer KH, Scharafinski HW (1992):

Migräneprophylaxe durch Bisoprolol. Ergebnisse einer Doppelblindstudie versus Metoprolol. Fortschr Med 110, 268-272

Wray SH, Mijović-Prelec D, Kosslyn SM (1995): Visual processing in migraineurs. Brain $\underline{118}$, 25-35

Ziemann U: Gepaarte assoziative Stimulation. In: Siebner HR, Ziemann U (Hrsg.): Das TMS-Buch: Handbuch der transkraniellen Magnetstimulation. Springer Medizin Verlag, Heidelberg 2007, $513-522$

Ziemann U: Stimulationstechniken. In: Claßen J, Schnitzler A (Hrsg.): Interventionelle Neurophysiologie : Grundlagen und therapeutische Anwendungen. Georg Thieme Verlag, Stuttgart 2013, 40-51 


\section{Danksagung}

Mein besonderer Dank gilt Frau Professor Dr. Andrea Antal und Herrn Prof. Dr. Walter Paulus für die persönliche Betreuung und vielfältige Unterstützung. Zudem möchte ich dem Team der Klinik für Klinische Neurophysiologie der Universitätsmedizin Göttingen für den stets 